\title{
LA FORMATION ET L'EVOLUTION DE LA BOUCLE GHEZ LE FETUS ET L'AGNEAU KARAKUL. LES FACTEURS DE QUALITÉ DE LA FOURRURE ET LEUR IMPORTANCE POUR LA SÉLECTION
}

PAR

\section{NACER KECHAWARZ}

Avant-propos.

\section{PLAN DU MÉMOIRE}

Introduction.

I. - Matériel et méthodes employés.

A. - Techniques histologiques.

B. - Techniques physiologiques.

C. - Techniques de mesure des caractéristiques des fibres.

II. - Développement et caractéristiques du système pileux.

Historique.

Résultats de recherches.

A. - Modification de la peau au cours de la vie fotale.

B. - Formation et évolution du follicule pileux.

C. - Caractéristiques des fibres chez le fœtus et l'agneau Karakul.

Discussion.

Conclusion.

III. - La boucle de l'agneau Karakul.

Historique.

Résultats de recherches.

PREMIÈRE PARTIE : Bouclage et incurvation des poils.

A. - Bouclage des poils, orientation des follicules et mécanisme de la formation du dessin de la fourrure.

B. - Facteurs d'incurvation et de frisure des poils.

Discussion.

Conclusion.

DEUXIÈME PARTIE : Facteurs liés à la qualité et aux dimensions de la boucle.

Discussion.

Conclusion.

IV. - La qualité de la fourrure.

A. - Le lustre.

B. - Le tannage.

Discussion.

Conclusion. 


\section{Conclusion générale.}

Annexes.

I. - Terminologie zootechnique et commerciale.

II. - Production et commercialisation de la fourrure d'agneau.

III. - La boucle de la toison d'agneau.

IV. - Variétés de la race Karaku1.

\section{AVANT-PROPOS}

Plus de I5 ooo ooo de moutons Karakul paissent à travers les pâturages semi-désertiques de tous les continents à 1'exception de l'Australie. Par sa rusticité, le mouton Karakul arrive, en effet, à s'adapter aux climats très rudes. Aussi, tant par son extension géographique que par son intérêt économique, la race ovine Karakul, appelée communément mouton de Boukhara, occupe une place importante dans l'élevage ovin mondial.

De tout temps, cette race a été réputée pour la fourrure de ses agneaux au beau pelage brillant et bouclé à la naissance. Mais on a souvent tendance à croire que l'élevage de Karakul est exclusivement un élevage de luxe ; en réalité, à côté de la production des peaux d'agneau considérée comme production de luxe, les productions normales qui sont la viande, la laine et le lait, constituent une source non négligeable de revenus.

Ia production de l'astrakan étant la principale activité d'un tiers des éleveurs de notre pays, l'Afghanistan, nous avons entrepris l'étude des causes de la formation de la boucle pendant la vie fœetale et de son effacement après la naissance. Certes, les éleveurs grâce à leur expérience et par de longs et patients travaux de sélection, ont pu améliorer la qualité de la fourrure d'agneau, mais nous espérons que cette contribution aidera à comprendre les phénomènes mis en jeu et par conséquent à les utiliser pour améliorer les conditions de sélection et d'élevage.

Ces travaux n'ont pu être effectués que grâce à des aides nombreuses et diverses qu'il nous est agréable de faire connaître.

Nous avons bénéficié successivement de bourses du Ministère de 1'Agriculture du Royaume d'Afghanistan, de l'Institut national de la Recherche Agronomique, de la Direction des Relations culturelles du ministère des Affaires étrangères, et enfin du Centre national de la Recherche Scientifique.

M. Prenant, professeur d'Histologie et d'Anatomie comparées de la Sorbonne, et M. Thibauli, directeur de la Station de Recherches de Physiologie animale du Centre national de Recherches Zootechniques, nous ont accueilli dans leurs laboratoires, ont dirigé nos travaux et nous ont aidé dans la rédaction de notre mémoire.

M. Rougeot, Chargé de Recherches au Centre national de Recher- 
ches Zootechniques, nous a confié toute sa documentation personnelle sur les fibres des mammifères et il nous a fait bénéficier de son expérience sur la toison des moutons.

Enfin, le troupeau Karakul nous a été fourni par le Centre national de Recherches Zootechniques et les peaux commerciales par MM. LEROLLF et Guiliaumard des Etablissements Chapal.

Que tous trouvent ici l'expression de notre reconnaissance. Je n'aurai garde d'oublier mes camarades des Laboratoires d'Anatomie comparée et de la Station de Physiologie animale, ainsi que le personnel animalier de cette Station, auprès de qui j'ai toujours rencontré une compréhension sympathique et agissante.

\section{INTRODUCTION}

Le mouton domestique "Ovis aries " a été divisé en 4 groupes selon la classification Pallas-Nathusius :

I : Brachyurae (Kurzschwanzschafe).

2: Platyurae (Fettschwanzschafe).

3: Steatopygae (Fettsteiszschafe).

4: Dolychurae (Langschwanzschafe).

Le groupe platyurae, à queue adipeuse est constitué par un grand nombre de races s'étendant de 1'Asie Mineure jusqu'aux Indes. Il existe également mais en moindre densité, en Chine du Nord et en Mandchourie. En Afrique, ce groupe occupe tout le territoire côtier de l'Afrique du Nord, de l'Egypte et de l'Abyssinie. On trouve également des moutons à queue adipeuse en Afrique orientale et méridionale, spécialement dans les zones côtières de ces mêmes territoires, et dans les Iles de Madagascar et de la Réunion (Frolich et Hornitscher, I94I).

Les races ovines à queue adipeuse diffèrent les unes des autres principalement par la forme du corps, la disposition et la forme de la queue adipeuse, les cornes, les oreilles et les caractéristiques de la toison. Elles ont été décrites en I 896 par Sanson, comme des variétés de 1a " Race de Syrie ", dont l'une, la variété persane, produisait de la fourrure que SANSON homologuait à l'astrakan. Ainsi la race Karakul serait originaire des steppes de Palestine et de Syrie. AdameTz (I927), affirme, d'après les explorations de Sendshisli, que des fourrures ressemblant à celles de l'agneau Karakul étaient déjà utilisées en Syrie vers les années I80o avant J. C., pour la garniture des manteaux royaux. Cette origine expliquerait le terme Arabi par lequel on désigne quelquefois la variété noire de Karakul. Puis la race Karakul aurait été exportée vers la Boukharie par les nomades arabes, vers l'an 75I. Mais pour Von HeLmersen (I839) l'Arabi aurait été amené au Turkestan, bien avant la conquête arabe, par des nomades arabiques, tributaires des "Khans" de Boukharie, qui venaient 
du Yémen en parcours annuels, pour faire pâturer leurs troupæaux sur les plateaux dominant 1'Amou Darya. Or, en fait, l'Arabi est une race bien définie, différente du Karakul, sans queue, appartenant au groupe des Steatopygae, et dont les agneaux ont, il est vrai, un pelage légèrement bouclé, C'est donc à la suite d'une erreur d'interprétation que ces auteurs ont situé le berceau de la race Karakul en Syrie ou en Arabie.

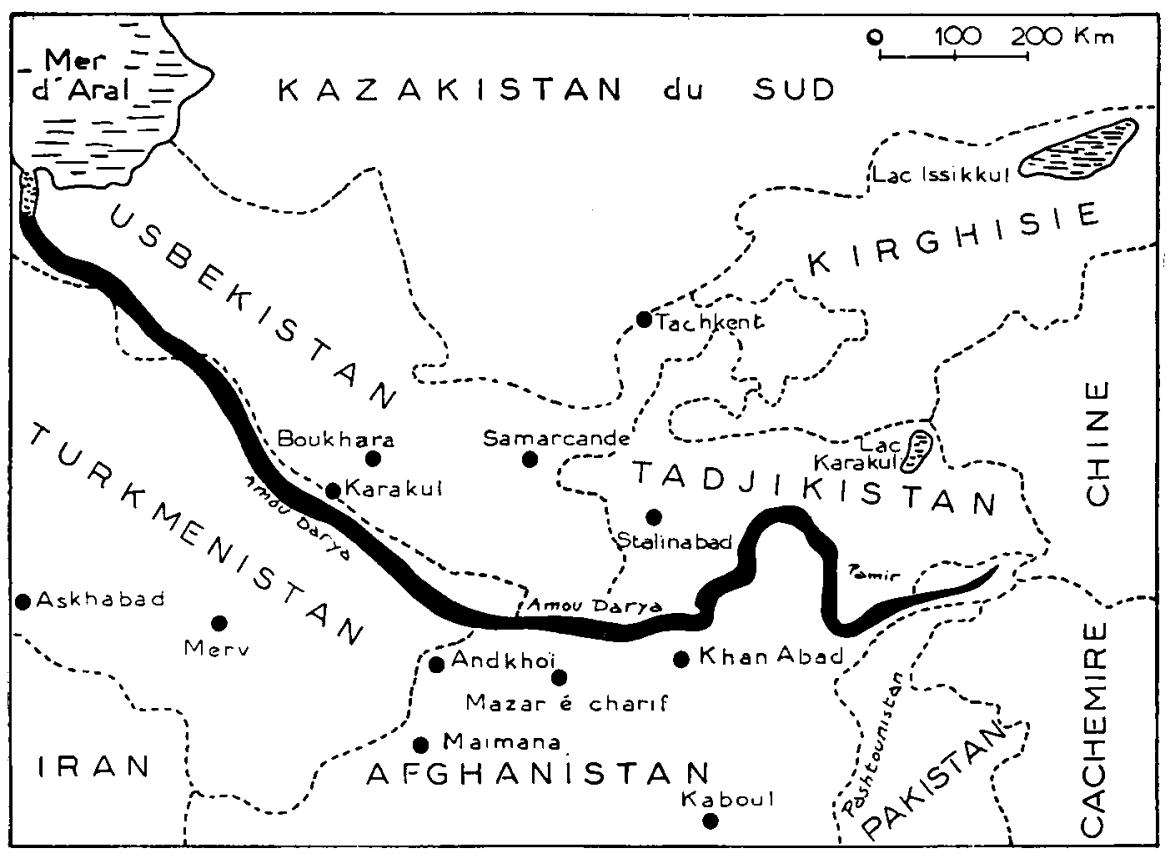

Carte du berceau de la race Karakul

Selon une autre hypothèse, le Karakul serait originaire de la région de 1'Amou-Darya et les fourrures d'agneaux utilisées en Syrie et en Palestine proviendraient d'autres races ovines à queue adipeuse dont les agneaux ont tous un pelage plus ou moins bouclé. C'est d'ailleurs l'opinion généralement avancée. Le terme " Karakul " lui-même est originaire de la région de 1'Amou-Darya et signifie " rose noire ". En effet les boucles de l'agneau Karakul sont appelées fleurs ou roses par les indigènes.

Les travaux d'une mission archéologique américaine ${ }^{(1)}$ ont conduit DUERST (selon RUMEAU, I947) à tirer la conclusion suivante : les fossiles trouvés dans la région d'Anau (Turkestan) ont une ancienneté qui remonte à 7000 ou 8000 ans avant J. C. Les moutons Karakul actuels de la région de Maymen (Turkestan) sont des descendants du mouton fossile d'Anau. Le seul témoignage écrit que nous ayons en ce qui concerne 1'ancienneté

(i) Animal remains from the excavation at Anau by the Pumpelly expedition of the Carnegie Institution. 
de l'exploitation des agneaux Karakul au Turkestan, est celui de IBN Haukar, (année 978) qui écrit : "Les habitants du Turkestan obtiennent parmi leurs agneaux, des agneaux noirs qui, par suite de leur beauté et de leur lustre, se vendent jusqu'à 250 dinars à Khwarizm ".

Exploitée et sélectionnée au voisinage de 1'Amou Darya (Turkestan russe et Turkestan afghan), la race Karakul a lentement été introduite dans le monde entier notamment en Iran, en Irak, en Palestine, en Syrie, en Russie d'Europe, en Turquie, en Allemagne, en Autriche, dans tous les pays d'Europe orientale, en Norvège, dans le Bassin méditerranéen (Italie, Esspagne, France), en Afrique du Nord, en A. O. F., et surtout en Afrique du Sud-Ouest.

Dans ce dernier pays, en I906, le mouton Karakul était inconnu ; il compte aujourd'hui près de 4000 ooo de têtes soit pur, soit croisé, grâce au travail acharné des zootechniciens allemands, pionniers de cette introduction.

Le Karaku1 ne présente pas des aptitudes particulières pour la boucherie au point de vue conformation : cependant la viande est d'une bonne qualité moyenne, plutôt fine.

Sa production laitière n'est pas négligeable avec une moyenne de $67,7 \mathrm{~kg}$ à 8,5 p. Ioo de M. G. en I 40 jours (Iwanov, I933), ce qui permettrait de valoriser le troupeau, les agneaux étant sacrifiés à la naissance.

La toison du mouton Karakul adulte est du type hétérotriche : on y distingue en effet très facilement des poils longs, grossiers et raides à côté des brins de laine plus courts et fins. Ces deux types de fibres se trouvent dans des proportions variables. On peut estimer à $3,8 \mathrm{~kg}$ pour le mâle et $2,5 \mathrm{~kg}$ pour la femelle, la production annuelle par tête, avec un rendement de $58 \mathrm{p}$. Ioo environ.

Cette toison est utilisée pour la confection de tapis, pantoufles, sangles, tissus de sport, d'ameublement, et enfin tissus ou couvertures tyroliens ou écossais. Aussi la toison Karakul compte-t-elle une gamme très étendue d'utilisation et de consommateurs ; son débouché commercial est donc largement assuré.

Les agneaux Karakul naissent avec un pelage bouclé et brillant. Mais à la naissance tous les agneaux ne présentent pas le même état de développement de bouclage. Il y a donc pour chaque agneau une période optimale correspondant au meilleur état de la boucle pendant laquelle il doit être sacrifié. Passé cette période la boucle disparaît, la toison évoluant rapidement vers la toison d'adulte. Le bouclage est donc un phénomène transitoire. Or, c'est lui qui donne à la fourrure toute sa valeur. Il nous a donc paru intéressant d'étudier le mécanisme de la formation et de l'évolution de la boucle. Cette étude a été facilitée en nous référant à des toisons d'autres races étudiées en même temps.

Le problème du lustre a également attiré notre attention car il 
constitue le deuxième élément, après le dessin de l'ensemble des boucles, sur lequel est basée l'appréciation de la fourrure.

On sait que la peau d'agneau n'est utilisée qu'après avoir subi un certain nombre de traitements industriels (confitage, séchage, tannage et teinture). On est donc tenté de connaître la réaction de la peau et de ses boucles vis-à-vis de ces traitements et de savoir quelles sont les modifications subies et comment elles se produisent.

Tels sont les différents problèmes que nous avons étudiés. Dans un chapitre annexe, nous traiterons de la terminologie zootechnique et commerciale, ainsi que des principaux stades de la préparation et de la vente des peaux.

\section{CHAPITRE PREMIER}

\section{MATERIEL ET METHODES UTILISES}

L'étude que nous allons exposer a été effectuée sur :

75 brebis et agneaux Karakul dont 30 purs et 45 croisés ayant un degré de dominance de $3 / 4$ à I5/I6 ;

I6 fœtus Karakul;

6 fœtus Bizet;

6 agneaux et adultes Bizet;

Quelques agneaux et adultes Mérinos, Ile-de-France et Limousine ;

I30 peaux d'agneaux Karakul d'origine afghane, russe, sud-africaine et française.

Nous avons été amenés, pour l'étude de la peau et des fibres chez l'embryon, l'agneau et 1'adulte, à utiliser des techniques histologiques, physiologiques et d'autres techniques d'appréciation et de mesure des fibres. Nous les décrirons successivement pour que le lecteur intéressé par une technique puisse aisément s'y reporter.

\section{A. - TECHNIQUES HISTOLOGIQUES}

\section{Fixation.}

Les échantillons de peaux obtenus par biopsie ou sur les animaux sacrifiés, ont été fixés au formol salé à ro p. Ioo qui permet une conservation de longue durée. D'autres fixateurs tels que le Bouin aqueux, le Bouin Hollande, 1'alcool, ont été utilisés ; cependant, le formol salé a eu notre préférence pour les travaux d'histologie topographique et la recherche des fibres élastiques sous-épidermiques, étant donné la grande quantité du liquide nécessaire et la durée relativement longue de conservation que permet ce fixateur. Pour la recherche du glycogène, nous avons utilisé le Gendre.

On sait que les pièces de peau s'enroulent ou se plissent au cours de 
la fixation, de la déshydratation et de l'inclusion, de sorte qu'il est très difficile d'obtenir des coupes d'une assez grande surface au même niveau des follicules. Nous avons surmonté cette difficulté en plaçant les échantillons entre deux lames de verre et en augmentant la durée des bains. Entre le verre et la peau, nous avons intercalé quelques couches de papier buvard.

\section{2o Eclaircissage et inclusion.}

L'éclaircissage des pièces est obtenu par trois bains de benzène d'une demi-heure chacun.

L'inclusion a été effectuée dans la paraffine à $52^{\circ}$ pour les fœetus jeunes et à $56^{\circ}$ pour les foetus âgés, les agneaux et les adultes. Les résultats obtenus après inclusion dans la paraffine n'ont pas toujours donné satisfaction, certains poils se détachant des follicules au cours des opérations suivantes.

Ceci nous a conduit à faire des inclusions mixtes à la celloïdine paraffine.

Solution à $2 \mathrm{p}$. Ioo de celloïdine dans le benzoate de méthyle : Io-40 jours à la température normale ;

Paraffine : 6-36 heures selon l'épaisseur des pièces.

\section{$3^{\circ}$ Coupe ot coloration.}

Les échantillons ont été coupés à $2,5^{-} 5^{-7,5}$-I0-I5, et $20 \mu$ et colorés sur coupes longitudinales et transversales.

Les colorations utilisées furent les suivantes :

Hemalum acide de Masson-Eosine.

Mallory.

Eosine, Aurentia, Orange G, Bleu de Toluidine.

Safranine-Vert lumière et Safranine picrique.

Bleu polychrome.

Triple coloration de Prenant (Hematoxyline, Eosine, Vert lumière).

Fuchsine-paraldehyde (procédé de GABE, I953).

Schiff selon la réaction de Bauer.

Nous avons fait une large utilisation de la coloration S. A. C. P. I. C. (Safranine, Alcool $\mathrm{IOO}^{\circ}$, Carmin aluné de Grenacher, Picro-Indigo-Carmin), qui donne de bons résultats pour l'histologie de la peau (AUBER, I95I).

\section{$4^{0}$ Structure hemicylindrique des fibres.}

Cette structure a été mise en évidence en employant différents colorants basiques tels que le bleu de méthylène, la safranine, la fuchsine basique et notamment le vert Janus (HoRIo et KonDo, I953) et l'eau de brome après une coloration au bleu de méthylène (LEVEAU, CEBE et PARISOT, I953). 
L,es meilleurs résultats furent obtenus en utilisant le bleu polychrome (Rougeot, I956). Pour la race Karakul, nous avons travaillé sur des fibres blanches des variétés grise et "abrache " ainsi que sur des fibres noires dépigmentées par l'eau oxygénée à 50 volumes.

\section{$5^{\circ}$ Observation par transparence des préparations de peau de forte épaisseur.}

Des lambeaux de peau de I à $300 \mathrm{~cm}^{2}$ de surface ont été rasés après la fixation, déshydratés et éclaircis au benzoate ou mieux encore au salicylate de méthyle pour des examens à la loupe binoculaire. D'autre part, des coupes longitudinales d'une épaisseur d'environ roo $\mu$ et plus, ont été exécutées à main levée. Les poils et les follicules sont ainsi visibles par transparence grâce à leur pigmentation.

Pour mettre en évidence par transparence sous la loupe binoculaire les follicules pileux produisant des poils non pigmentés, nous avons cherché à colorer sélectivement l'appareil folliculaire. Les résultats les plus satisfaisants furent obtenus en fixant les pièces au Bouin, puis en éliminant l'acide picrique en excès par l'eau lithinée qui décolore la peau tout en laissant les fibres teintes en jaune ; après un rinçage à l'eau distillée, on colore les coupes à la safranine puis on différencie à l'alcool absolu jusqu'à la décoloration du tissu conjonctif.

\section{B. - TECHNIQUES PHYSIOLOGIQUES}

\section{1 o Diagnostic de la gestation.}

Avant l'extraction des foetus nous avons cherché à vérifier la réalité de la gestation. L'exploration manuelle des flancs et du pis ne donnent des résultats sûrs qu'à partir du I20 jour de la gestation. Aussi avons-nous été amenés à explorer par les rayons $\mathrm{X}$ et par laparotomie.

\section{a) Radiographie.}

Les radiographies ont été effectuées avec un appareil ayant les caractéristiques suivantes :

Tension : $72 \mathrm{KV}$.

Courant traversant le tube : Io $\mathrm{mA}$.

Dimensions du foyer du tube à rayons $X: I \times I \mathrm{~mm}$.

Des essais ont été faits, l'animal étant placé dans différentes positions (face dorsale, profil, face ventrale), la distance du foyer à la plaque sensible étant de $70 \mathrm{~cm}$. Mais les meilleurs résultats ont été obtenus à partir de la position dorso-ventrale, la plaque sensible étant appliquée contre la face ventrale de la brebis, maintenue sur une petite table, les pattes pendantes dans le vide. 
Etant donné la faible intensité du courant traversant le tube nous avons été obligés d'opérer avec des temps de pose allant de 5 à 8 secondes. Dans ces conditions, les mouvements respiratoires diminuent la netteté de l'image et il ne nous a pas été possible de déceler la présence du fotus avant le $90^{e}$ jour de la gestation.

Notons que BENZIF (I95I) a pu déceler cette présence à partir du $55^{\mathrm{e}}$ jour. Mais 1'appareil qu'il a utilisé fonctionnait sur $80 \mathrm{KV}$ avec une intensité de $200 \mathrm{~mA}$., ce qui lui a permis de faire des temps de pose très courts de l'ordre de 0,2 sec.

\section{b) Laparotomie exploratrice.}

Pour le diagnostic de la présence des foetus âgés de moins de 90 jours, nous avons procédé par laparotomie après anesthésie générale barbiturique. La laparotomie était suivie d'un traitement à la progestérone pour éviter l'avortement prématuré.

\section{$2 \circ$ Obtention du foetus.}

Vu la rareté du matériel en France, nous avons tenté d'obtenir le fœetus en évitant le sacrifice de la mère. Plusieurs méthodes ont été employées à cette fin.

\section{a) La pose de laminaires dans le col d'utérus.}

Des essais sur 4 Brebis ont permis de constater que la pose des laminaires dans le col utérin est extrêmement difficile, sinon impossible chez la brebis, car le laminaire est assez mou et le col de l'utérus comporte de nombreux replis dirigés vers l'extérieur.

\section{b) L'emploi d'hormones œstrogènes tel que le cycloestrol.}

Comme le montre le tableau I, cette technique ne donne pas de résultats satisfaisants pour le but que nous poursuivons, puisque sur 4 brebis gestantes traitées, deux seulement ont répondu comme prévu. Sur les

$$
\text { TABLEAU I }
$$

Résultats du traitement au cycloestrol.

\begin{tabular}{|c|c|c|c|}
\hline $\begin{array}{l}\text { Numéro } \\
\text { Brebis }\end{array}$ & $\begin{array}{l}\text { Age de } \\
\text { gestation }\end{array}$ & $\begin{array}{l}\text { Dose totale de } \\
\text { Cycloestrol }\end{array}$ & Résultat \\
\hline $\begin{array}{l}1 \\
1 \\
2\end{array} \ldots \ldots \ldots \ldots$ & $\begin{aligned} 136 j \\
71 \\
100 j\end{aligned}$ & $\begin{array}{l}\text { I } 50 \mathrm{mg} \\
\text { I50 } \mathrm{mg} \\
250 \mathrm{mg}\end{array}$ & $\begin{array}{c}\text { Avortement } 24 \mathrm{~h} \text { après la dernière injection. } \\
\text { Avortement sans expulsion ; celle-ci a eu lieu }\end{array}$ \\
\hline $4 \ldots \ldots$ & I35 j & $\begin{array}{l}\mathrm{I} 5 \circ \mathrm{mg} \\
\text { repos }(4 \mathrm{j}) \\
\text { puis too } \mathrm{mg}\end{array}$ & $\begin{array}{c}\text { Avortement jumelé } 8 \text { jours. } \\
\text { injection. }\end{array}$ \\
\hline
\end{tabular}


deux autres, l'une a avorté avec un début de putréfaction du fœetus, et la seconde a avorté d'un fœtus décomposé. De plus, une des brebis traitées et qui avait avorté dans de bonnes conditions, a montré pendant plusieurs mois des symptômes de chaleurs continues. Cependant les trois autres brebis traitées ont mis bas normalement l'année suivante.

Cette méthode s'avère donc trop incertaine pour obtenir des foetus frais au moment souhaité.

\section{c) La césarienne.}

Compte tenu des frais élevés de 1'opération et la nécessité d'effectuer un certain nombre d'opérations pour acquérir une bonne technique, nous avons renoncé à cette méthode.

Finalement, nous avons prélevé les foetus après sacrifice des mères. Les facilités dont nous disposions au C. N. R.Z. par suite de l'existence d'abattoirs nous ont permis d'obtenir des fotus quelques minutes après l'égorgement des mères, ce qui offre un avantage certain pour la qualité des fixations.

\section{$3^{\circ}$ Grefies de la peau.}

Trois sortes de greffes $\left({ }^{1}\right)$ ont été réalisées :

Deux homogreffes réciproques : sur deux agneaux l'un Karakul et l'autre de la race Ile-de-France, tous deux âgés de moins d'une semaine.

Seize autogreffes sur un ensemble de 9 agneaux et adultes Karakul.

Deux syngénésiogreffes: Les greffons ont été prélevés sur deux fœetus, l'un de I I I et l'autre de I32 jours et greffés respectivement sur un agneau et un adulte Karaku1. Nous avons utilisé la technique de la greffe morte.

Dans tous les cas, des greffons de $\mathrm{I} 2 \mathrm{~cm}^{2}$ de surface ont été détachés de l'animal, après anesthésie locale à l'aminocaïne. Dans le cas des autogreffes, les greffons étaient remis en place après avoir subi une rotation de 90 ou $\mathrm{I} 80^{\circ}$, puis maintenus par des coutures marginales.

Les greffons ont été détachés soit entièrement, soit sur les bords en gardant une liaison au centre.

Des prélèvements sur les zones greffées ont été effectués à des intervalles de $\mathrm{I} 5,30,60$ et Ioo jours pour les observations à la loupe binoculaire et les examens histologiques.

\section{C. - TEChNIQUES DE MESURE DES CARACTÉRISTIQUES DES FIBRES}

\section{Mesure du diamètre des fibres.}

Des coupes de poils ont été pratiquées au microtome de Hardy, microtome à main conçu spécialement pour la coupe transversale des

(1) La terminologie que nous utilisons est celle adoptée par R. M. MAY dans "La Greffe "éd. Gallimard, Paris, $195^{2}$. 
poils. Au lieu des coupes minces, nous avons procédé à des coupes de 200 à $300 \mu$. Nous avons obtenu ainsi des portions cylindriques de poils que nous avons étalées sur une lame et montées au baume du Canada. La mesure a été faite par projection (50o fois). C'est la méthode recommandée par la Wool Industries Research Association (WIRA).
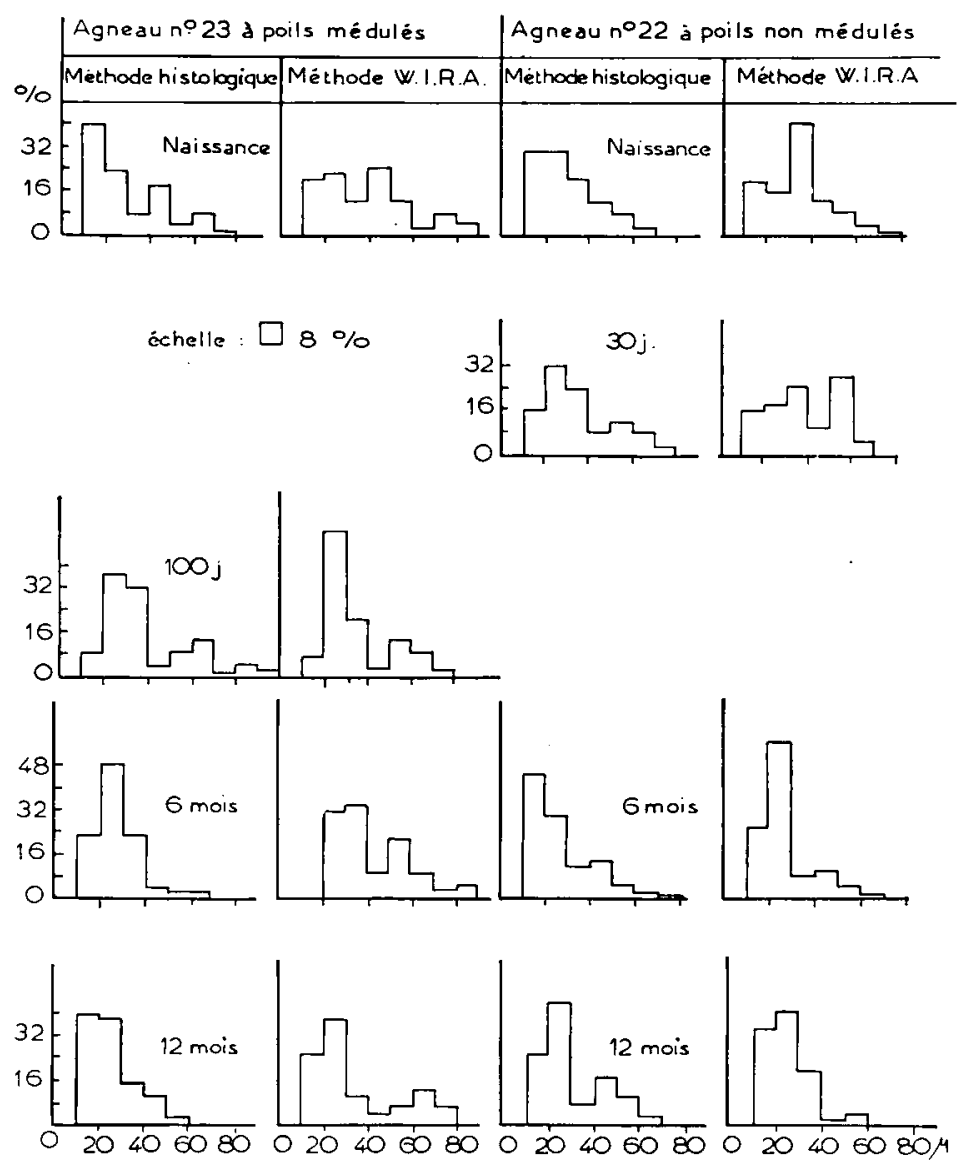

Fig. I. - Histogrammes du diamètre des fibres. Comparaison des résultats obtenus par la méthode sur coupes histologiques et la méthode W. I. R. A.

Nous avons également effectué les mesures sur coupes histologiques minces et parallèles à la surface de la peau, au niveau des glandes sébacées. Chacune de ces méthodes a ses avantages et inconvénients.

\section{a) Méthode W. I. R. A. (Microtome de Hardy).}

Io AvANTAGEs. - Les portions de poils sont dispersées sur la surface de la lame, ce qui permet d'effectuer au hasard les mesures de diamètre (Rougeot, I953).

Les opérations de coupe et de montage sont très rapides. 
$2^{\circ}$ Inconvénients. - Perte des éléments les plus courts qui sont en même temps les plus fins, au cours de l'échantillonnage, ce qui fausse le résultat, mais cet inconvénient est éliminé si toutes les fibres ont plus de $2 \mathrm{~cm}$ de longueur.

Différence de niveau : la coupe ne passe pas au même niveau sur tous les poils. Ainsi pour le même poil, on enregistrerait un diamètre de plus en plus faible au fur et à mesure que la coupe se rapprocherait du sommet. Cet inconvénient est éliminé lorsque toutes les fibres ont plus de $3 \mathrm{~cm}$ de longueur permettant des coupes à un niveau assez éloigné du sommet.

\section{b) Mesure sur coupes histologiques.}

Io Avantages. - Pas de perte au moment de l'échantillonnage. Les mesures se font au même niveau des poils, celui des glandes sébacées ce qui est important étant donné le caractère conique du poil près du sommet.

$2^{\circ}$ Inconvé,nien'ts. - Dans les cas de polymorphisme exagéré de la section droite des poils de certains individus (section triangulaire, ovale, polygonale) on hésite devant le choix de l'axe ou du diamètre à mesurer et les mesures ne sont plus effectuées au hasard.

Difficulté d'obtenir des coupes transversales des poils avec les peaux tannées qui se coupent mal et portent des poils très inclinés.

Nécessité des préparations histologiques qui sont des opérations relativement longues.

La figure I montre la comparaison des deux méthodes pour les mêmes animaux. On remarque nettement que par la méthode W.I. R. A. (méthode ordinaire) il se produit une perte au moment de l'échantillonnage chez l'agneau, ce qui se traduit par un déplacement du mode vers les diamètres les plus forts. Au contraire, après le premier mois, les courbes obtenues par les deux méthodes sont comparables. Ainsi la méthode de la W.I. R. A. ne convient pas pour le fœtus et le jeune agneau, ce qui nous a conduit à employer, dans ce cas, la méthode histologique.

\section{2o Mesure de la Iongueur des fibres.}

Trois procédés ont été essayés :

\section{a) Méthode des pinces.}

La fibre est tendue entre les mors de deux petites pinces et mesurée avec une règle graduée. Cette méthode valable pour des fibres longues d'une toison adulte ne saurait convenir pour les poils et duvet de 1'astrakan mesurant de I à I7 millimètres. On s'imagine aisément les difficultés à tenir entre deux pinces des petites fibres incurvées, à les dérouler et à les tendre également sans risque de les casser. Les résultats obtenus avec 
une telle méthode ne sauraient donc être retenus. Pourtant, elle avait été utilisée par TÄNZER (I928) puis par NICOV (I930).

\section{b) Méthode de Halle.}

A 1'Institut Zootechnique de Halle (Allemagne) une méthode très rapide a été proposée pour mesurer la longueur des poils d'une boucle sans raser celle-ci. Il suffit d'introduire sous la boucle l'extrémité d'une spatule en bois recouverte de papier millimétré et d'appliquer la boucle sur cette surface millimétrée.

En réalité, il s'agit donc d'une simple estimation approximative de la longueur moyenne des poils et non de la mesure des longueurs individuelles. De plus, avec cette méthode, on ne peut pas se faire une idée de la longueur du duvet car seuls les poils sont pris en considération. Cette méthode couramment pratiquée en Afrique du Sud-Ouest était trop imprécise pour être utilisée.

\section{c) Méthode par projection.}

Nous avons alors mis au point une méthode sûre, précise et très pratique qui est la suivante :

La peau est rasée sur quelques $\mathrm{mm}^{2}$ par un seul passage d'une lame de rasoir. La totalité des fibres rasées est prélevée et montée au baume du Canada très liquide sur plusieurs lames porte-objets, puis recouverte d'une lamelle. Les fibres sont soigneusement réparties et séparées autant que possible les unes des autres pour éviter des enchevêtrements compliqués. L'image est projetée sur un verre dépoli ou mieux encore, sur un verre ordinaire contre lequel on applique une feuille de papier calque. Nous avons réalisé cette installation par un montage spécial et en nous servant d'un lecteur de microfilm comme projecteur. Une combinaison du montage permet d'avoir à la portée de la main la lame et l'écran de projection afin d'éviter les déplacements fastidieux qui pourraient en même temps être la source d'erreurs. L'image agrandie ro fois est mesurée avec un curvimètre donnant directement les résultats en millimètres. Toutes les mesures de longueur ont été effectuées avec cette méthode.

\section{$3^{\circ}$ Observation des écailles des fibres.}

Étant donné l'opacité des poils Karakul par transparence et la difficulté de leur observation par éclairage vertical ou latéral, nous avons procédé à des empreintes sur gélatine (solution à Io p. IOO).

\section{$4^{0}$ Forme de la section droite des fibres.}

Les observations ont été faites sur des coupes histologiques parallèles à la surface de la peau au niveau des glandes sébacées ou bien sur des coupes minces des fibres obtenues après congélation. 


\section{5o Médullation des fibres.}

I a détermination du pourcentage des fibres médullées a été effectuée sur les coupes ayant servi à l'examen de la forme de la sectión des fibres.

\section{$6^{\circ}$ Détermination du rapport $S / P$.}

I1 s'agit du rapport du nombre de follicules ou de fibres secondaires sur celui des follicules ou des fibres primaires.

I,e rapport $\mathrm{S} / \mathrm{P}$ a été déterminé sur des coupes histologiques parallèles à la surface de la peau, qui seules permettent de distinguer, de façon précise, les follicules primaires des follicules secondaires. Mais chaque fois que la méthode histologique s'est avérée impossible, notamment en ce qui concerne les peaux tannées, nous avons déterminé ce rapport d'après l'histogramme de la longueur des fibres. En effet, la différence de la longueur entre les fibres primaires et les fibres secondaires est telle que celles-ci constituent sur 1'histogramme deux populations nettement distinctes.

Pour la clarté du texte, quand le rapport $S / P$ est établi en dénombrant les fibres nous indiquerons $S / P$ fibres. Lorsqu'il s'agira du rapport du nombre des follicules secondaires au nombre des follicules primaires, on devra tenir compte du fait que tous les follicules ne sont pas au même état fonctionnel : il peut y avoir des follicules ne possédant pas encore de fibres, de telle sorte qu'on est amené à distinguer deux rapports $S / P$ folliculaires selon que l'on dénombre tous les follicules ou seulement les follicules avec fibre différenciée. Nous appellerons le premier, S/P folliculaire I (S/P I), et le second, $\mathrm{S} / \mathrm{P}$ folliculaire $2(\mathrm{~S} / \mathrm{P} 2)$.

\section{$7^{\text {o }}$ Détermination de la densité pileuse.}

Cette détermination a été faite sur des coupes histologiques parallèles à la surface de la peau passant au niveau des glandes sébacées. Les résultats se rapportent alors au $\mathrm{mm}^{2}$ de la coupe sur lame. Pour les peaux tannées, le comptage des fibres a été fait sous la loupe binoculaire après avoir rasé la peau, un micromètre à carreaux ayant été préalablement placé dans l'oculaire de la loupe.

\section{go Mesure de l'épaisseur de la peau.}

L'épaisseur de 1'épiderme a été mesurée sur des coupes histologiques perpendiculaires à la surface de la peau, à l'aide du microscope muni d'un micromètre oculaire. Il en est de même pour l'épaisseur du derme des fœtus. Pour les peaux d'agneaux fraîches, brutes et tannées, l'épaisseur de celles-ci a été mesurée au Palmer (au $\mathrm{I} / \mathrm{IOO}^{\mathrm{e}}$ de $\mathrm{mm}$ ) après avoir soigneusement rasé les fibres. 


\section{9o Mesure de la surface des peaux.}

Cette mesure a été effectuée à l'aide du papier millimétré.

\section{$10^{o}$ Appréciation du lustre.}

Pour apprécier le lustre des peaux d'agneaux, nous avons essayé de standardiser les mesures. Pour ce faire, nous avons éclairé les peaux toujours à $45^{\circ}$, la lampe (S) et la peau formant un système solidaire dont l'ensemble tourne autour d'un axe vertical. L'observation a été faite successivement à $45^{\circ}$ et à $90^{\circ}$ (fig. 2). Le rapport de $1^{\prime}$ observation à $45^{\circ}$
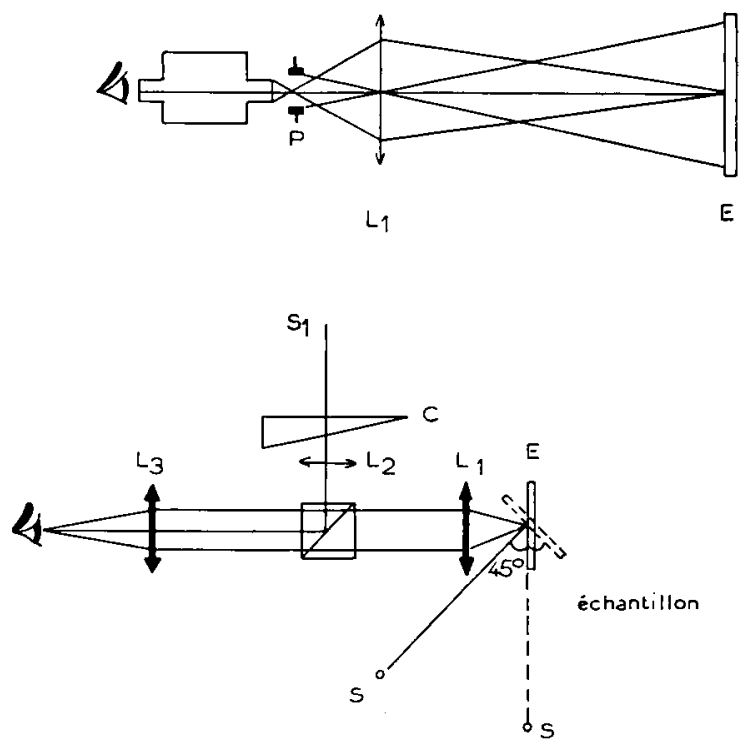

Fig. 2. - Dispositif optique du nitomètre.

à l'observation normale $\left(90^{\circ}\right)$ caractérise le brillant (lustre). Ces observations ont été faites dans les conditions normales de la vision de l'œil et pour cela les deux conditions suivantes ont été remplies :

- éclairer une plage assez grande $(5 \mathrm{~cm}$ de diamètre) ;

- utiliser une source lumineuse dont le spectre correspond au rayonnement solaire moyen.

Nous avons choisi une lampe à filament de tungstène dont la température de couleur est de $2854^{\circ} \mathrm{K}$ (absolus). Un filtre approprié modifie la lumière incidente en lui donnant une composition spectrale sensiblement égale à celle de la lumière du jour solaire moyen. Le filtre utilisé est soit le filtre liquide de Davis et Gibson, soit un filtre Corning de $4,85 \mathrm{~mm}$ d'épaisseur.

I e filtre de Davis et Gibson est constitué par une cuve composée 
de trois lames planes polies de borosilicate-crown de 2,5 $\mathrm{mm}$ d'épaisseur et séparées par deux plaques de verre noir finement doucies de Io $\mathrm{mm}$ d'épaisseur de sorte que chaque solution aura I cm d'épaisseur. Les deux compartiments de la cuve sont remplis respectivement de solutions $\alpha$ et $\beta$ dont la composition est la suivante :

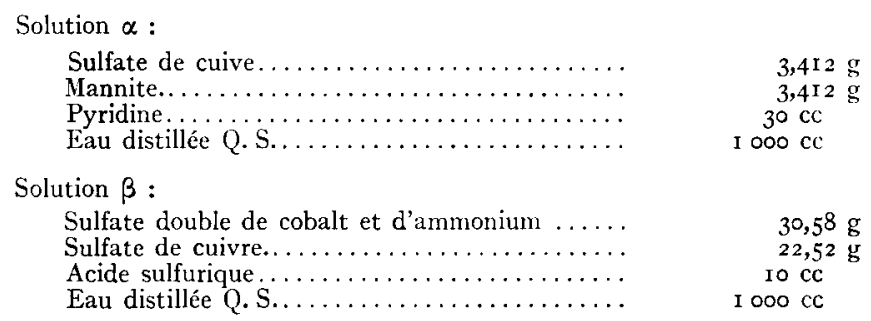

Les dispositifs préconisés sont d'une part le nitomètre et d'autre part la méthode photoélectrique.

\section{a) Nitomètre $=$ Luminance-mètre.}

Le nitomètre utilisé est un appareil " Jobin et Yvon " saṇs écran diffusant. L'œil de l'observateur placé à la pupille de sortie du nitomètre observe deux plages inégales de luminance différente : l'une provenant du nitomètre lui-même, l'autre de l'objet éclairé.

La lumière émise de la source $S_{1}$ traverse un coin photométrique dont le déplacement modifie son intensité, et tombe sur la partie centrale du cube de Lummer où elle subit la réflexion totale et forme une petite plage lumineuse dont l'intensité est réglable. Le reste du cube laisse traverser la lumière provenant de l'échantillon (fig. 2).

L'échantillon $\mathrm{E}$ est éclairé sur une plage de $5 \mathrm{~cm}$ de diamètre avec une incidence de $45^{\circ}$. I a lentille $L_{1}(f=3 \mathrm{~cm})$ donne l'image de cette plage en un plan situé sur la pupille d'entrée $P$ du système optique. La lumière provenant de l'échantillon va traverser le cube de Lummer et former une plage lumineuse.

On égalise la luminance des deux plages (celle provenant de $\mathrm{S}_{1}$ et celle de l'image de l'échantillon éclairée par S) par un déplacement du coin, et on lit le chiffre correspondant sur le tambour gradué.

\section{b) Méthode photoélectrique.}

Le dispositif d'éclairement de l'échantillon est le même que pour la méthode précédente, mais on remplace le nitomètre par une cellule photoélectrique.

On utilise une cellule à couche d'arrêt dont la sensibilité spectrale est sensiblement la même que celle de l'œil. La cellule est associée à un galvanomètre de grande sensibilité $\left(\mathrm{IO}^{-9} \mathrm{~A} / \mathrm{mm}\right)$. 


\section{DÉVELOPPEMENT ET CARACTERISTIQUES DU SYSTEME PILEUX}

\section{HISTORIQUE}

Le développement de la toison des Ovins a été l'objet de nombreux travaux, mais ceux-ci ont porté surtout sur d'autres races que le Karakul.

Ainsi, Gal,PIN (I935) observe chez la race Romney que la formation de follicules commence vers le $49^{\mathrm{e}}$ jour sur la tête, pour s'étendre à partir du $64^{\mathrm{e}}$ jour sur toute la surface du corps. Chez la race Kirghiz (Steatopygae) selon TerenjeVA (I937) la formation des follicules commence au $45-50^{\mathrm{e}}$ jour sur la tête pour s'étendre au $80-85^{\mathrm{e}}$ jour sur le corps entier. CARTER (I943) dans son travail sur le développement des populations folliculaires chez le Mérinos constate que les follicules primaires apparaissent sur le flanc vers le $65^{\mathrm{e}}$ jour et les secondaires vers le $86^{\mathrm{e}}$ jour de la vie fœetale. Dromidova (I954) publie les résultats de ses recherches sur le développement embryonnaire de la peau et de la laine chez les Mérinos croisés soviétiques. Elle note en particulier une relation entre la variation de l'épaisseur de l'épiderme et la formation de la fibre: elle observe une augmentation suivie d'une diminution importante de l'épaisseur de l'épiderme au moment de l'éruption des fibres à la surface de la peau qui a lieu au $\mathrm{IOo}^{\mathrm{e}}$ jour au niveau de l'omoplate.

Les travaux de MEIJERE en I894 montrant que les follicules pileux des Mammifères sont arrangés en groupes définis, construits à partir de 3 follicules principaux, furent poursuivis et développés par HOFER (I9I4) chez le chat, GibBs (I938) et HaRdy (I946) chez les Marsupiaux. Chez les Ovins le développement et la constitution des groupes folliculaires furent étudiés à partir de fœetus de différents âges par WILDMaN (I932) chez plusieurs races ovines anglaises, par GaLPIN (I935) chez le Romney et par DUERDEN (I939) chez le Mérinos.

Wildman et CarTer (I939) proposent une terminologie des follicules pileux chez les Mammifères et divisent les follicules en deux groupes: les primaires et les secondaires. Les follicules primaires se forment par " trio " et sont appelés $\mathrm{xXx}$ ou $\mathrm{yYy}$ suivant qu'ils sont plus ou moins précoces dans leur apparition. Les follicules secondaires apparaissent plus tard et sont caractérisés par l'absence de glande sudoripare.

CARTER (I943) entreprend l'étude biométrique des populations folliculaires à différents âges chez le fotus Mérinos. Cette étude l'amène à introduire la notion du rapport $\mathrm{S} / \mathrm{P}$, rapport du nombre des follicules 
secondaires au nombre des follicules primaires, qui caractérise les populations folliculaires.

Les termes primaire, secondaire, tertiaire, etc... ont également été employés par de nombreux auteurs. Mais la plupart des publications postérieures à I939 ont adopté la terminologie de WILDMAN et CARTER. Quant aux publications antérieures à cette date, notons que WILDMAN lui-même (I932), GALPIN (I935) et DUERDEN (I939) ont appelé follicules primaires et secondaires, $X$ et $Y$, des follicules qui sont tous considérés aujourd'hui comme primaires. La terminologie allemande, qu'il s'agisse de Mittelhaar, Stammhaar (ToLdT, Igri et HöFER (Igr4), ou de Leithaar (SPöTTEL et TäNzER, I923), sont tous également des fibres primaires; tandis que Beihaar et Gruppenhaar sont considérés comme des fibres secondaires.

I.es follicules secondaires eux-mêmes ont été divisés en deux catégories dans la nouvelle nomenclature de HARDy et L,YNE (I956) cenx qui proviennent directement d'une invagination épidermique (follicules secondaires originels) et ceux qui dérivent d'un autre follicule secondaire (follicules secondaires dérivés).

En ce qui concerne la race Karakul, les études sur le développement du follicule et la constitution des groupes folliculaires sont relativement peu nombreuses. Selon Nikoljskr (I932) les premiers indices de la formation des follicules pileux chez le Mouton Karakul s'observent déjà sur la tête de l'embryon de 60 jours. L'auteur indique que la totalité de la surface de la peau du foetus Karakul ne se couvre de follicules qu'au $75^{\mathrm{e}}$ jour.

MARGOIENA (I954) observe une augmentation continue de l'épaisseur de la peau, avec l'âge, chez le fotus et l'agneau Karakul. Il trouve chez l'agneau que le rapport $\mathrm{S} / \mathrm{P}$ est compris entre 2 et 5 et cons. tate l'absence de formation des ébauches folliculaires après la naissance.

Ainsi, bien que les processus de la genèse du système pileux soient les mêmes chez toutes les races ovines, il existe des différences en ce qui concerne les dates d'apparition et les caractéristiques des fibres. En outre, la formation et l'évolution des follicules, assez bien connues chez le Mérinos et certaines races ovines britanniques, ne sont que peu étudiées chez le Karakul. Nous avons donc entrepris l'étude de la peau et du poil, chez cette race, depuis le $45^{\mathrm{e}}$ jour de la vie fotale jusqu'à 1'âge adulte. Dans les pages qui suivent nous exposerons les résultats de nos recherches sur les modifications de la peau au cours de la vie foetale, la formation et l'évolution des follicules et les caractéristiques des fibres chez le fœus et 1'agneau à différents âges. 


\section{RÉSULTATS DE RECHERCHES}

\section{A. - MODIFICATIONS DE LA PEAU AU COURS DE LA VIE FEETALE}

$\mathrm{I}_{1}$ a peau du mouton Karakul a un épiderme mince constitué uniquement par l'épithélium de Malpighi recouvert d'une couche desquamante de stratum corneum. Au contraire, au début du développement embryonnaire on distingue plusieurs couches de cellules à noyaux sphériques ou aplatis entre l'épithélium malpighien et le stratum corneum.

$\mathrm{Au} 45^{\mathrm{e}}$ jour de la vie foetale, alors qu'il n'existe encore aucune trace de follicule et de pigment visible, la peau possède un épiderme bien différencié, formé de trois assises de cellules. I'épiderme et le derme ont les mêmes épaisseurs au niveau de la tête et au niveau du flanc; ils mesurent environ 8 et II $5 \mu$ respectivement et contiennent des noyaux cellulaires sphériques ou plus ou moins aplatis de 6 à $7 \mu$ de diamètre.

Au $56^{\text {e }}$ jour, la peau mesure 200 : d'épaisseur sur le flanc. Au niveau de la tête on assiste, dans le derme, à une invagination des follicules accompagnés de grains de mélanine, tandis qu'au niveau du flanc ni les. follicules, ni la mélanisation ne sont pas encore apparus. Les noyaux de l'épiderme sont sphériques ou aplatis et mesurent de 5 à Io $u$ de diamètre. Ceux du derme sont ovoïdes.

Au niveau du flanc, au 68 e jour, l'épiderme est constitué de 4 couches. cellulaires et mesure Io $\mu$ d'épaisseur. I'épaisseur du derme est de $270 \mu$. et montre l'invagination des follicules pileux. Ceux-ci sont plus développés sur la tête où ils atteignent une longueur de 80 à Ioo $\mu$.

Au $75^{\mathrm{e}}$ jour, l'épiderme a atteint une épaisseur de $12,5: \leftrightarrow$ aut niveau du flanc. I a couche de Malpighi est composée de deux assises cellulaires à noyaux sphériques. Au-dessus de cette couche, on compte trois assises de cellules à noyaux aplatis dans un plan parallèle à la surface de la peau. Le collagène du derme est constitué par des fibres assez laches prenant mal la coloration. Les follicules sont situés dans le tiers supérieur de l'épaisseur du derme.

L'épiderme et le derme continuent à s'épaissir de sorte qu'au $85^{\mathrm{e}}$ jour ils mesurent respectivement $I 7,5$ et $402 \mu$ au niveat du flanc et $I 7,5$. et $642 \mu$ au niveau de la tête. L'épiderme est constitué de 5 assises cellulaires. Sur la tête, les deux assises les plus externes commencent à se: détacher. A ce niveau, on assiste dans le derme, à certaines modifications : les noyaux sphériques en augmentation de taille ont acquis des formes diverses (ovoïdes, étirés...). La présence de certains noyaux de forme très allongée, de I2 à $15 \mu$ de longueur, montre la différenciation du muscle arrecteur. 
Au $95^{\mathrm{e}}$ jour, l'épaississement de l'épiderme s'est poursuivi: l'épiderme et le derme mesurent respectivement 30 et $690 \mu$ au niveau du flanc. Il n'existe pas encore de muscle arrecteur à cet endroit du corps où il apparait peu avant le $I I^{e}$ jour.

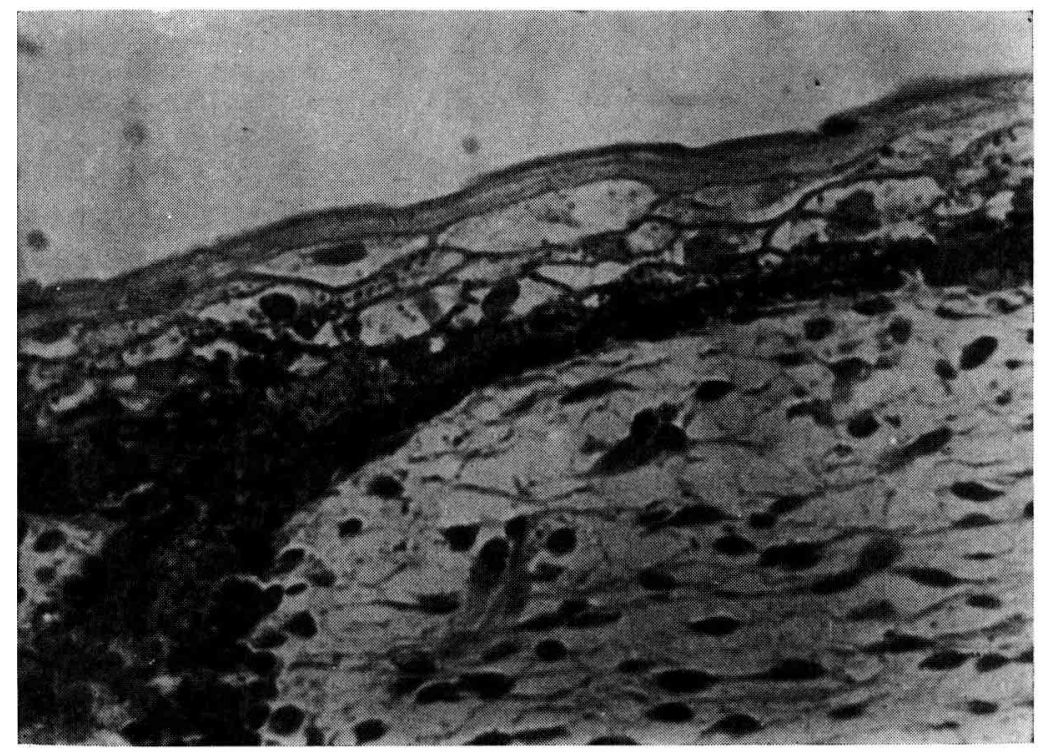

FIG. 3. - Epiderme de l'embryon Karakul de I Io jours (flanc) ayant atteint son maximum d'épaisseur (35 i).

A cet âge, sur le flanc, la peau mesure goo $\mu$ d'épaisseur. L'épiderme, constitué de 6-7 assises de cellules bien visibles, atteint une épaisseur de $35 \mu$ (fig. 3). Les noyaux des cellules épidermiques s'aplatissent à mesure que l'on s'approche de la surface de la peau. Ceux du derme ont une forme ovoïde plus ou moins allongée. Les follicules pileux atteignent environ $300 \mu$ de longueur et présentent des fibres dont l'état de kératinisation est plus ou moins avancé selon qu'on se rapproche ou s'éloigne des lignes dorsale et latérale (fig. 8).

L'embryon de $\mathbf{I} 2 \mathrm{I}$ jours possède au niveau du flanc un épiderme constitué par l'épithélium de Malpighi (formé de 2 assises cellulaires) et par un stratum corneum. L'épiderme à cet âge ne mesure plus que ${ }^{3} \mu$. $L$ 'épaisseur totale de la peau est de I $450 \mu$ dont les $3 / 4$ sont envahis par les follicules.

Chez l'agneau à la naissance, l'épaisseur au niveau du flanc, est de I4-I6 $\mu$ pour l'épiderme, et de I 200-I $920 \mu$ pour le derme. Chez l'agneau, l'épaisseur de la peau a été également mesurée au Palmer; les valeurs obtenues à différents niveaux du corps sont indiquées sur le tableau II (I2 agneaux contrôlés). 
TABLEAU II

Epaisseur moyenne de la peau chez l'agneau Karakul mesurée au Palmer $\grave{a}$ différentes parties du corps.

\begin{tabular}{|c|c|}
\hline Région du Corps & Epaisseur en $\mathrm{mm}$ \\
\hline Tête.... & $I, 5^{\circ}$ \\
\hline Cou .......... & $\mathrm{I}, 25$ \\
\hline Dos .................. & 1,62 \\
\hline Croupe $\ldots \ldots \ldots \ldots \ldots$ & $\mathrm{I}, 42$ \\
\hline Ventre................ & 0,87 \\
\hline Queue $\ldots \ldots \ldots \ldots \ldots$. & 1,43 \\
\hline
\end{tabular}

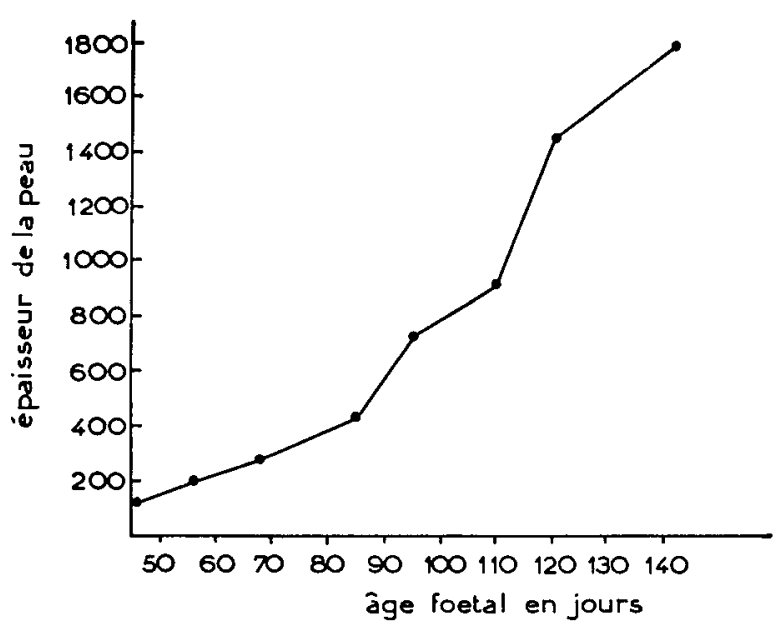

Fig. 4. - Variation avec l'âge de l'épaisseur de la peau de l'embryon Karakul.

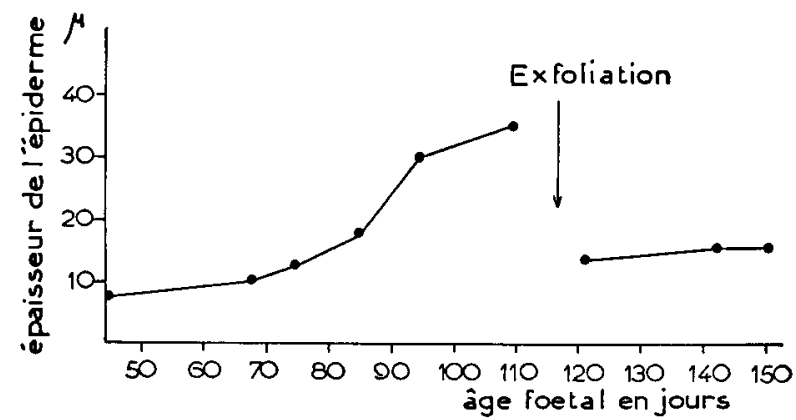

FIg. 5 - Variation avec l'âge de l'êpaisseur de l'épiderme de l'embryon Karakul au niveau du flanc.

Le tableau ci-dessus montre que l'épaisseur moyenne de la peau est beaucoup plus faible dans la région ventrale $(0,87 \mathrm{~mm})$ que sur les régions dorsales (cou, dos, croupe, queue). Toutefois, l'épaisseur de la peau peut varier du simple au double d'un agneau à l'autre.

La peau du fotus, de l'agneau et de l'adulte Karakul, est très riche 
en fibres élastiques au niveau du stratum intermedium (mises en évidence par la Fuchsine paraldéhyde) qui s'étend depuis les glandes sébacées jusqu'au niveau d'insertion du muscle arrecteur. Ite reste est riche en collagène qui est abondant dans le stratum sub-épithélial et le stratum reticulum. Il n'y a pas de tissus adipeux dans la peau de l'agneau Karakul.

Nous venons de voir que la peau s'épaissit d'une façon continue au cours de la vie embryonnaire (fig. 4). Toutefois, l'épiderme et le derme n'évoluent pas de la même manière, le premier subissant à partir du IIo jour une exfoliation qui ramène son épaisseur de 35 à $13 \mu$ (fig. 5). L'épiderme qui comptait 6-7 assises de cellules au $\mathrm{Iro}^{\mathrm{e}}$ jour n'en compte plus que 3 au $\mathrm{I} 2 \mathrm{I}^{\mathrm{e}}$ jour. A partir de cet âge, l'épaisseur de l'épiderme reste relativement constante jusqu'à l'âge adulte. Le tableau III montre la variation de l'épaisseur totale de la peau et de celle de l'épiderme chez les fotus de différents âges.

\section{TABLEAU III}

Variation avec l'âge de l'épaisseur totale de la peau et de l'épaisseur de l'épiderme chez le toetus Karakul.

\begin{tabular}{|c|c|c|c|c|c|}
\hline \multirow{3}{*}{\multicolumn{2}{|c|}{ Age fœtal en jours }} & \multicolumn{4}{|c|}{ Epaisseur en : } \\
\hline & & \multicolumn{2}{|c|}{ Epiderme } & \multicolumn{2}{|c|}{ Epiderme + Derme } \\
\hline & & Tête & Flanc & Tête & Flanc \\
\hline $\begin{array}{l}45 \\
45\end{array}$ & $\ldots \ldots$ & 8 & 7,5 & I 20 & I 25 \\
\hline $\begin{array}{l}50 \\
68\end{array}$ & ……? & $\overline{I 2,5}$ & 10 & $\begin{array}{l}200 \\
240\end{array}$ & $\begin{array}{l}200 \\
280\end{array}$ \\
\hline 75 & & I2,5 & 12,5 & 420 & - \\
\hline 85 & $\ldots \ldots \ldots \ldots$ & 17,5 & $\mathrm{I} 7,5$ & 660 & 420 \\
\hline 95 & $\ldots \ldots \ldots \ldots$ & - & 30 & - & 720 \\
\hline IIO & $\ldots \ldots \ldots \ldots$ & - & 35 & - & 900 \\
\hline I 2 I & $\cdots \cdots$ & - & I3 & - & I $45^{\circ}$ \\
\hline $\mathbf{I}_{50}$ & ${ }_{1}, \ldots$, & - & $14-16$ & 一 & $1200-1920$ \\
\hline
\end{tabular}

La chute brusque de l'épaisseur de l'épiderme est due à l'exfoliation du substratum corneum et de plusieurs assises cellulaires voisines. Elle est moins importante sur la tête que sur le flanc. Cette chute de l'épaisseur de l'épiderme coïncide avec l'éruption des fibres à la surface de la peau. Il y a donc, chez le fœetus Karakul, une relation étroite entre l'éruption des fibres et la chute de l'épaisseur de l'épiderme comme l'avait constaté Dromidova (1954) chez le Mérinos.

\section{B. - FORMATION ET ÉvOLUTION DU FOLLICULE PILEUX}

\section{$1^{\circ}$ Anatomie du follicule pileux.}

On appelle follicule pileux la portion du poil ou de la laine implantée dans la peau, avec ses différentes gaines et annexes (fig. 6). 


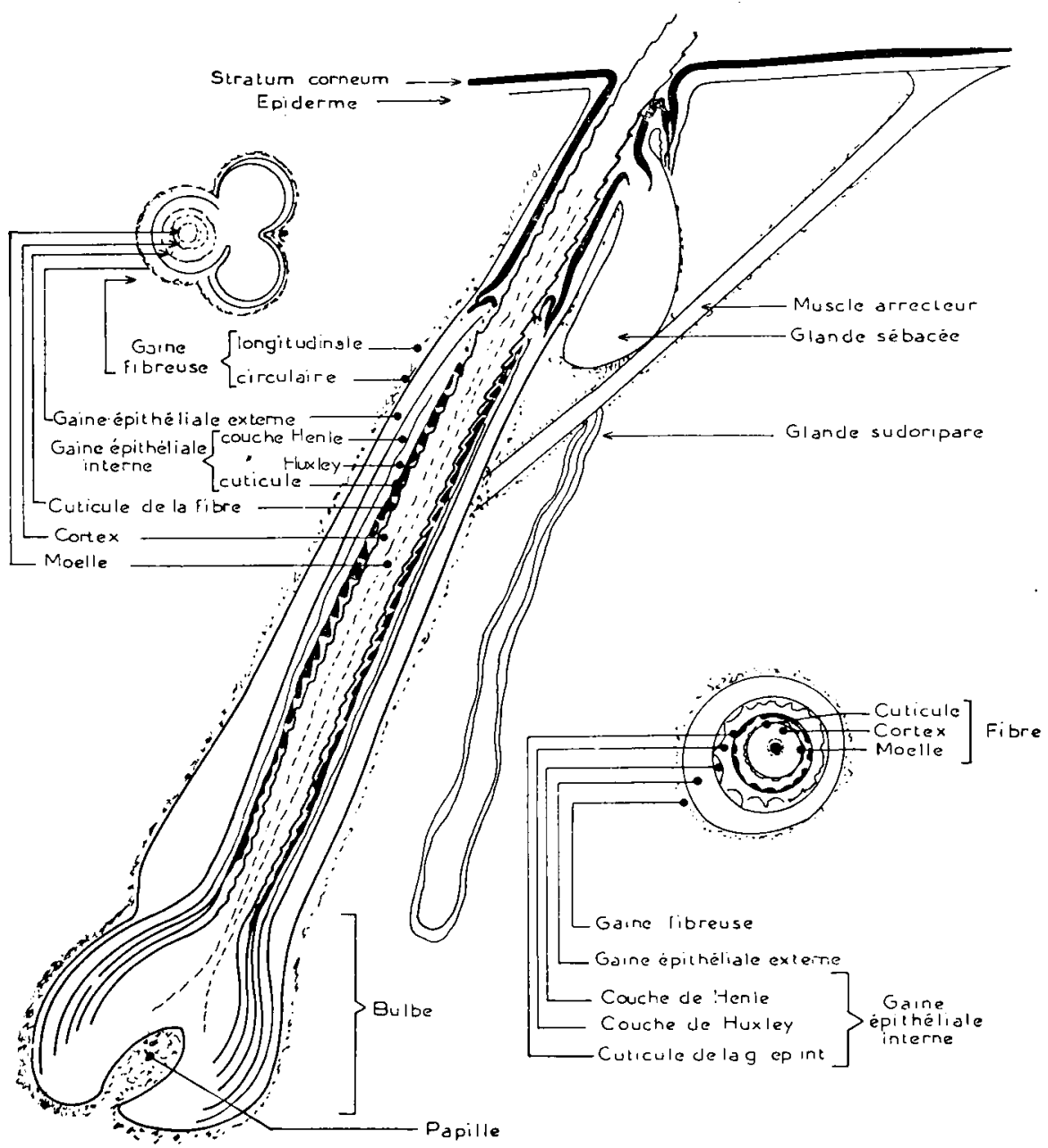

Fig. 6. - Schéma du follicule pileux dans la race Karakul. Au centre : Coupe longitudinale du follicule. - A gauche : Coupe transversale au niveau des glandes sébacées. - A droite : Coupe transversale au-dessus du bulbe.

On distingue, de la périphérie au centre :

- gaine fibreuse,

- gaine épithéliale externe,

- gaine épithéliale interne,

- cuticule,

- cortex,

- mœlle.

Le follicule pileux chez le Karakul est, dans ses grandes lignes, semblable à celui des autres races ovines, quoique présentant quelquefois, dans les détails, certaines particularités. 


\section{a) Le bulbe.}

Chez le mouton Karakul, le bulbe est très souvent déjeté de sorte que son axe forme un angle plus ou moins obtus avec celui du follicule. Cette déflexion est en général située dans le plan de l'inclinaison du follicule. Contrairement à ce qu'avait indiqué PFEIFER (I928), nous tenons à noter que cette déflexion n'est pas spéciale au Karaktul puisque nous l'avons observée aussi chez les races Ile-de-France, Bizet et Limousine. Wirdman (I932) et AUBer (I95I) l'ont également constatée chez les races ovines britanniques.

La base du bulbe est le siège de multiplications cellulaires intenses; elle est formée de cellules indifférenciées, avec une accumulation très dense de mélanine. A un niveau plus élevé, des rangées cellulaires plus ou moins distinctes se dessinent et on observe déjà l'ébauche des différentes couches de follicule, chacune étant représentée par une seule assise cellulaire.

\section{b) Gaines épithéliales.}

Au début de sa formation le follicule étant droit et présentant un axe de révolution, l'épaisseur de la gaine épithéliale externe est régulière tout autour du follicule. Cette régularité peut s'observer également chez les follicules droits adultes (mûrs). Cependant, dans la majorité des cas le bulbe étant déjeté, on observe un épaississement de la gaine épithéliale externe du côté concave de la déflexion du bulbe au-dessus de celui-ci. Cet épaississement sera d'autant plus important que la déflexion du bulbe sera plus prononcée. L'asymétrie ainsi provoquée est limitée à la partie proximale et médiane du follicule. Il en est de même chez les autres races ovines que nous avons observées.

La gaine épithéliale interne présente également une asymétrie, dans le même sens que la gaine épithéliale externe de sorte que ses parois sont plus épaisses du côté de la déflexion du bulbe.

La fibre comprend en général 3 parties qui sont de l'extérieur à l'intérieur : 1a cuticule, le cortex et la mœlle (fig. 6). La cuticule est formée d'écailles imbriquées à la façon des tuiles d'un toit et recouvre toute la surface de la fibre protégeant le cortex. Le cortex est formé par une masse compacte de cellules en forme de fuseau et constituées entièrement de kératine. Au centre, la moelle trouve son origine à partir de la région centrale du sommet de la papille. Les cellules élaborées par cette zone se vacuolisent de bonne heure et les noyaux disparaissent progressivement sans aucune élongation. Les trames de cytoplasme desséché délimitent des cavités qui se remplissent de gaz. La moelle n'existe pas au sommet des fibres médullées par conséquent sa différenciation débute avec retard sur la différenciation du cortex. C'est pourquoi chez l'agneau 
Karakul les poils, qui sont encore de faible longueur, ne sont pas toujours médullés. Ainsi nous avons observé un pourcentage de poils médullés très variable suivant les individus. La moelle peut être continue ou discontinue et de dimensions très variables. Notons que les fibres fines appelées laine ne sont pas médullées.

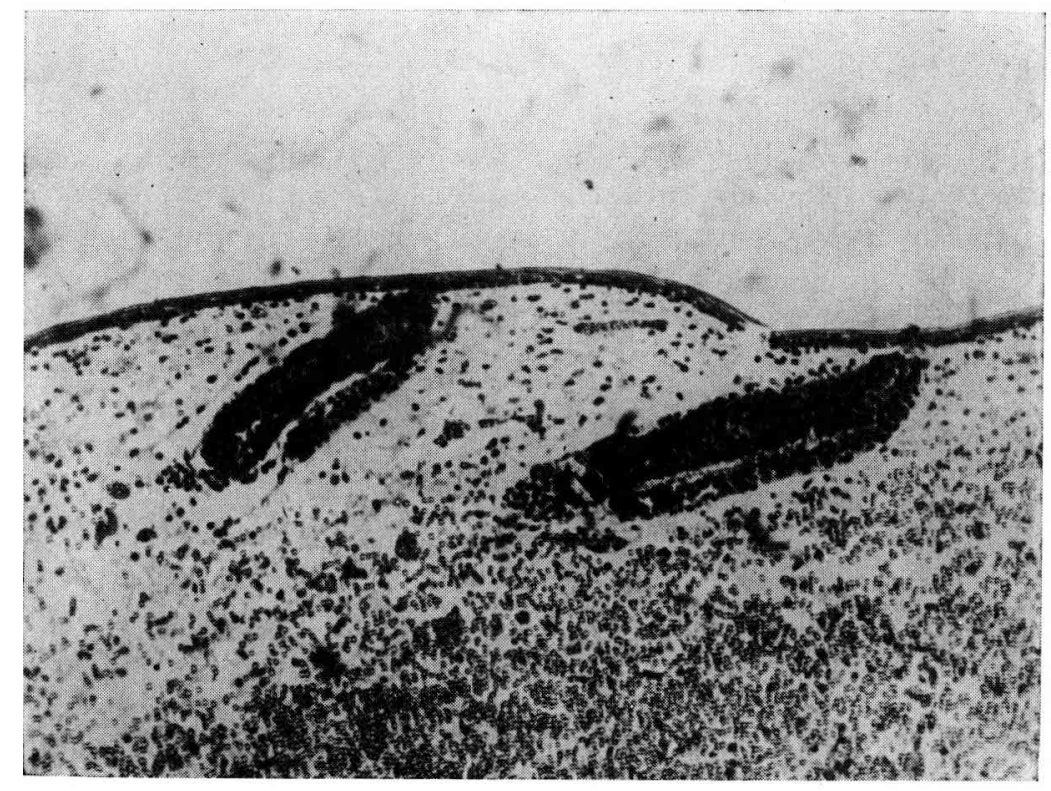

Fig. 7. - Fotus Karakul de 76 jours montrant l'inclinaison du follicule et l'emplacement de la glande sudoripare.

2o Développement du follicule pileux.

\section{a) Apparition.}

Les follicules se développent suivant le même schéma sur tout le corps du fœtus mais prennent naissance à différentes époques suivant leur localisation.

Le follicule débute par une invagination de l'épiderme dans le derme. Le bourgeon ainsi formé et précédé d'un amas de cellules dermiques pénètre progressivement dans les profondeurs du derme.

Au début de sa formation, ce bourgeon ne forme qu'une masse amorphe sans aucune différenciation de couches et est implanté obliquement par rapport à la surface de la peau (fig. 7).

Cette obliquité ne se fait pas au hasard mais elle a tendance à se diriger, dans l'ensemble, vers la queue et vers les flancs.

Chez l'embryon Karakul nous avons pu observer aisément cette 
invagination, sans aucune coloration, grâce à l'accumulation de mélanine contenue dans les éléments épidermiques.

\section{b) Différenciation.}

Alors que l'ébauche du follicule continue à s'enfoncer dans le derme, la glande sudoripare apparaît sous la forme d'un bourgeon situé sur le côté inférieur du follicule, tout près de l'épiderme.

Plus tard, les glandes sébacées se différencient à partir d'autres bourgeons qui sont apparus en dessous du point de départ de la glande sudoripare. Le follicule continue à s'allonger, son extrémité s'élargit, s'invagine de façon à entourer l'amas d'éléments dermiques qui le précède. Ainsi se forment le bulbe et la papille dermique (fig. 7 bis).

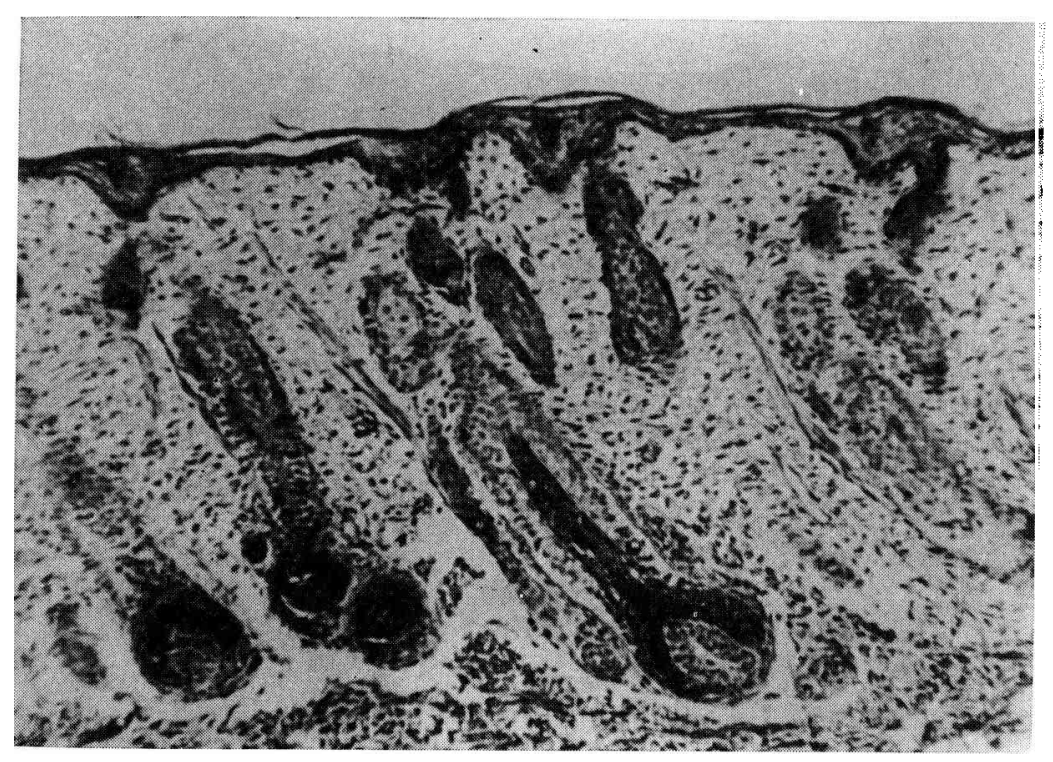

FIG. 7 bis. - Foctus Karakul de 85 jours (tête) montrant l'invagination du bulbe, la formation de la papille et le début de la différenciation du poil.

La partie inférieure $d u$ bulbe est le siège d'une multiplication cellulaire intense et de la différenciation de plusieurs assises cellulaires qui donneront naissance aux différentes couches du follicule. Aussi l'a-t-on appelée matrice pileuse ou zone génératrice.

Les mélanocytes se multiplient également de sorte qu'une accumulation de mélanine se produit dans le bulbe et rend l'observation microscopique très difficile.

Le bulbe, symétrique au début, devient asymétrique, ce qui provoque une légère déflexion du follicule au niveau immédiatement au-dessus 
du bulbe. Cependant cette déflexion ne prendra toute son ampleur que bien plus tard.

L'activité du follicule aboutit à la formation d'une fibre à partir des cellules centrales de la matrice pileuse.

La gaine épithéliale interne se différencie conjointement avec la fibre. Fille prend la forme d'un cône appelé cône pileux qui coiffe l'ébauche de la fibre.

I a formation de la fibre est précédée de l'apparition du muscle arrecteur, du même côté que les glandes sudoripares et sébacées, qui relie la gaine fibreuse du follicule à la base de l'épiderme.

\section{c) Croissance et éruption de la fibre.}

Le cone pileux et la fibre qu'il renferme croissent selon l'axe du follicule en se dirigeant vers l'épiderme. Au niveau des glandes sébacées,

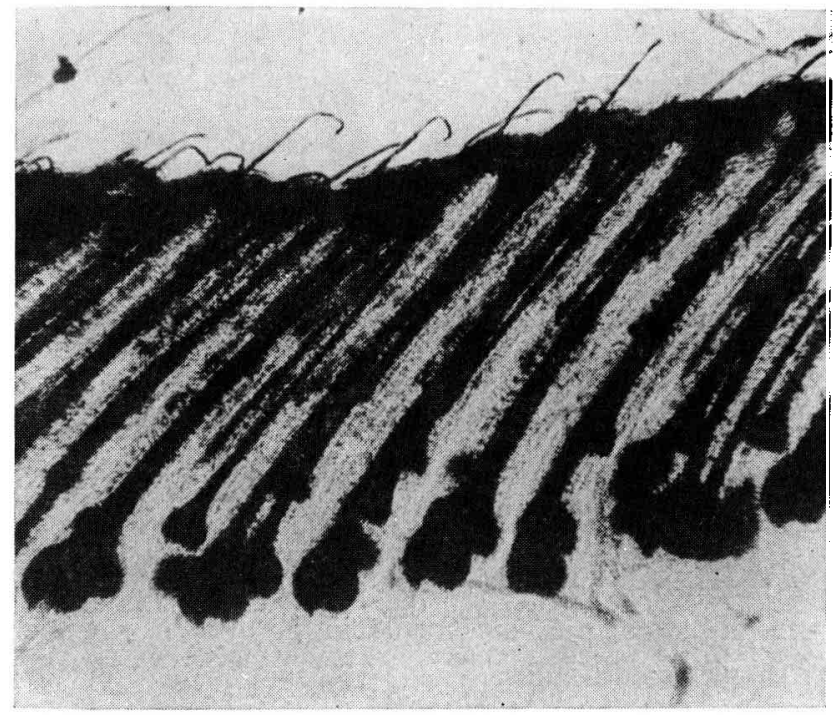

FIG. 7 ter. - Fœtus Karakul de I ro jours (dos). Remarquer le mode d'íruption des poils à la surface de la peau et le début d'incurvation des fibres.

la fibre traverse le sommet du cône pileux, la gaine interne arrêtant sa croissance à ce niveau. L a fibre s'engage alors dans le canal pileux, espace qui s'est formé par la dégénérescence des cellules axiales du follicule et qui la conduit jusqu'à l'intérieur des couches épidermiques où elle reste bloquée. La partie supérieure de la fibre s'incurve sous l'influence de la poussée de la matrice et finit par rompre l'épiderme pour arriver à la surface de la peau, l'extrémité restant toujours bloquée. Ce n'est qu'ultérieurement que le sommet de la fibre arrivera à se dégager et à apparaître à la surface de la peau (fig. 7 ter). 
Au début de leur éruption les poils de la région ventrale sont plus développés que ceux du flanc et du dos. Par la suite, la croissance des poils se ralentit sur le ventre et s'accélère sur le flanc et sur le dos. La figure 8 montre les différents gradients du développement des poils à la surface de la peau et leurs modifications avec l'âge.
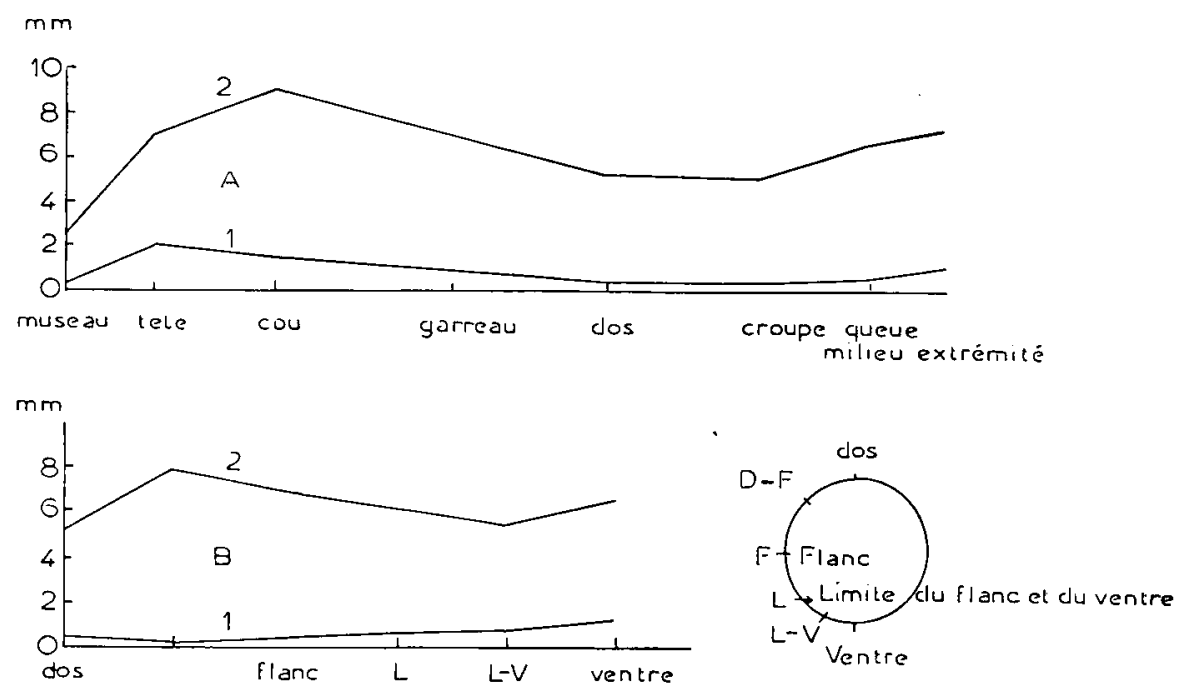

FIG. 8 .- Gradient de la longueur des poils chez le foetus Karakul. A : gradient antéro-postérieur. $-\mathrm{B}$ : gradient dorso ventral. - I : embryon Karakul de i ro jours. -2 : embryon Karakul de $\mathrm{r}_{32}$ jours. $-\mathrm{L}$ : ligne latérale $=$ limite du flanc et du ventre caractérisée par le changement d'orientation des follicules.

\section{$3^{\circ}$ Chronologie de l'évolution des follieules chez l'embryon Karakul et constitution des groupes folliculaires.}

Le mode d'apparition des follicules chez la race Karakul est le même que chez toutes les races ovines. On distingue chez le fœtus trois phases dans l'apparition des follicules.

Le développement folliculaire commence par l'apparition des premiers follicules isolés répartis irrégulièrement à la surface de la peau. Cette phase du développement est appelée phase "pré-triade". Elle est suivie de la phase " triade " dans laquelle de nouveaux follicules appelés $x$ apparaissent de chaque côté des $\mathrm{X}$ pour former des groupes de trois, d'où le terme " triade ». Leur emplacement est bien défini de sorte que sur une coupe parallèle à la surface de la peau ils forment chacun un angle d'environ $100^{\circ}$ avec $\mathrm{X}$ et sa glande sudoripare. Du fait de leur apparition relativement précoce, les follicules $\mathrm{X}$ et $x$ sont appelés follicules primaires. Ils possèdent toujours des glandes sébacées, sudoripare et un muscle arrecteur. Le follicule $\mathrm{X}$ est appelé membre central de la " triade " et les $x$, membres latéraux (fig. I6). 
Plus tard de nouveaux follicules font leur apparition et se placent près des follicules primaires et du côté opposé à leurs glandes sudoripares. Ce sont des follicules secondaires, caractérisés par l'absence de glande

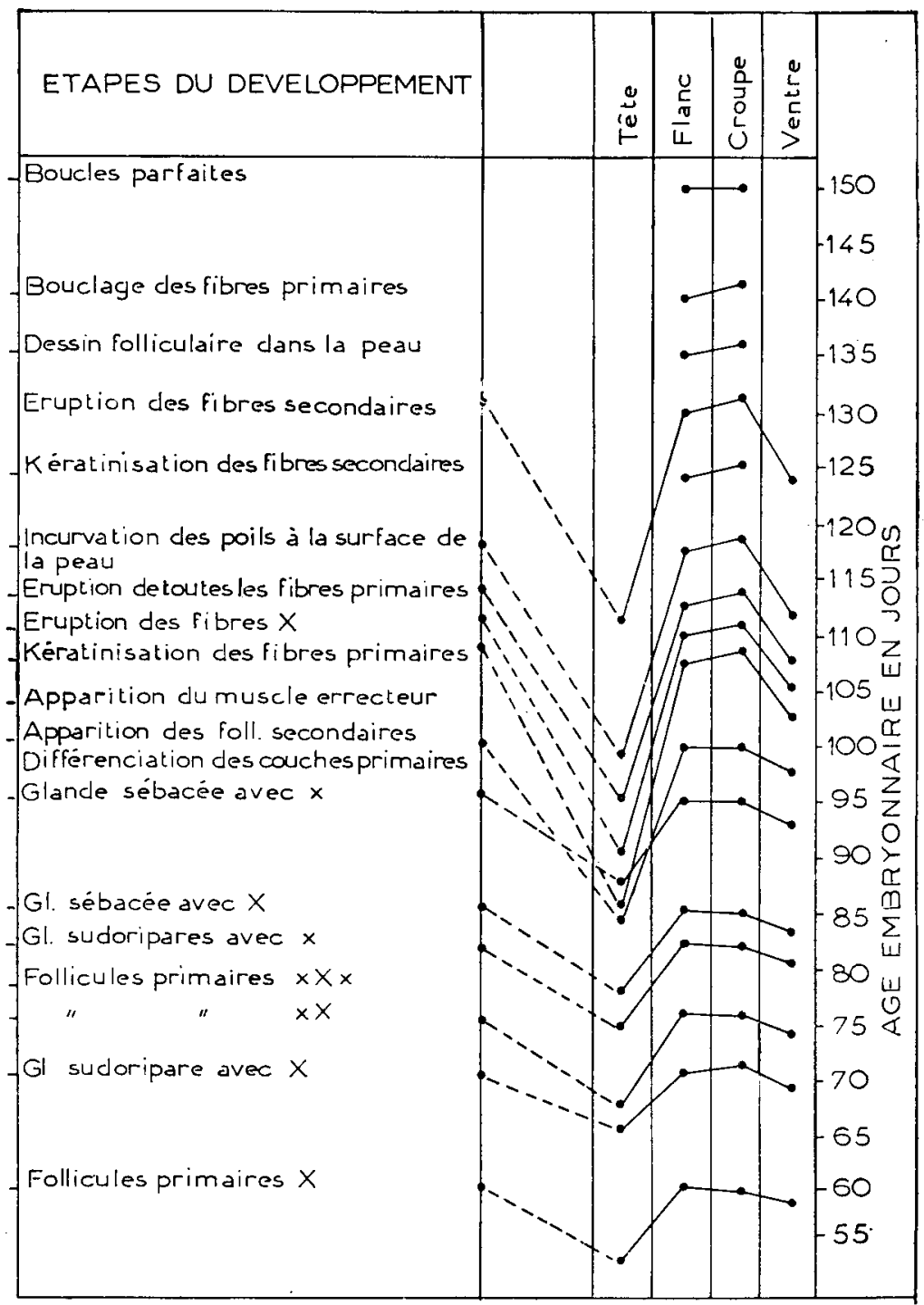

FIG, 9. - Étapes du développement folliculaire chez l'embryon Karakul en fonction de l'âge et sur les différentes parties du corps.

sudoripare. L'apparition des follicules secondaires caractérise la phase " post-triade » du développement folliculaire.

Les follicules pileux qui entourent les yeux, les narines, et la bouche, sont beaucoup plus précoces et plus volumineux que ceux qui recouvrent 
la surface du corps; notons que l'on ne considère pas les fibres qui en sont issues comme appartenant à la toison. Ils se forment avant le $45^{\mathrm{e}}$ jour de la vie fotale probablement vers le $40^{\mathrm{e}}$ jour.

Sur la surface du corps, les follicules pileux apparaissent et se différencient dans l'ordre qui suit. Il est évident que chaque partie du corps présente successivement les 3 phases du développement folliculaire mais à des dates différentes. I a figure 9 et le tableau VIII permettent de suivrele développement folliculaire sur les différentes parties de la surface du corps.

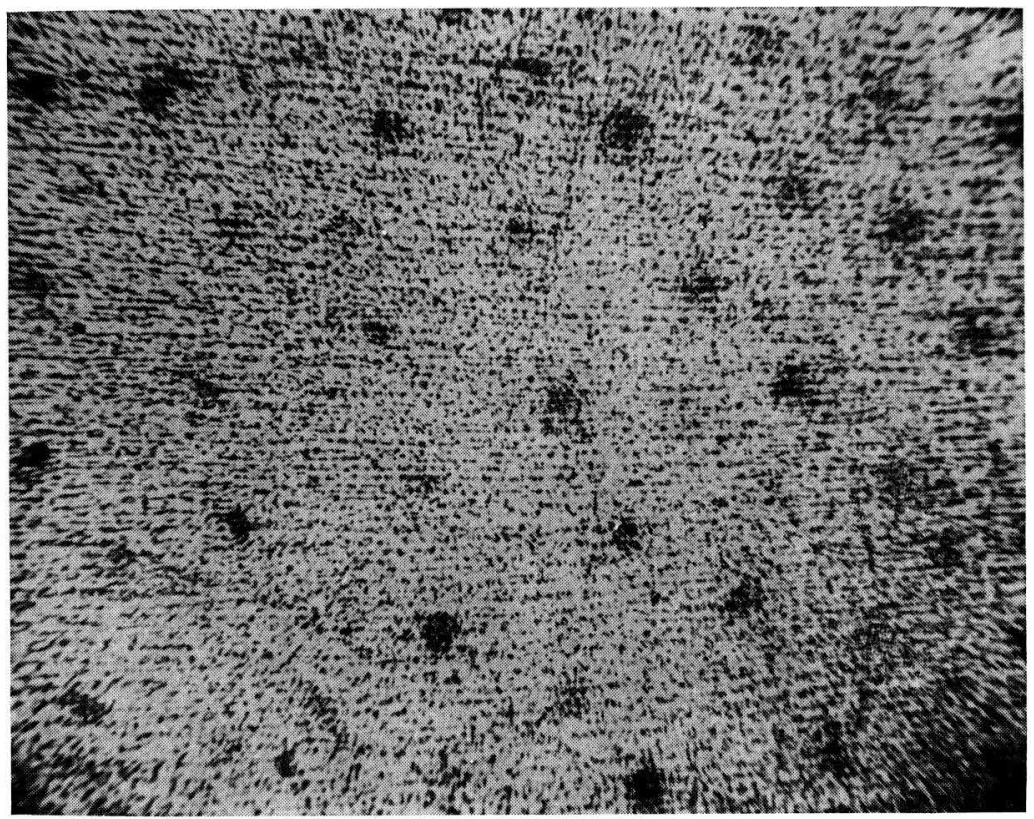

Fig. io. - - Embryon Karakul de 56 jours (tête). Les premiers grermes de follicules primaires X sont déjà formés à raison de $20 / \mathrm{mm}^{2}$ (vue par transparence ajurés coloration en masse).

\section{a) Phase pré-triade.}

Cette phase commence dès le $50^{\mathrm{e}}$ à $55^{\mathrm{e}}$ jour de la vie fotale sur la tête et l'avant-bras, de sorte qu'au $5^{6^{\mathrm{e}}}$ jour, on compte sur la tête une densité folliculaire égale à $20 / \mathrm{mm}^{2}$. A cet âge, les follicules ne sont pas encore visibles sur des coupes parallèles à la surface de la peau. I Leur mise en évidence ne se fait que sur des coupes perpendiculaires ou encore par l'examen de lambeaux de peau, après une coloration en masse suivie d'un éclaircissage au salicylate de méthyle (fig. Io).

$\mathrm{Au} 62^{\mathrm{e}}$ jour, les follicules $\mathrm{X}$ ont déjà envahi toute la surface du corps, avec, toutefois, une densité plus faible sur le flanc et sur la croupe. Par conséquent, sur le dos et le ventre, les germes de follicules doivent apparaître avant le $62^{\mathrm{e}}$ jour, soit aux environs du 59 à $60^{\mathrm{e}}$ jour. 
Ainsi on constate que les différentes phases du développement folliculaire sont plus précoces sur la tête, viennent ensuite successivement les membres, la queue, le ventre et le dos, et enfin le flanc et la croupe.

La différenciation des premières glandes sudoripares commence peu avant le $68^{\mathrm{e}}$ jour sur la tête. Au $68^{\mathrm{e}}$ jour, elles sont déjà formées sur toute la surface du corps sauf sur la croupe. A cet âge, c'est la tête qui est la plus riche en follicules avec $60 / \mathrm{mm}^{2}$, viennent ensuite le ventre $\left(38 / \mathrm{mm}^{2}\right)$, le dos, le flanc $\left(28 / \mathrm{mm}^{2}\right)$ et la croupe $\left(22 / \mathrm{mm}^{2}\right)$.

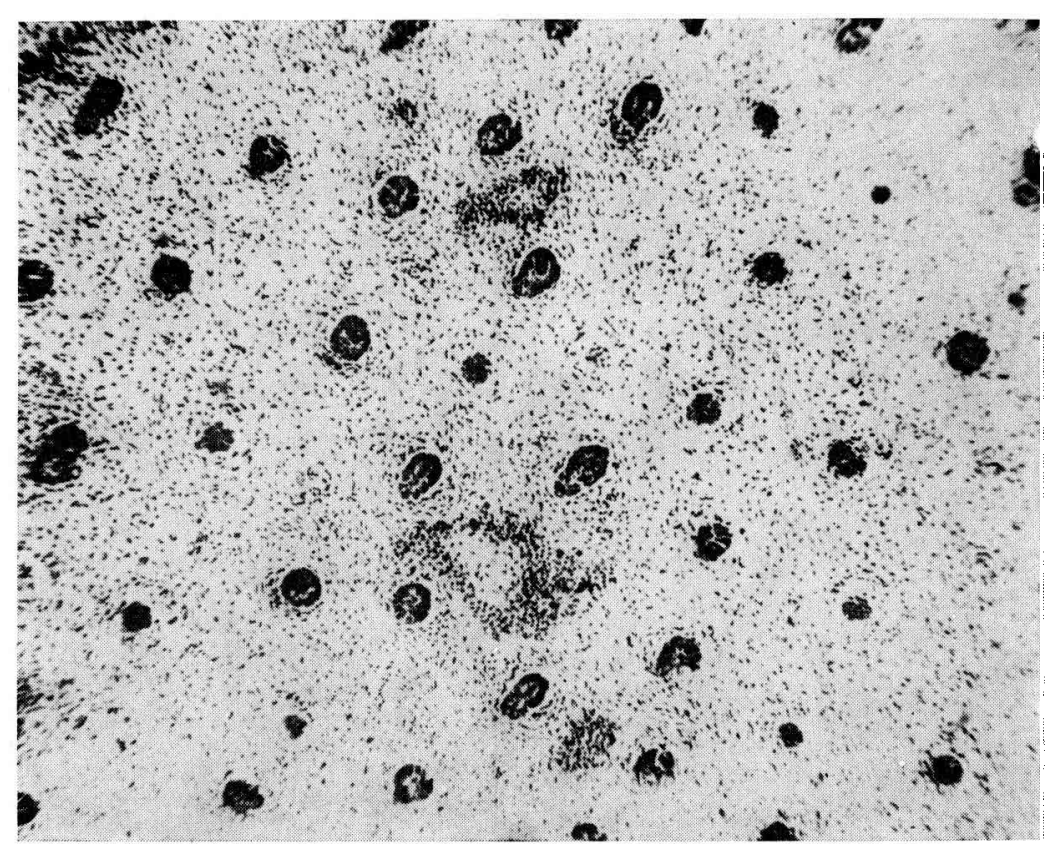

FIG. II. - Fœtus Karakul de 75 jours (flanc). Coupe transversale de la peau montrant les follicules primaires $\mathrm{X}$ et leurs glandes sudoripares.

\section{b) Phase triade.}

Toujours au $68 \mathrm{e}$ jour, mais sur la tête seulement, il y a formation d'un élément nouveau: l'ébauche des follicules primaires latéraux $(x)$ qui se placent de part et d'autre des X, leurs emplacements étant bien définis comme nous l'avons indiqué précédemment.

La phase triade n'est vraiment complète sur la tête que peu avant le $75^{\mathrm{e}}$ jour de la vie fœtale et dure environ une douzaine de jours sur cette même région (fig. I2). Au $75^{\mathrm{e}}$ jour, la triade est seulement ébauchée sur le cou, le ventre et le dos, mais n'existe pas encore sur la croupe et sur le flanc (fig. II).

Peu avant $1 \mathrm{e} 85^{\mathrm{e}}$ jour, les glandes sudoripares des follicules primaires latéraux $(x)$ sont différenciées sur toute la surface du corps. 
Les premières glandes sébacées font leur apparition entre le $75^{\mathrm{e}}$ et le $85^{\mathrm{e}}$ jour, d'abord sur la tête, puis sur le ventre, le flanc et la croupe (fig. I3 et I3 bis).

Quoique la disposition des follicules primaires par groupe de trois soit une règle générale, les exceptions ne manquent pas. En effet il n'est pas rare de constater un follicule $x$ qui paraît commun à deux triades. Il arrive parfois aussi qu' un follicule $X$ reste solitaire sans être accompagné de $x$.

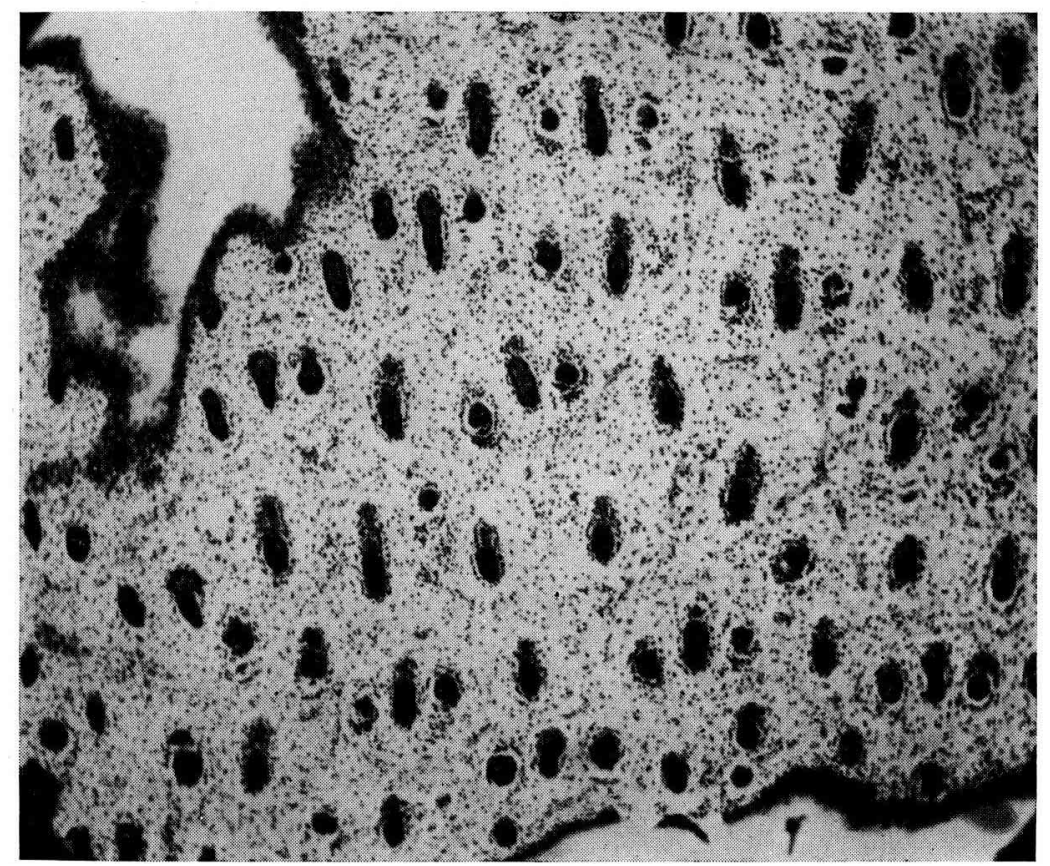

FIG. I2. - Fotus Karakul de 75 jours (tête). Remarquer la disposition des $x$ de chaque côté des $\mathrm{X}$.

\section{c) Phase post-triade.}

Caractérisée par l'apparition des follicules secondaires, cette phase commence peu avant le $85^{\mathrm{e}}$ jour sur la tête et au $97^{\mathrm{e}}$ jour sur le ventre. Elle n'apparaitra sur le flanc et la croupe que quelques jours après. Les fibres issues des follicules secondaires se différencient avant le $I I^{\mathrm{e}}$ jour sur la tête, au Ir $5^{\mathrm{e}}$ jour sur le ventre et au I2 $\mathrm{I}^{\mathrm{e}}$ jour sur le flanc et la croupe; leur éruption à la surface de la peau n'a lieu qu'entre le rio et le $I r 5^{\mathrm{e}}$ jour sur la tête, peu avant le $\mathrm{I} 32^{\mathrm{e}}$ jour sur le ventre et au $\mathrm{I}_{3} 2^{\mathrm{e}}$ jour sur le flanc et la croupe. La région ventrale compte beaucoup moins de follicules secondaires que le flanc et la croupe. La tête en est souvent dépourvue. 


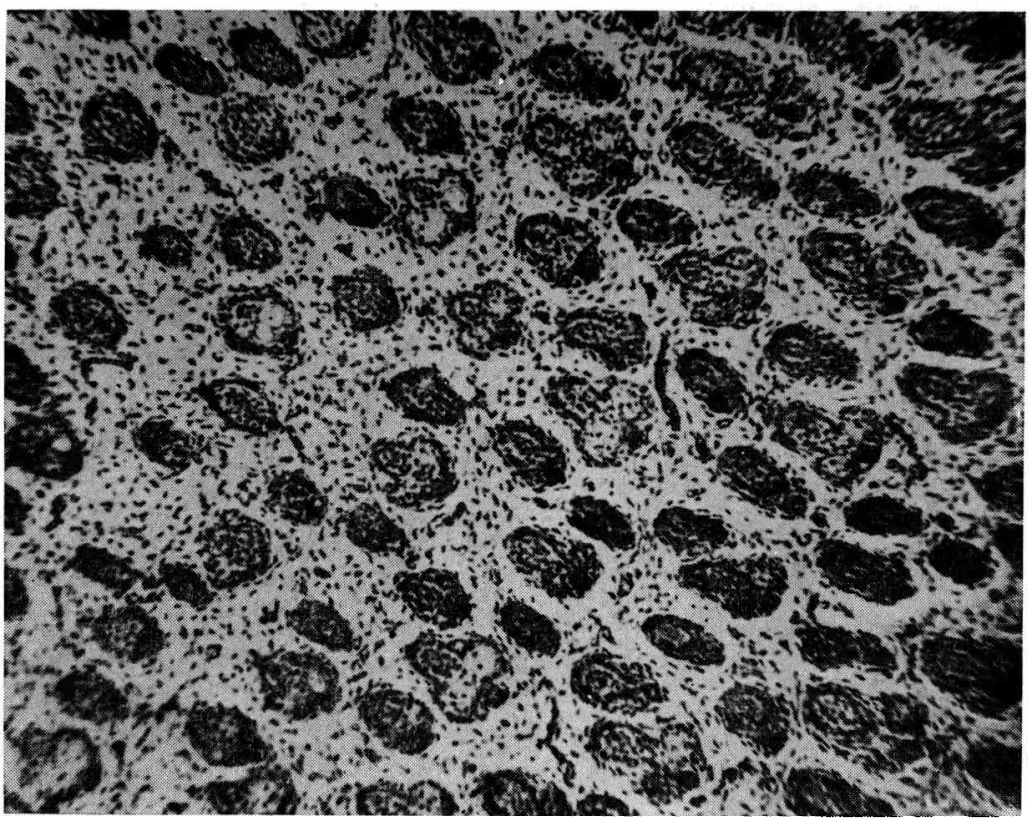

FIC, I3. - Fotus Karakul de 85 jours (flanc). Remarquer la précocité des membres centraux des triades.

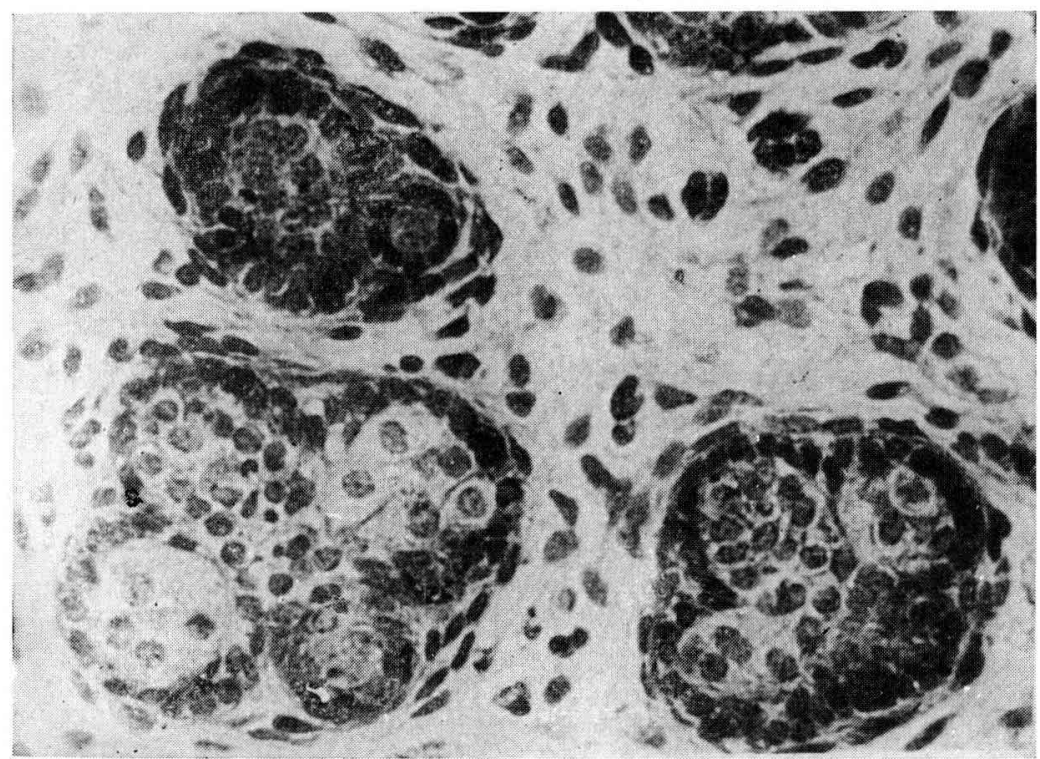

Fic. I 3 bis. - Fotus Karakul de 85 jours (flanc), montrant le détail de 2 follicules centraux $\mathrm{X}$ et d'un follicule latéral $x$ dont la slande sébacée n'est pas encore différenciée. 
Quant aux fibres primaires, seules les fibres issues des follicules $\mathrm{X}$ sont sorties à la surface de la peau au $97^{\mathrm{e}}$ jour sur la tête, peu avant le $\mathrm{IIO}^{\mathrm{e}}$ jour sur le ventre, la croupe et le flanc. Au $I x 5^{\mathrm{e}}$ jour, toutes les fibres primaires sont sorties à la surface du corps. Les différents gradients $\mathrm{du}$ développement folliculaire sont visibles extérieurement grâce à cette éruption des fibres (fig. 8). L'existence de ces gradients montre que pour une même région du corps, le flanc par exemple, certaines parties sont plus avancées que d'autres au point de vue du développement folliculaire.

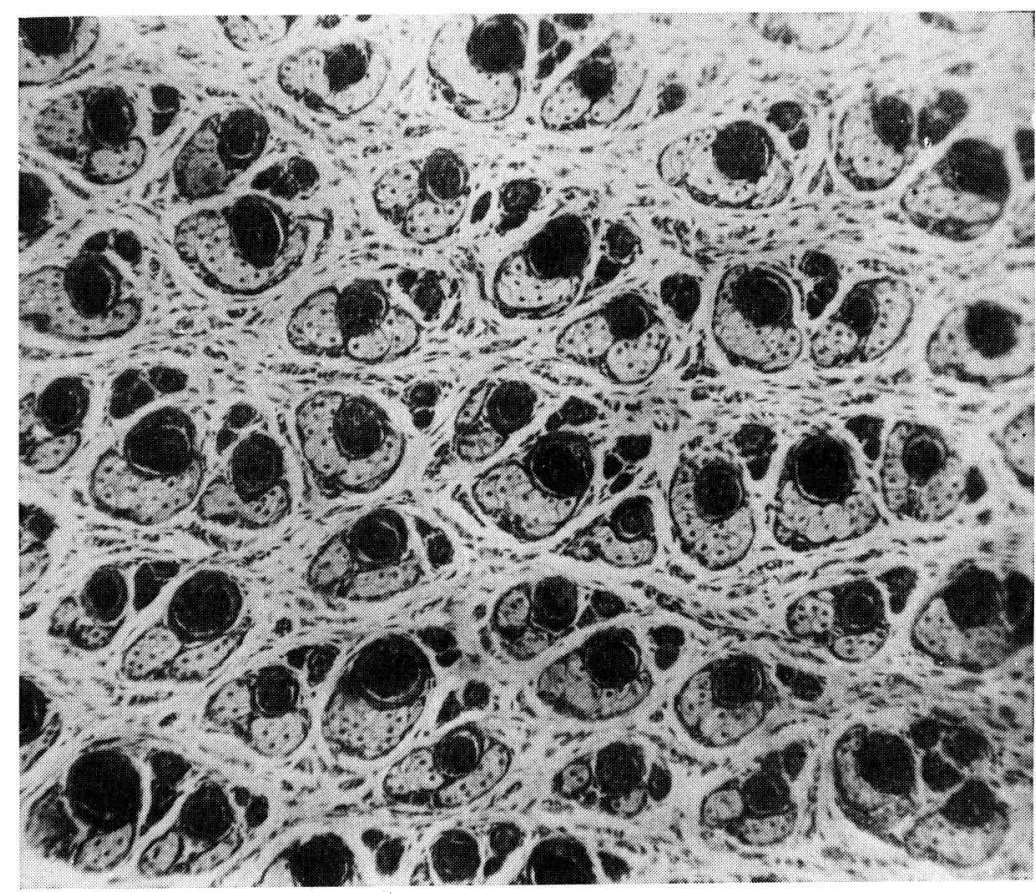

FIG. 14. - Fœtus Karakul de I2I jours (flanc). Coupe transversale des follicules au niveau des glandes sébacées.

La différenciation du muscle arrecteur des follicules primaires a lieu, pour chaque région du corps, après l'apparition des follicules secondaires mais avant la kératinisation des fibres primaires.

Chez le fœtus de IzI jours, dont les poils sont déjà sortis à la surface de la peau, les follicules sont disposés en groupes séparés par des trames de tissu conjonctif (fig. I4). Cette disposition va en s'accentuant de la base au sommet de sorte qu'au niveau des bulbes les follicules sont sans aucun ordre. Cette disposition reste inchangée chez l'agneau (fig.I7).

La profondeur des follicules primaires dans la peau (distance de la base du bulbe à la surface de la peau) est de $1,05 \mathrm{~mm}$. 
Les follicules ainsi que leurs fibres ont une forme courbe. Cette incurvation a lieu après la kératinisation de la fibre. Les extrémités des poils sortis de la peau sont dirigées vers la surface de celle-ci (fig. I5).

A ce stade du développement fœtal, la peau est déjà utilisable, sous la nomination "galyac " ou "lessac".

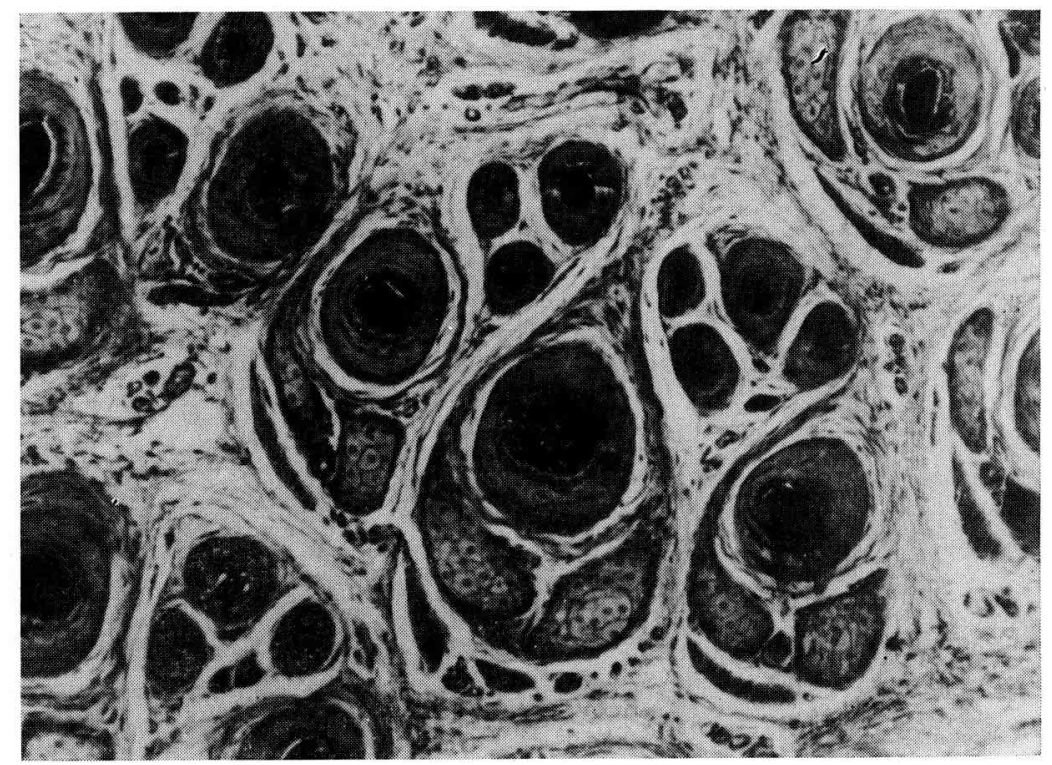

FIG. I 5. - Agneau Karakul à la naissance.

Remarquer la disposition des différents follicules à l'intérieur de la triade.

Au $\mathrm{I}_{32^{\mathrm{e}}}$ jour, les membres des groupes folliculaires sont presque au complet. I es fibres primaires étant déjà sorties de 4 à $7 \mathrm{~mm}$ à la surface de la peau, montrent la forme définitive de leur section transversale. Celle-ci est circulaire près du sommet et variable quand on s'en éloigne. Les follicules primaires peuvent atteindre $I, 3 \mathrm{~mm}$ de profondeur et forment un angle variant de $40-80^{\circ}$ avec la surface de la peau. Le niveau des bulbes des follicules secondaires se situe à mi-hauteur des follicules primaires.

Durant la dernière moitié du $5^{\mathrm{e}}$ mois de la vie foetale, peu de changements sont observés dans le développement des follicules primaires. Par contre les follicules secondaires continuent leur développement et leur différenciation. Le nombre de fibres secondaires continue à augmenter même après la naissance de l'agneau.

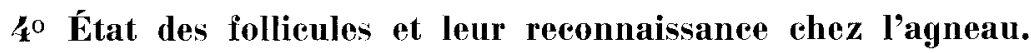

A la naissance de l'agneau, les groupes folliculaires sont définitivement formés ; sur une coupe parallèle à la surface de la peau on observe 


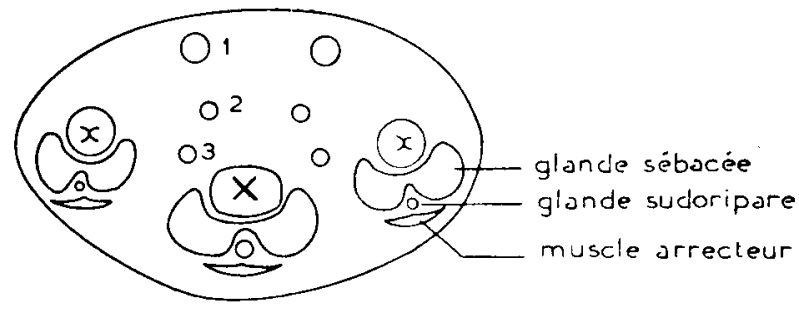

Fig. т6. - Schéma d'une triade folliculaire

avec les follicules secondaires correspondants numérotés par ordre de leur apparition.

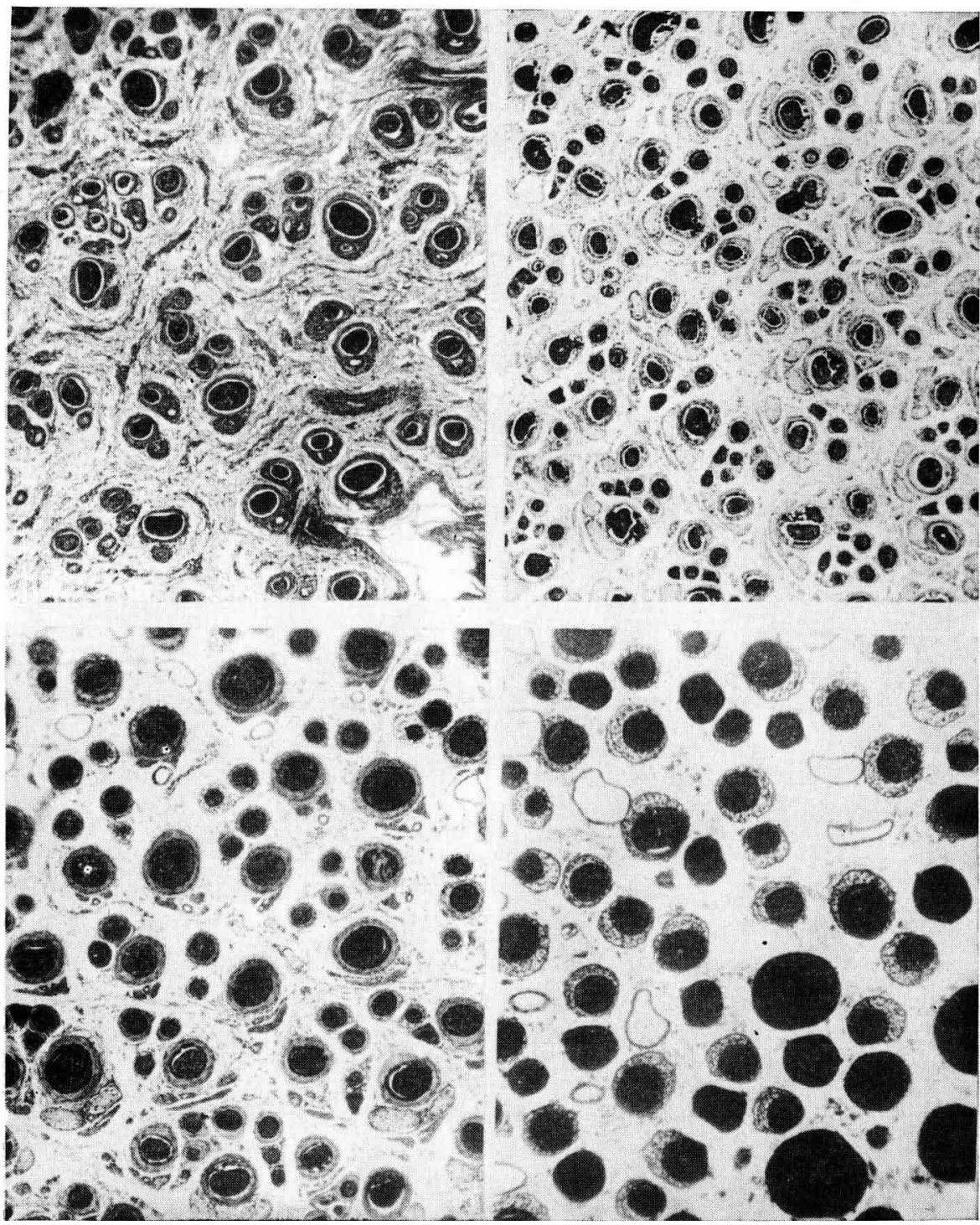

FIG. I7. - Coupe transversale des follicules à différentes profondeurs chez un agneau Karakul à la naissance $: a$ ) près de la surface ; $b$ ) au niveau des glandes sébacées ; $c$ ) en dessous des glandes sébacées; $d$ ) au niveau des bulbes. 
au niveau des glandes sébacées, le schéma classique des groupes folliculaires (fig.I6), appelés "( triades 》 accompagnés de leurs follicules secondaires. I a disposition par triade n'est nettement visible qu'au niveau des glandes sébacées. Il est plus difficile de la reconnaitre quand on s'éloigne de part et d'autre de ce niveau (fig. I7).

On peut reconnaittre les différentes catégories de follicules chez l'agneau : les premiers follicules formés $(\mathrm{X})$ sont disposés irrégulièrement dans la peau. On les reconnait facilement du fait qu'ils sont les membres centraux des triades (fig. I5).

Chez la race Karaku1, ils diffèrent des membres latéraux appelés $x$, par leur taille plus grande, leur développement et différenciation plus avancée et leur pigmentation plus intense. Lorsqu'il y a formation de poil hétérotypique, celui-ci est presque toujours produit par les follicules primaires centraux.

Quant aux follicules secondaires, ils sont de petite taille et implantés moins profondément que les primaires. Plus ils sont anciens, plus ils sont éloignés des primaires. Ils sont disposés comme nous l'avons vu, entre $\mathrm{X}$ et $x$ et du côté opposé au muscle arrecteur. Les follicules secondaires les premiers formés sont différenciés et les derniers formés sont encore à l'état de germes non différenciés chez l'agneau à la naissance. Ils sont souvent dépourvus de glandes sébacées; lorsqu'elles existent, elles sont situées plus près de la surface de la peau que celles des primaires. Rappelons que les follicules secondaires n'ont jamais de glande sudoripare et c'est là un caractère distinctif.

\section{C. - CARACTÉRISTIQUES DES FIBRES CHEZ LE FGETUS ET L'AGNEAU}

\section{$1^{0}$ Toison de l'agneau à la naissance :}

\section{a) Différentes sortes de fibres.}

La toison de l'agneau Karakul est constituée essentiellement par deux sortes de fibres : des fibres longues et grossières, les poils, et des fibres courtes et fines, les brins de laine.

D'ailleurs d'autres caractères que les dimensions différencient ces deux sortes de fibres : le poil présente une cuticule composée d'écailles de forme plus ou moins hexagonale, tandis que chez le brin de laine les écailles sont coronales ou subcoronales, selon qu'elles font le tour entier de la fibre ou au moins la $\mathrm{I} / 2$ du tour (fig. I8). Le poil présente un canal médullaire de dimension et de forme très variables : le diamètre de ce canal peut atteindre les $2 / 3$ de celui de la fibre. I1 peut être continu sur 
toute la longueur de la fibre ou présenter de courtes interruptions (canal médullaire fragmentaire). Quelquefois, même, la moelle ne forme plus du tout de canal et ne se présente que comme des îlots à intervalles éloignés dans l'axe de la fibre.

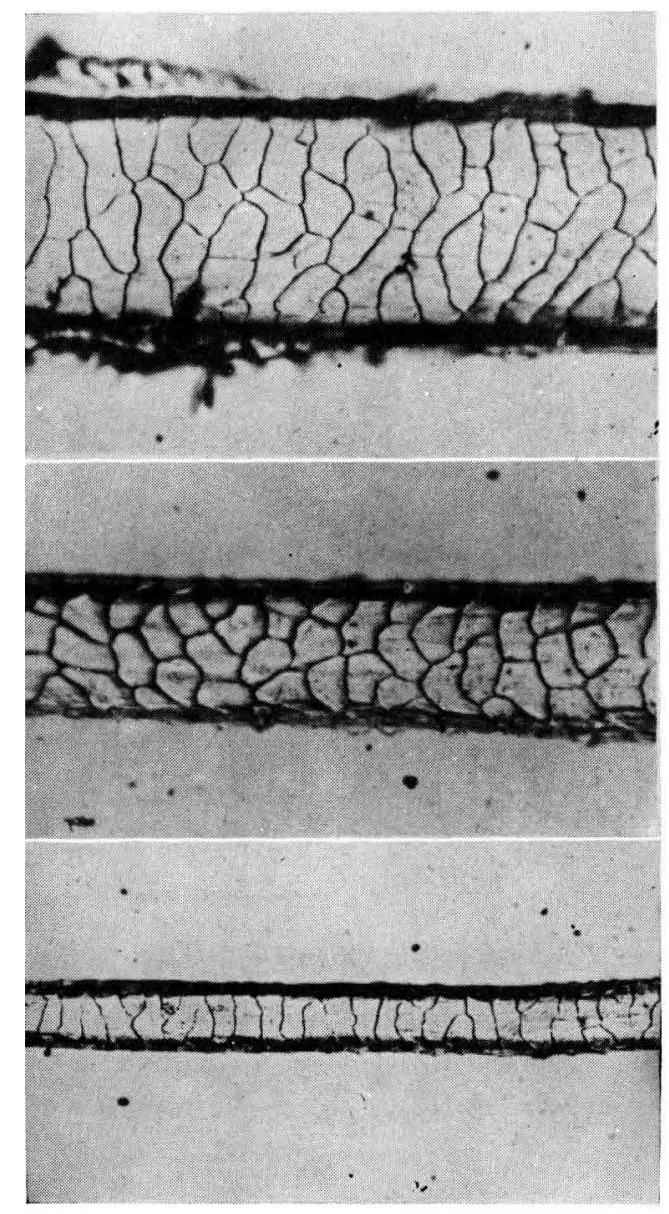

FIG. I8. - Empreintes des écailles de la cuticule des fibres chez l'agneau Karakul. - En haut : poil hétérotypique, - Au milieu : poil. - En bas : laine (duvet).

Par contre, chez la laine, l'absence de moelle est caractéristique.

La section transversale est plus ou moins circulaire pour les brins de laine et circulaire ou polygonale pour le poil.

Il arrive cependant quelquefois, chez le Karakul, que le poil commence à pousser sous la forme de jarre : écailles rectangulaires de la cuticule, canal médullaire énorme, dont le diamètre atteint les 9/ro de celui de la fibre, section transversale ovale mais très aplatie. Cette structure jarreuse se développe sur les 2-3 premiers centimètres de la fibre ; par 
la suite, celle-ci croît avec la structure caractéristique du poil. Comme chez l'adulte, cette dernière structure constitue de loin la plus grande partie de ces fibres, nous les qualifierons de "poils hétérotypiques " par opposition aux hétérotypes proprement dits qui présentent sur leur partie proximale la structure de la laine.

Chez le Karakul, dans la majorité des cas, les poils sont produits par les follicules primaires et lorsque l'agneau présente dans sa toison des poils hétérotypiques ceux-ci sont produits par le follicule primaire central. Par contre la laine est une production caractéristique des follicules secondaires. Il arrive cependant que les follicules secondaires, en particulier les follicules secondaires marginaux, produisent des fibres assez grossières présentant une légère médullation : ces fibres mal définies sont intermédiaires, dans leur structure, entre le poil et la laine.

Ainsi, la toison de l'agneau Karakul est assez voisine de celle du type sauvage : pelage composé de deux parties : partie externe formant le pelage de protection, composé des poils grossiers provenant des follicules primaires et partie formant le pelage d'isolement thermique, composé de fines fibres de laine équivalentes au duvet.

\section{b) Rapport $\mathbf{S} / \mathbf{P}$.}

Les rapports $\mathrm{S} / \mathrm{P}$ folliculaires $(\mathrm{S} / \mathrm{PI}$ et $\mathrm{S} / \mathrm{P} 2)$ sont différents d'un individu à l'autre et chez le même animal, ils varient avec la région du corps.

\section{TABLEAU IV}

Valeur du rapport S/P 2 chez différents foetus, agneaux et adultes Karakul, suivant leur âge (en jours).

\begin{tabular}{|c|c|c|c|c|c|c|c|c|c|c|c|c|}
\hline \multirow{2}{*}{ Animaux } & \multirow[t]{2}{*}{ Age } & \multicolumn{4}{|c|}{ Fœtus (F) } & \multicolumn{6}{|c|}{ Agneaux (A) } & \multirow{2}{*}{$\begin{array}{l}\text { Adultes } \\
\text { (Ad) }\end{array}$} \\
\hline & & I IO & $\mathrm{I} 2 \mathrm{I}$ & ${ }^{1} 3^{2}$ & $\mathbf{r}_{42}^{2}$ & I & $3^{\circ}$ & 60 & 100 & I80 & $3^{6} 5$ & \\
\hline$F$ & $\begin{array}{l}38 \\
29 \\
\mathbf{2 8} \\
1 \mathbf{2}\end{array}$ & ० & $0, \mathrm{I}$ & 0,6 & $\mathrm{I}, 5$ & & & & & & & \\
\hline $\mathrm{A}$ & $\begin{array}{l}x \\
20 \\
22 \\
23 \\
24 \\
26 \\
3 \mathrm{I} \\
32 \\
35\end{array}$ & & & & & $\begin{array}{l}2,00 \\
2,00 \\
2,50 \\
2,60 \\
2,00 \\
1,21 \\
2,50 \\
1,23\end{array}$ & $\begin{array}{l}2,80 \\
3,00 \\
2,68 \\
2,23 \\
\mathrm{I}, 66\end{array}$ & $\begin{array}{l}3,00 \\
3,5^{\circ}\end{array}$ & $\begin{array}{l}3,40 \\
3,30 \\
2,86 \\
1,83 \\
3,30 \\
3,00\end{array}$ & $\begin{array}{l}3,20 \\
3,00 \\
2,50 \\
2,00\end{array}$ & $\begin{array}{l}3,26 \\
2,50 \\
2,40 \\
\\
1,42\end{array}$ & \\
\hline Ad & $\begin{array}{c}? \\
9 \\
660\end{array}$ & & & & & & & & & & & $\begin{array}{l}3 \\
2 \\
3\end{array}$ \\
\hline
\end{tabular}


Sur la tête et les membres, il y a peu ou pas de follicules secondaires et par suite, les rapports $\mathrm{S} / \mathrm{P}$ folliculaires sont voisins de zéro. Par contre sur le dos et le flanc, le rapport $\mathrm{S} / \mathrm{P} 2$ est plus élevé et peut atteindre 2,5 . Sa valeur normale est de 2 chez l'agneau Karakul à la naissance (tableau IV) (8 agneaux contrôlés). L re rapport $\mathrm{S} / \mathrm{P}$ I est, naturellement, plus élevé que les valeurs ci-dessus.

\section{c) Diamètre des fibres.}

Io ooo mesures de diamètre ont été effectuées sur 8 agneaux à différents âges ( $\mathrm{I}$ jour, I mois, 3 mois, 6 mois et $I$ an). Les résultats des mensurations ont été représentés par une courbe de fréquence pour chaque échantillon. Étant donné l'existence de différences individuelles notables, nous avons pris comme type deux agneaux les plus caractéristiques, $1^{\prime}$ un à poils médullés $\left(\mathrm{n}^{0} 23\right)$ et 1'autre à poils non médullés $\left(\mathrm{n}^{0} 22\right)$.

a) Cas des agneaux à poils médullés : Les mesures de diamètre ont été réparties sur un histogramme ayant Io $\mu$ comme intervalle de classe. Il s'agit d'un histogramme polymodal représentant un mélange de trois populations : les fibres primaires $\mathrm{X}$, les fibres primaires $x$ et enfin les fibres secondaires (fig. I).

L'un des modes oscille autour de $20 \mu$ et correspond indiscutablement aux fibres secondaires, comme le montre l'étude morphologique des fibres. Les deux autres se situent à $40-45 \mu$ et $65-80 \mu$. On peut les interpréter comme représentant les fibres issues des follicules primaires $\mathrm{X}$ pour les plus grosses et des follicules primaires $x$ pour les moins grosses.

ß) Cas des agneaux à poils peu médullés : Les deux histogrammes du diamètre des fibres chez 1'agneau $n^{0} 22$ à la naissance, construits selon la méthode des coupes histologiques et la méthode de W. I. R. A. (fig.I), montrent en principe deux modes : celui des fibres secondaires autour de $20 \mu$ et celui des fibres primaires autour de $40 \mu$, la différence entre les $\mathrm{X}$ et les $x$ étant peu prononcée. En général ces agneaux ne présentent pas de poils hétérotypiques qui sont plus nombreux chez les agneaux à poils médullés. Or les poils hétérotypiques sont issus des follicules $\mathrm{X}$, ce qui explique que l'histogramme des diamètres présentent trois modes chez les agneaux à poils médullés et deux modes chez les agneaux à poils non médullés.

\section{d) Longueur des fibres.}

Les longueurs des fibres ont été mesurées par notre méthode pour I7 peaux afghanes de différents types. Les résultats sont indiqués dans le tableau XIII du chapitre suivant. Les longueurs moyennes des fibres 
ont été calculées pour chaque individu et pour chaque catégorie de fibres. Les valeurs extrêmes (pour les moyennes) sont les suivantes :

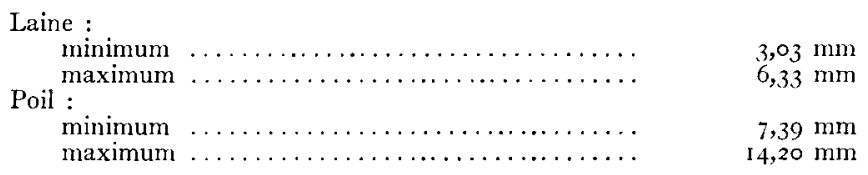

Ainsi la mesure des longueurs des fibres chez le jeune agneau permet une différenciation très nette des 2 catégories de fibres : poil et laine (fig. 60).

La longueur des poils, comme nous le verrons au chapitre III est en relation avec les caractéristiques de la boucle.

\section{Modifications avec l'âge.}

Les différentes caractéristiques de la toison évoluent depuis le stade fœtal jusqu'au stade adulte. Ces modifications portent notamment sur

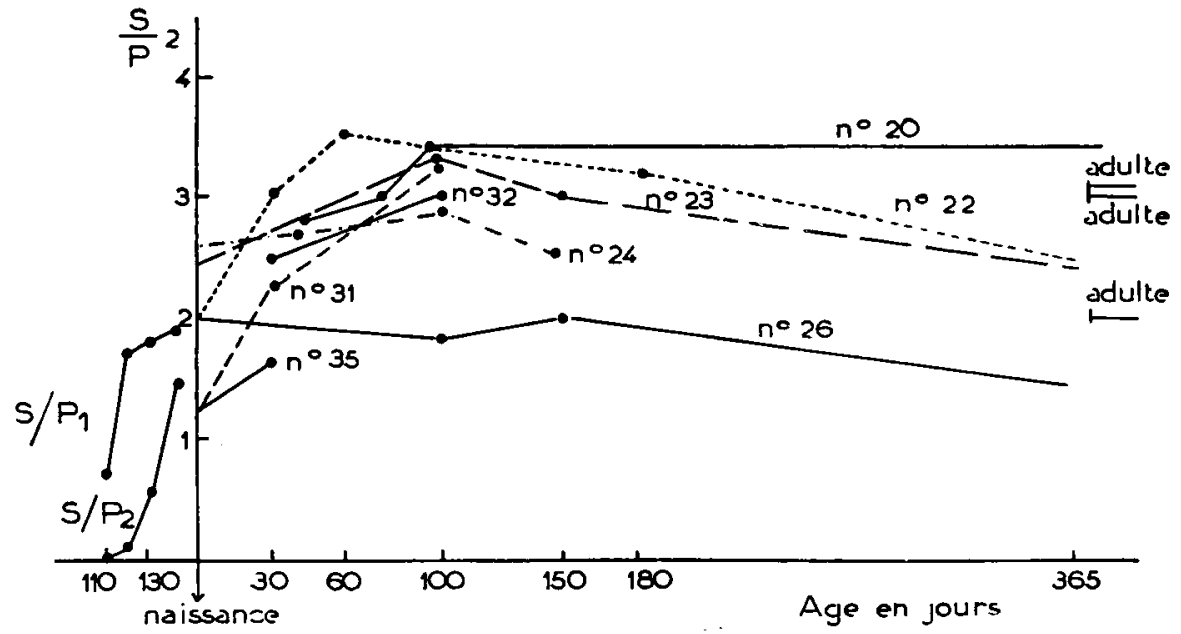

FIG. 19. - Variation du rapport $\mathrm{S} / \mathrm{P}_{2}$ avec l'âge chez le foetus et l'agneau Karakul.

le rapport $\mathrm{S} / \mathrm{P}$, la densité pileuse, le diamètre et la longueur des fibres. Nous avons étudié ces caractéristiques de la fibre sur les mêmes individus à des âges successifs ou sur des individus différents quand il n'était pas possible de faire autrement (cas des fœtus).

\section{a) Rapport $\mathbf{S} / \mathbf{P}$.}

Nous avons choisi la région du flanc au niveau de la dernière côte pour prélever les échantillons. L,es valeurs obtenues sont indiquées sur le tableau IV et la figure Ig. Cette figure montre que chez l'embryon de 
I Io jours les fibres secondaires ne sont pas encore différenciées $(\mathrm{S} / \mathrm{P} 2=0)$ bien que les follicules secondaires existent déjà $(\mathrm{S} / \mathrm{P} \mathrm{I}=0,7)$.

Jusqu'au $\mathrm{I} 2 \mathrm{O}^{\mathrm{e}}$ jour de la vie fœtale le rapport $\mathrm{S} / \mathrm{P} 2$ augmente lentement puis s'accélère jusqu'à la naissance. Cette augmentation continue avec le même rythme durant le premier mois chez l'agneau, ce qui s'explique par la différenciation de nouvelles fibres secondaires durant cette période.

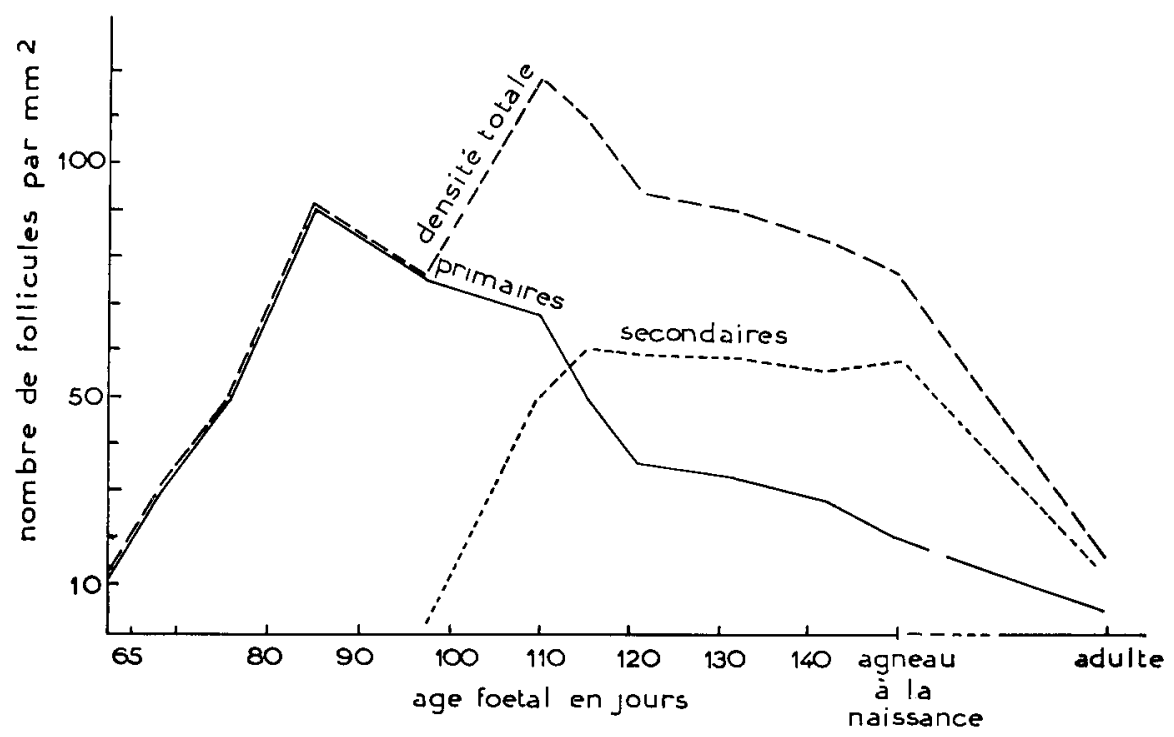

FIG. 20. - Variation avec l'âge de la densité folliculaire chez le fotus Karakul.

Alors qu'à la naissance il y a de nombreux germes folliculaires, chez les agneaux d'un mois nous n'en avons observé que très rarement. On pourrait donc en déduire que la formation de nouvelles ébauches folliculaires s'arrête après la naissance. Or le rapport $\mathrm{S} / \mathrm{P} 2$ augmente jusqu'au Ioo jour après la naissance, ce qui conduit à supposer que de nouveaux germes folliculaires peuvent apparaître pendant les deux premiers mois puisque le temps nécessaire à la formation d'une fibre à partir du moment où l'ébauche folliculaire est visible, est d'environ I mois.

Cette différence dans les conclusions tirées de l'examen histologique d'une part et de l'analyse graphique des variations de S/P 2 d'autre part, reste d'autant plus inexplicable que l'opinion des auteurs sur la genèse post-natale de nouveaux bourgeons folliculaires est très variable.

Après le Ioo $^{\mathrm{e}}$ jour le rapport $\mathrm{S} / \mathrm{P} 2$ reste constant. I1 semble, toutefois, se produire ultérieurement une légère diminution du rapport $\mathrm{S} / \mathrm{P} 2$. L'explication d'un tel phénomène, s'il existe, pourrait être donnée par la dégénérescence définitive de certains follicules secondaires, comme l'a supposé Burns (I949) chez les races Leicester et Romney. 


\section{b) Densité pileuse.}

Nous avons vu que le nombre total des fibres est en augmentation durant les tout premiers mois après la naissance. D'autre part, la surface

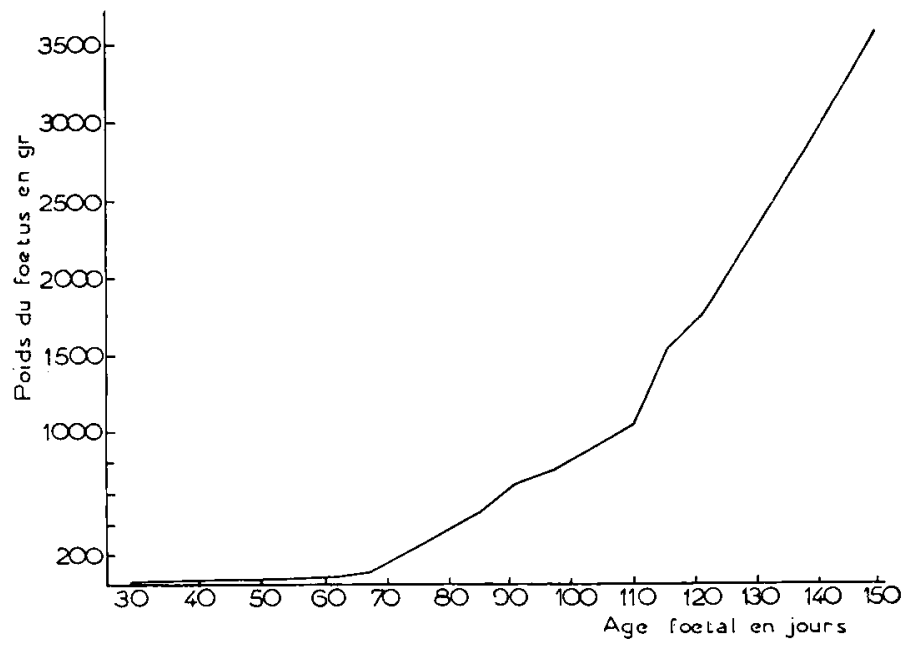

FIG. 2I. - Variation avec l'âge du poids du fœetus Karakul.

TABLEAU V

Variation avec l'âge de la densité folliculaire au niveau du flanc chez le foetus Karakul.

\begin{tabular}{|c|c|c|c|}
\hline \multirow{2}{*}{ Age fæatal } & \multicolumn{3}{|c|}{ Densité folliculaire } \\
\hline & Primaires & Secondaires & Densité absolue \\
\hline 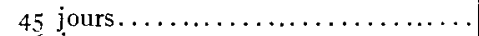 & $\circ$ & o & o \\
\hline $5^{6}$ jours $\ldots \ldots \ldots \ldots \ldots \ldots \ldots \ldots \ldots \ldots$ & 0 & 0 & o \\
\hline$\sigma_{2}$ jours $\ldots \ldots \ldots \ldots \ldots \ldots \ldots \ldots \ldots \ldots, \ldots \ldots \ldots$ & I I & 0 & I I \\
\hline 68 jours $\ldots \ldots \ldots \ldots \ldots \ldots \ldots \ldots \ldots$ & 28 & 0 & 28 \\
\hline 75 jours $\ldots \ldots \ldots \ldots \ldots \ldots \ldots \ldots \ldots \ldots$ & 50 & o & 50 \\
\hline 85 jours............................ & 90 & 0 & $9^{\circ}$ \\
\hline 97 jours $\ldots \ldots \ldots \ldots \ldots \ldots \ldots \ldots \ldots$ & 75 & 0 & 75 \\
\hline ino jours $\ldots \ldots \ldots \ldots \ldots \ldots \ldots \ldots \ldots$ & 68 & 50 & I 18 \\
\hline II 5 jours $\ldots \ldots \ldots \ldots \ldots \ldots \ldots \ldots \ldots$ & 50 & 60 & I 10 \\
\hline I 2 I jours $\ldots \ldots \ldots \ldots \ldots \ldots \ldots \ldots \ldots \ldots \ldots \ldots \ldots$ & 35 & 59 & 94 \\
\hline$r_{32}$ jours $\ldots \ldots \ldots \ldots \ldots \ldots \ldots \ldots \ldots$ & 32 & $5^{8}$ & 00 \\
\hline $\mathrm{I}_{42}{ }^{2}$ jours. $\ldots \ldots \ldots \ldots \ldots \ldots \ldots \ldots \ldots \ldots$ & 28 & $5^{6}$ & 84 \\
\hline Agneau à la naissance.............. & 19 & $5^{8}$ & 77 \\
\hline Adulte $\ldots \ldots \ldots \ldots \ldots \ldots \ldots$ & 4 & I 2 & I6 \\
\hline
\end{tabular}

de la peau augmente parallèlement. Il y a donc une relation inverse entre la densité pileuse totale et la croissance de l'agneau. Environ jusqu'au rroe jour de la vie fotale, l'augmentation rapide des follicules l'emporte sur 1a croissance de l'animal qui est relativement lente jusqu'à cet âge (tableau V, fig. 20 et $2 \mathrm{I}$ ), si bien que la densité pileuse croît. 
Puis c'est l'extension de la peau qui s'accélère, tandis que la formation de nouveaux follicules se ralentit : la densité pileuse décroît et cette chute de la densité pileuse continue jusqu'à l'âge adulte.

L'analyse de la figure 20 montre, comme on pouvait s'y attendre, des variations décalées dans le temps, de la densité des follicules primaires et de la densité des follicules secondaires. C'est ainsi que la densité des primaires augmente jusqu'au $85^{\mathrm{e}}$ jour de la vie foetale, puis décroît jusqu'à l'âge adulte, tandis que celle des secondaires croît jusqu'au II $5^{\text {e }}$ jour puis reste plus ou moins constante jusqu'à la naissance.

\section{c) Diamètre des fibres.}

a) Cas des individus à poils médullés: L'histogramme des diamètres, au $I{ }^{\prime} O^{\mathrm{e}}$ jour de la vie foetale, présente un seul mode (fig. 22). En effet seuls, à cet âge, les sommets des fibres primaires issus des follicules $\mathrm{X}$ sont sortis à la surface de la peau.

Au I2 $I^{e}$ jour on distingue deux modes, l'un correspondant aux follicules X et placé sur la droite de 1'histogramme, 1'autre correspondant aux follicules $x$ et placé sur la gauche (fig. 22). Ce dernier a une hauteur plus importante étant donné le nombre deux fois plus grand des $x$ par rapport aux X.

Plus tard, lorsque les fibres secondaires (duvet) se différencient, un troisième mode fait son apparition et se place à gauche des deux précédents. Sa hauteur augmente de plus en plus et il domine bientôt les deux autres. Ce phénomène se poursuit chez l'agneau (fig. I). On assiste également à une extension de 1'histogramme vers la droite; ceci est dû à l'augmentation moyenne du diamètre des poils et ce phénomène continue jusqu'à une vingtaine de jours après la naissance : le diamètre définitif d'une fibre est atteint à un niveau situé à $20 \mathrm{~mm}$ environ du sommet quand l'agneau est âgé d'environ 25 jours.

A partir du $3^{\mathrm{e}}$ et $4^{\mathrm{e}}$ mois, chez 1'agneau, l'histogramme présente une prédominance du mode correspondant aux fibres secondaires; les deux autres s'atténuent (fig. I). Toutefois, le mode relatif aux fibres les plus épaisses (les primaires) arrive toujours à se distinguer.

Chez l'animal adulte, cette courbe reste sensiblement la même avec peu de modifications dues à la chute et au remplacement périodique des fibres.

En observant la série des histogrammes ou des courbes de fréquence des diamètres des poils, on assiste, au cours de la première année à un tassement vers la gauche de l'histogramme et à une atténuation des différents modes en faveur d'un seul, celui qui correspond aux fibres fines. Ceci résulte d'une part de l'augmentation du nombre des fibres secondaires qui atteint son maximum vers le $3^{\mathrm{e}}$ mois, comme nous l'avons vu 
à propos du rapport $\mathrm{S} / \mathrm{P} 2$ et d'autre part, de l'affinement de la toison. Cet affinement est acquis à la suite d'une diminution avec 1'âge du diamètre moyen des poils qui est plus spectaculaire chez les animaux riches en poils hétérotypiques dont la partie distale est aplatie et épaisse alors que la partie proximale est plus fine et plus cylindrique.
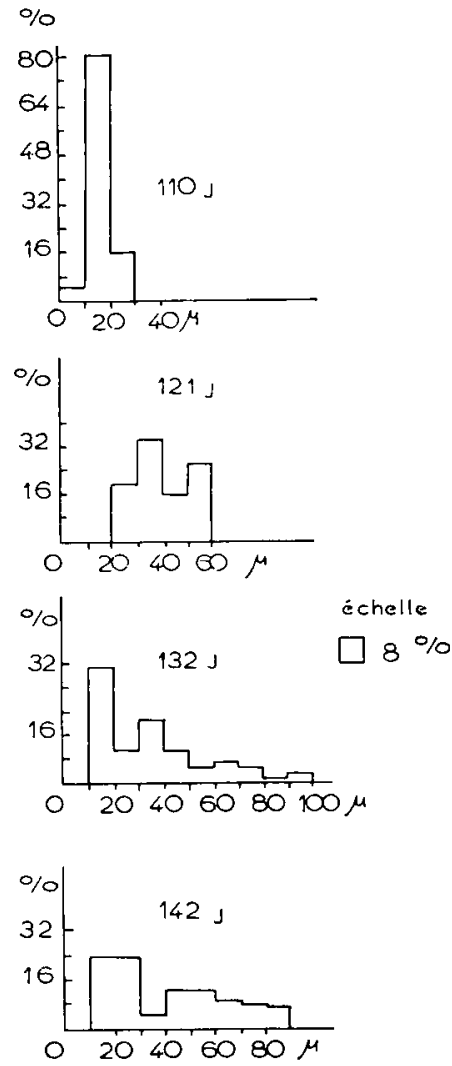

FIG. 22. - Variation avec l'âge du diamètre des fibres chez le fotus Karakul.

\section{TABLEAU VI}

Fréquence du diamètre des fibres

en fonction de l'âge chez l'agneau $n^{\circ} 20$, riche en poils hétérotypiques.

Intervalle de classes en $\%$.

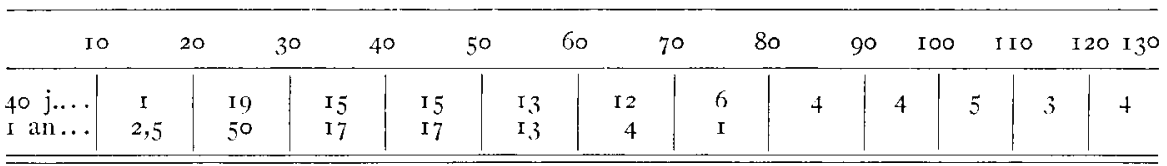

I e tableau VI montre le rétrécissement d'une toison riche en poils hétérotypiques (agneau 20). Les valeurs indiquées représentent le pour- 
centage des fibres ayant leurs diamètres compris entre 10-20, 20-30, ...microns.

3) Cas des individus à poils peu médullés: La toison non médullée est plus homogène, l'histogramme du diamètre présentant detux modes au lieu de trois (fig. I). Mais les processus des modifications avec l'âge sont les mêmes que pour les poils médullés.
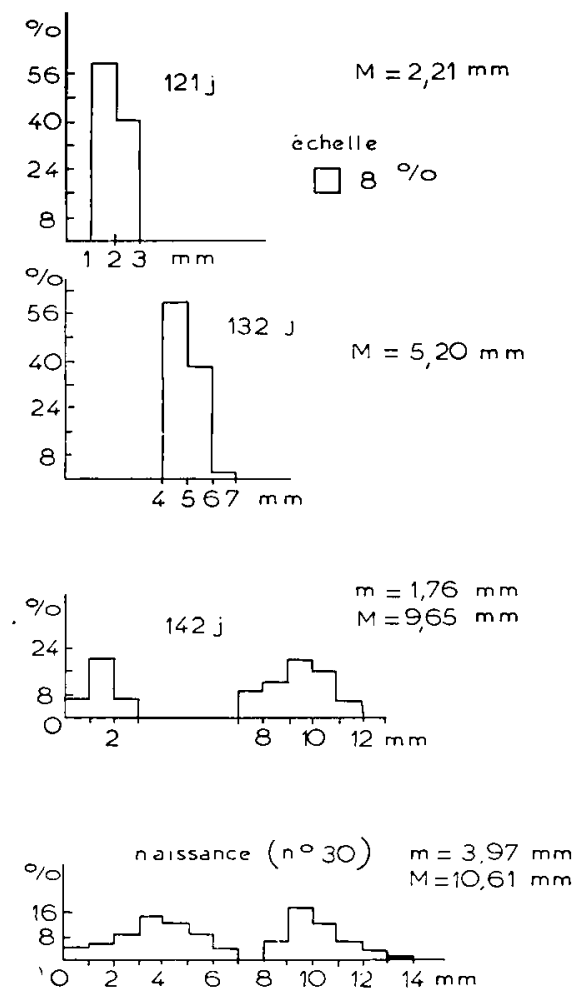

FIG. 23. - Variation de la longueur des fibres avec l'âge chez le foetus Karakul. Remarquer le déplacement de l'histogramme vers la droite ( 2 I I et I 32 jours) et l'apparition du duvet (I 42 jours).

\section{d) Longueur des fibres.}

La figure 23 montre l'augmentation de la longueur des fibres en fonction de 1'âge chez le fotus. Les valeurs extrêmes ainsi que les longueurs moyennes sont indiquées dans le tableau VII.

On remarque sur la figure 23 qu'au $\mathrm{I}_{2 \mathrm{I}} \mathrm{e}$ jour seuls les sommets des poils sont sortis à la surface de la peau d'une longueur moyenne de 2,2I mm. Au $\mathrm{r}_{32} \mathrm{e}$ jour, ces mêmes poils sont déjà sortis d'une longueur moyenne de $5,20 \mathrm{~mm}$, ce qui provoque un déplacement de l'histogramme vers la droite, sans que sa forme change. Au $\mathrm{I}_{4} 2^{\mathrm{e}}$ jour le déplacement vers la droite continue mais l'histogramme est décomposé en deux parties du fait de l'apparition d'une seconde population de fibres (la laine) à 
la surface de la peau. Chez l'agneau à la naissance on retrouve cette seconde population dont la proportion a augmenté au détriment des poils (fig. 6o).

\section{TABLFAU VII}

Variation de la longueur (en $\mathrm{mm}$ ) des fibres primaires et secondaires en fonction de l'âge, chez le foetus Karakul.

\begin{tabular}{|c|c|c|c|c|}
\hline \multirow{2}{*}{$\begin{array}{l}\text { Age foetal } \\
\text { en jours }\end{array}$} & \multicolumn{2}{|c|}{ Fibres primaires } & \multicolumn{2}{|c|}{ Fibres secondaires } \\
\hline & Limites & Moyenne & Limites & Modenne \\
\hline 121 & $1-3$ & $2,2 \mathrm{I}$ & & \\
\hline${ }^{\prime} 32 \ldots \ldots \ldots \ldots$ & $4-7$ & 5,20 & & \\
\hline $\mathrm{I}_{42} 2 \ldots \ldots \ldots \ldots \ldots$ & $7-\mathrm{I}_{3}$ & 9,65 & $0-3$ & 1,76 \\
\hline
\end{tabular}

\section{DISCUSSION}

\section{$I^{\circ}$ Epiderme :}

Chez l'embryon Karakul l'épaisseur de l'épiderme subit une augmentation continue jusqu'à un maximum de $35 \mu$ (6-7 assises cellulaires) au IIoe jour, suivie d'une exfoliation brusque qui ramène l'épaisseur à une douzaine de $\mu$; ce phénomène coïncide avec l'éruption des fibres primaires. Cette constatation est en accord avec celle de Diomidova (I954) qui trouve chez la race Mérinos un maximum de $38 \mu$ (9 couches cellulaires) au moment de l'éruption des fibres et une chute de l'épaisseur de l'épiderme, la réduisant de so fois.

\section{$2^{0}$ Développement folliculaire :}

Nous avons vu que selon Nikoljskr (I932) les premiers germes folliculaires se forment sur la tête de l'embryon Karakul à l'âge de 60 jours. Cette date a été rappelée par HoRNITSCHEK (I938) et par HERRE et WIGGER (I939) qui ne donnent d'ailleurs aucune précision sur la méthode employée. Nous mêmes nous situons cette formation peu avant le $5^{6} \mathrm{e}$ jour (soit environ au $53^{\mathrm{e}}$ jour) de la vie embryonnaire, sur la tête et au $55^{\mathrm{e}}$ jour sur 1'avant-bras (en ne tenant pas compte des germes descils et paupières, etc... qui apparaissent avant le $45^{\mathrm{e}}$ jour). Cette avance de 7 jours sur la date avancée par NikoLJSKI est due au fait que ce dernier a fait ses observations à la loupe binoculaire alors que nous avons procédé à l'observation microscopique après coloration. Au $6 \mathrm{I}^{\mathrm{e}}$ jour, la surface du corps est entièrement recouverte de germes folliculaires avec une densité très faible sur certaines parties du flanc. La croupe se recouvre 8 jours après la tête. Or NIKOLJSKI situe la formation de ces ébauches I5 à $I 7$ jours plus tard.

L'apparition des premiers germes folliculaires chez la race Karakul $\left(53^{\mathrm{e}}\right.$ jour) semble être légèrement en retard par rapport aux races Rom- 
ney ( $49^{\mathrm{e}}$ jour d'après GALPIN, I935) et Kirghiz ( 45 à $5 \mathrm{o}^{\mathrm{e}}$ jour selon TERENTJEVA, I937). Mais ce retard sur la tête est largement compensé par 1a rapidité avec laquelle les germes folliculaires envahissent la surface entière du corps $\left(6 \mathrm{I}^{\mathrm{e}}\right.$ jour chez le Karakul, $64^{\mathrm{e}}$ jour chez le Romney et

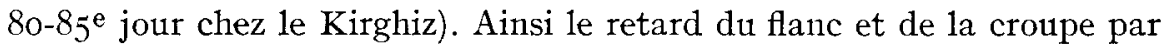
rapport à la tête est de 8 jours pour le Karakul, I 5 jours pour le Romney et de 35 jours pour le Kirghiz.

CARTER (I943) situe au $65^{\mathrm{e}}$ jour de la vie foetale l'apparition des germes folliculaires au niveau du flanc chez le Mérinos, c'est-à-dire 4 jours en retard sur la date que nous avons trouvée pour la race Karakul (tableau VIII). Mais le développement folliculaire chez le fotus Karakul, en avance sur le Mérinos pour la phase pré-triade, se ralentit et le début de la phase triade est atteint en même temps chez les 2 races ( $75^{\mathrm{e}}$ jour). Chez le Karakul, le début de la phase post-triade au niveau du flanc (apparition des follicules secondaires), est en retard de I4 jours sur le Mérinos (roo jour au lieu de 86e). De même il y a un retard d'une semaine pour l'éruption des fibres primaires (I I $3^{\mathrm{e}}$ jour au lieu de I06) et un retard de 9 jours pour la kératinisation des fibres secondaires $\left(\mathrm{I} 24^{\mathrm{e}}\right.$ au lieu de $\mathrm{II} 5^{\mathrm{e}}$ jour).

\section{TABLEAU VIII}

Comparaison entre le développement du système pileux de la race Karakul et celui de la race Mérinos. Ce dernier est emprunté au diagramme publié par CARTER (I943).

\begin{tabular}{|c|c|c|c|c|}
\hline $\begin{array}{l}\text { Étapes du développement folliculaire } \\
\text { au niveau du flanc }\end{array}$ & Karakul & $\begin{array}{l}\text { Mérinos } \\
\text { (Carter) }\end{array}$ & \multicolumn{2}{|c|}{ Phase } \\
\hline Éruption des fibres secondaires & $130 \mathrm{j}$ & - & \multirow{6}{*}{$\begin{array}{l}\text { Phase } \\
\text { post- } \\
\text { triade }\end{array}$} & \multirow{6}{*}{$\begin{array}{l}\text { Néophase } \\
\text { (secon- } \\
\text { dares) }\end{array}$} \\
\hline Kératinisation des fibres secondaires. & I 24 & I 15 & & \\
\hline Éruption de toutes les fibres primaires......... & 15 & 106 & & \\
\hline Eruption des fibres $X$ et $Y \ldots \ldots \ldots \ldots \ldots \ldots$ & I 10 & IO 2,5 & & \\
\hline Kératinisation des fibres primaires .... & 107 & $95-98$ & & \\
\hline Apparition du muscle arrecteur $\ldots$... & & 95 & & \\
\hline Apparition des follicules secondaires .... & 100 & 86 & \multirow{6}{*}{$\begin{array}{l}\text { Phase } \\
\text { triade }\end{array}$} & \multirow{9}{*}{$\begin{array}{l}\text { Protophase } \\
\text { (primaires) }\end{array}$} \\
\hline Glandes sébacées avec toutes les fibres primaires. & 95 & 90 & & \\
\hline Glandes sébacées avec $\mathrm{X}$ et $\mathrm{Y} \ldots \ldots \ldots \ldots \ldots$ & 85 & 82,5 & & \\
\hline Glande sudoripare avec toutes les fibres primaires & 82 & 79 & & \\
\hline Follicules primaires $x \mathrm{X} x$ et $y \mathrm{Y} y \ldots \ldots$. & - & 76 & & \\
\hline Follicules primaires $x \mathrm{X} x$ et $\mathrm{Y} \ldots \ldots \ldots$ & 75 & 74 & & \\
\hline Glande sudoripare avec les follicules $X$ & 70 & - & & \\
\hline Follicules primaires $\mathrm{X}$ et $Y \ldots \ldots \ldots$. & 6,5 & $68-7 \mathrm{I}$ & pré triade & \\
\hline Follicules primaires $X \ldots \ldots \ldots \ldots$ & 630 & 65 & & \\
\hline
\end{tabular}

Par conséquent 1'apparition des germes folliculaires sur la surface du corps chez le Karakul n'est pas en retard sur les autres races, comme l'ont prétendu FröLish et HorNiTSchek (I94I) ; elle est même en avance, si on considère la totalité de la surface du corps. Mais les différentes phases du développement folliculaire sont très lentes chez la race Karakul. 
Nous n'avons pas trouvé de follicules secondaires dérivés tels qu'ils ont été décrits par HARDY et L,YNE (1956).

$3^{\circ}$ Rapport $S / P$.

A partir du roo jour, chez le Karakul, le nombre de follicules secondaires augmente rapidement et la valeur de $\mathrm{S} / \mathrm{P}$ I s'accroît très vite jusqu'aux environs du $I 2 I^{\mathrm{e}}$ jour, et plus lentement par la suite. Cet accroissement rapide s'arrête au IIo jour pour le Romney ; il continue chez le Mérinos jusqu'à la fin de la période embryonnaire (fig. 24). A

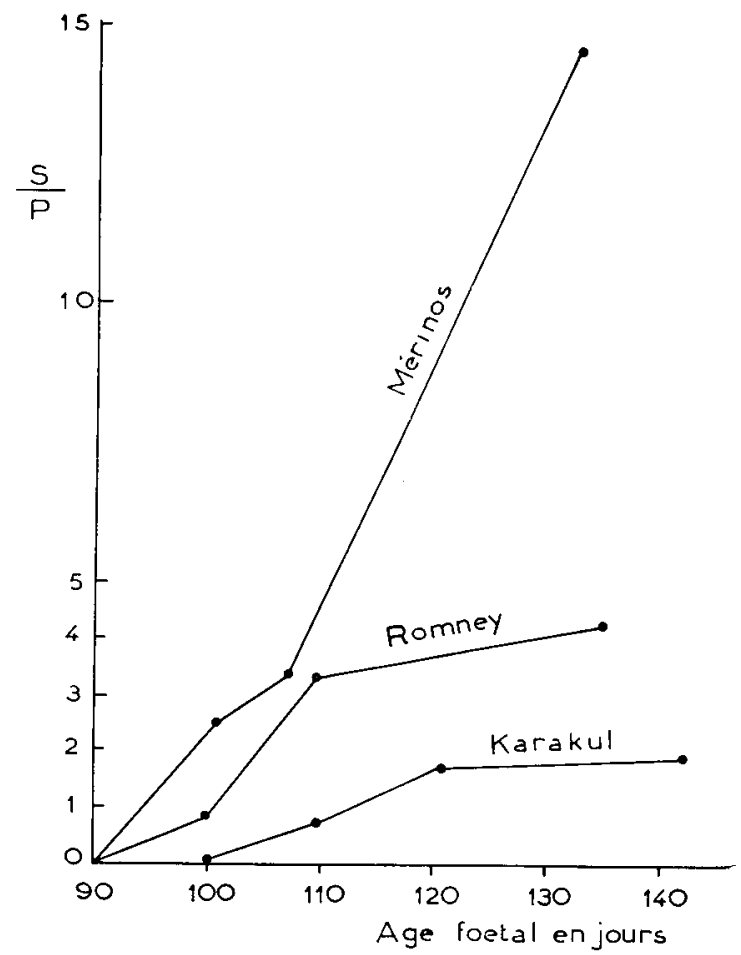

FIG. 24. - Comparaison de la variation avec l'âge du rapport S/P I du foetus Karakul avec celles de la race Romney (Ryder, I956) et de la race Mérinos (Carter et Hardy, 1947, d'après Ryder).

cette période le rapport $\mathrm{S} / \mathrm{P}$ I du Karakul a une valeur 2 fois plus faible que chez le Romney et 9 à Io fois plus faible que chez le Mérinos. Par conséquent le nombre assez faible des fibres secondaires chez l'agneau Karakul ne peut être étranger à la qualité de la boucle.

Ainsi, vu leur faible développement au moment de la naissance, tant par leur nombre que par leur taille, on peut dire que les fibres secondaires ne prennent qu'une part négligeable à la constitution du pelage chez 1'agneau Karakul nouveau-né.

Durant la période post-natale, chez le Karakul, le rapport $\mathrm{S} / \mathrm{P} 2$ subit une augmentation rapide jusqu'au $30^{\mathrm{e}}$ jour et plus faible jus- 
qu'an Ioo $^{\mathrm{e}}$ jour, ce qui est comparable avec certaines autres races ovines :

- Blackface : S/P 2 maximum à 4 mois (Burns, 1953);

- Romney : $\mathrm{S} / \mathrm{P} 2$ maximum à 3 mois (Burns, I949) ;

- Mérinos : S/P 2 maximum à 4 mois et même au-delà (SchinCKEL, I955).

Chez toutes ces races l'augmentation du rapport S/P 2 est rapide durant le $\mathrm{I}^{\mathrm{er}}$ mois et plus lent par la suite. Mais MARGolena (I954) ne trouve pas d'augmentation d $\mathbf{u} \mathrm{S} / \mathrm{P}$ après le $30^{\mathrm{e}}$ jour chez le Karaku1.

$\mathrm{La}$ valeur du rapport $\mathrm{S} / \mathrm{P} 2$ chez l'agneau Karakul diminue légèrement après le $\operatorname{Ioo}^{e}$ jour, ce qui pourrait être dû à la dégénérescence de certains follicules secondaires, comme 1'a supposé BuRns (I949).

$4^{0}$ Densité folliculaire :

Pour la détermination de la densité folliculaire chez le fotus Karakı1 aux différentes étapes du développement foetal, aucune publication n'a été faite à notre connaissance, si ce n'est le travail de TänzER (I928) qui, ne portant que sur trois fotus, ne peut être pris en considération ( $\left.{ }^{1}\right)$.

\section{TABLEAU IX}

Comparaison de la densité folliculaire chez les foetus Karakul et les foetus des races Romney (RYDER, I956) et Mérinos soviétique (DromIDOVA, I954).

\begin{tabular}{|c|c|c|c|}
\hline Age fœtal (en jours) & Karakul (flanc) & Romney (flanc) & Mérinos (dos) \\
\hline $45 \cdots$ & 0 & - & - \\
\hline $5_{62}^{6} \ldots \ldots \cdots \cdots \cdots \cdots \cdots \cdots \cdots \cdots \cdots \cdots$ & o & - & - \\
\hline 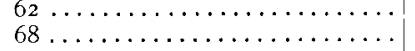 & $\begin{array}{l}\text { I I. } \\
28\end{array}$ & - & - \\
\hline${ }_{75} \ldots \ldots \ldots \ldots \ldots$ & 50 & 24 & 二 \\
\hline 85 & 90 & - & - \\
\hline 90 & - & 62 & I 80 \\
\hline 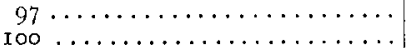 & 75 & - & - \\
\hline $\begin{array}{l}\text { I00 } \ldots \cdots \\
\text { 105 } \ldots . .\end{array}$ & E & IT 9 & 236 \\
\hline 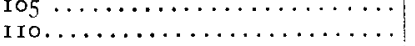 & $\overline{118}$ & $\overline{175}$ & 401 \\
\hline 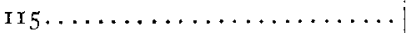 & IIO & - & 463 \\
\hline I $2 \mathrm{I} \ldots \ldots \ldots \ldots \ldots \ldots$ & 97 & - & - \\
\hline I $28 . \ldots \ldots \ldots \ldots \ldots \ldots$ & - & - & 599 \\
\hline 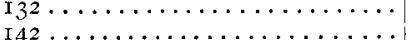 & $\begin{array}{l}90 \\
84\end{array}$ & $\overline{-}$ & $45^{6}$ \\
\hline Naissance............................ & 77 & - & $3 \mathrm{II}$ \\
\hline
\end{tabular}

La densité folliculaire totale chez le fotus Karakul est beaucoup plus faible que chez le fottus Mérinos (tableau IX). Ce tableau montre une augmentation brusque de la densité folliculaire totale après le $100^{\mathrm{e}}$ jour au niveau du flanc, pour le Karakul, entre le $90^{\mathrm{e}}$ et le Ioo $^{\mathrm{e}}$ jour pour le Romney (Ryder, I956) et le Mérinos (Diomidova, I954). Elle est dûe à l'apparition en masse des follicules secondaires.

(1) Fœtus de ı1o jours : densité : 85,5; Fœtus de I I 2 jours, 59 ; - Fotus de I45 jours, 53,05 . 
$5^{\circ}$ Diametre des fibres :

Chez le fœetus et l'agneau Karakul, le diamètre des fibres n'a été mesuré que par TäNzER (I928) qui a construit la courbe de fréquence des diamètres chez le fœetus, l'agneau et l'adulte. Alors que pour l'adulte nous avons trouvé un résultat analogue, chez le fœtus et l'agneau nos résultats sont en contradiction avec ceux de TäNzER. Cet auteur trouve pour le Breitschwanz (d'ailleurs sans précision del'âge) une courbe normale avec un mode situé entre $I 8$ et $20 \mu$, la dispersion des diamètres s'étendant de 6 à $48 \mu$. Or, la courbe de fréquence des diamètres des fibres chez le foetus Karakul n'est normale qu'avant le $I I 5^{\mathrm{e}}$ jour, où seules les fibres $X$ peuvent être mesurées. Mais à partir du II $5^{\mathrm{e}}$ jour, la courbe est bimodale jusqu'au I $3^{\mathrm{e}}$ jour (apparition des fibres $x$ ) pour être trimodale par la suite, du fait de la kératinisation des fibres secondaires. La courbe des diamètres du Breitschwanz ne peut donc être que bi ou trimodale car on ne peut appeler Breitschwanz que les peaux de fœtus Karakul âgés d'au moins I25 jours. Chez l'agneau TÄNZER trouve également une courbe à peu près normale avec un sommet de faible hauteur à $I_{5} \mu$. Or, tous les agneaux que nous avons étudiés montrent une courbe bi ou trimodale avec une prédominance du sommet relatif aux fibres secondaires. Il en résulte que les fibres secondaires (duvet) qui sont pourtant les plus nombreuses manquent dans la prise d'échantillon de TäNzER qui a récolté ses échantillons à l'aide de ciseaux.

60 Longueur des fibres:

Le point le plus délicat des mensurations est la mesure de la longueur des fibres courtes et incurvées du fœtus et de l'agneau Karakul. Nous avons vu que les méthodes employées jusqu'ici n'étaient pas appropriées au matériel. Par conséquent, les résultats trouvés par MEHNER (I92I), Nicov (I929), Iwanov (I932) et PfeIfer (I953) ne peuvent être retenus qu'à titre d'estimation.

\section{CONCLUSION}

L'épiderme de l'embryon Karakul au niveau du flanc s'épaissit par augmentation du nombre de ses assises cellulaires jusqu'au I $^{\mathrm{e}}$ jour, âge qui correspond à l'éruption des poils à ce niveau. L'épaisseur de l'épiderme diminue alors brusquement, passant de 35 à $\mathrm{I} 3 \mu$ par exfoliation des assises cellulaires. Par la suite, son épaisseur reste constante et le nombre d'assises cellulaires limité à 2 . Au contraire, le derme augmente constamment d'épaisseur durant le développement embryonnaire.

Les premiers germes des follicules primaires apparaissent, chez le fœtus Karakul, peu avant le $56^{\mathrm{e}}$ jour sur la tête et peu avant $1 \mathrm{e} 62^{\mathrm{e}}$ jour sur le flanc.

La disposition des follicules primaires, chez le fœtus Karakul, ré- 
pond à la loi générale du groupement par triade avec ses variantes appelées " couple " (X accompagné d'un seul $x$ ) et " solitaire ".

L'éruption des premières fibres issues des follicules primaires a lieu au $95^{\mathrm{e}}$ jour sur la tête. Les dernières fibres primaires émergent sur le flanc et la croupe entre le I $^{\mathrm{e}}$ et le $\mathrm{I} \mathrm{I}^{\mathrm{e}}$ jour de la vie foetale. L'éruption des fibres se fait par une région voisine de l'extrémité de la fibre ; ce n'est que plus tard que l'extrémité arrive à se dégager des cellules épidermiques où elle était restée bloquée.

Les premiers follicules secondaires se forment au $85^{\mathrm{e}}$ jour sur la tête et entre le 97 et le I Io $^{\mathrm{e}}$ jour (probablement au $\mathrm{Ioo}^{\mathrm{e}}$ jour) au niveau du flanc. L'éruption des fibres secondaires à la surface de la peau a lieu entre le $I r^{\mathrm{e}}$ et le $I I 5^{\mathrm{e}}$ jour sur la tête et au $\mathrm{I} 3^{\mathrm{e}}$ jour sur le flanc, mais la tête est souvent dépourvue de follicules secondaires.

Le développement du follicule pileux chez le Karakul se passe dans ses grandes lignes, de la même façon que chez les autres races ovines.

L'ébauche de la formation des follicules pileux sur la tête de l'embryon Karakul est un peu moins précoce que chez les autres races ovines, mais elle est plus précoce que ces dernières pour le reste de la surface du corps.

Le développement folliculaire chez le fœtus Karakul est très actif durant la phase pré-triade, mais il se ralentit pendant la néophase (phase post-triade) et accuse ainsi un retard sur les autres races ovines, notamment le Mérinos.

Les rapports $\mathrm{S} / \mathrm{P}$ I et $\mathrm{S} / \mathrm{P} 2$ augmentent constamment avec 1'âge fœtal. Après la naissance de l'agneau, le rapport $\mathrm{S} / \mathrm{P} 2$ augmente rapidement jusqu'au premier mois ; cette augmentation continue mais avec un rythme plus lent jusqu'au $3^{\mathrm{e}}$ mois.

Les rapports S/P chez le Karakul sont très faibles par rapport aux autres races.

Pendant la période qui s'écoule depuis l'apparition des follicules jusqu'à la naissance de l'agneau, la densité folliculaire augmente d'abord et diminue ensuite lorsque la vitesse d'accroissement de la peau devient supérieure à la vitesse de différenciation folliculaire.

La densité pileuse chez le Karakul est beaucoup plus faible que chez 1e Mérinos, ce qui peut être considéré comme un facteur favorable à l'incurvation et au bouclage réguliers de la toison de l'agneau Karakul.

Le diamètre des fibres augmente avec l'âge depuis leur formation jusqu'au $25^{\mathrm{e}}$ jour de la vie post-natale, puis reste plus ou moins constant pendant deux à trois mois à partir duquel il diminue jusqu'à cinq à six mois pour rester constant par la suite.

Il n'existe pas de limite précise de diamètre différenciant les fibres primaires des fibres secondaires.

La courbe de fréquence des diamètres des fibres présente chez le 
fœtus Karakul, un seul sommet jusqu'au $I I 5^{\mathrm{e}}$ jour, deux sommets entre le $I I 5^{\mathrm{e}}$ et le $13^{\mathrm{e}}$ jour, et en général trois sommets par la suite jusqu'au $3^{\mathrm{e}}$ à $5^{\mathrm{e}}$ mois de la vie post-natale ( 2 sommets dans le cas des poils non médullés). Chez l'adulte, on ne trouve que deux sommets relatifs aux fibres primaires et secondaires.

En ce qui concerne la longueur des fibres chez le foetus Karakul, on distingue sur la courbe de fréquence, une seule population jusqu'au $135^{\mathrm{e}}$ jour et deux populations par la suite. Ces deux populations continuent à exister chez l'agneau et correspondent aux fibres primaires et secondaires. Les publications anciennes n'ont pu mettre en évidence les différents sommets de ces courbes de fréquence du fait qu'il n'existait pas jusqu'ici une méthode précise appropriée au matériel. Grâce à la méthode de projection que nous avons mise au point, nous avons pules mettre en valeur.

Ainsi à la naissance la toison de l'agneau Karakul présente par rapport aux autres races étudiées des caractères originaux dont l'incidence sur la qualité de la fourrure est probable. C'est ainsi que l'apparition tardive des follicules secondaires et la valeur relativement faible $\mathrm{du}$ rapport $\mathrm{S} / \mathrm{P}$ fait que la boucle est pratiquement constituée uniquement de fibres primaires incurvées mais non frisées comme nous le verrons au chapitre suivant.

\section{CHAPITRE III}

\section{LA BOUCLE DE L'AGNEAU KARAKUL}

\section{HISTORIQUE}

I'agneau Karakul est caractérisé par sa fourrure brillante comportant des boucles régulières constituées par des fibres plus ou moins incurvées. L'étude des causes de la formation de ces boucles a fait l'objet d'un certain nombre de travaux.

De nombreux auteurs ont invoqué la forme de la section des fibres. Certains (l), tels que FriTSCH (I92I) et LosSEN (I92I) lui attribuent un rôle important alors que d'autres comme FREDERIC (Igo6) la considèrent comme un facteur parmi beaucoup d'autres.

L'absence de la moelle est considérée par TäNZER (I928) comme un facteur favorable à la formation de la boucle.

Mais c'est surtout la forme du follicule pileux qui a retenu l'attention des auteurs :

Ainsi Tänzer (I928) affirme que l'incurvation du follicule est la cause de la formation de la boucle. Le follicule au début de sa formation

(1) D'après Pfeifer, ig29. 
est rectiligne et orienté obliquement; peu à peu il s'incurve jusqu'à atteindre la forme d'un sabre, ce qui prépare la future incurvation du poil. TäNZER explique la formation du coude de la base du follicule comme le résultat de l'arrêt de la poussée du follicule vers la profondeur par la présence du tissu adipeux et l'augmentation de la tension de la peau qui oppose une résistance au développement du follicule. Celui-ci après avoir acquis la forme du sabre se redresse à nouveau grâce au développement et à l'augmentation de l'épaisseur des différentes couches de la peau.

Ce redressement a pour effet le déroulement de la boucle.

PFFIFER (I929) confirmant les opinions de TÄNZER trouve, en outre, un deuxième coude dans le tiers supérieur du follicule ayant un effet inverse et qui serait la cause du débouclement. Il fait passer un fil métallique à travers un tube de verre présentant des coudes et obtient à la sortie, le fil enroulé.

Cependant si on ne retrouve pas sur les autres races le type de boucle propre au Karakul, les fibres de tous les agneaux n'en sont pas moins bouclées ou frisées et de nombreux auteurs ont cherché à en déterminer les causes.

L'idée la plus ancienne sur la frisure de la laine est celle de PerRAULT DE JOTEMPS (rapporté par DUCLERT, I888) qui en I824, dans un traité sur la laine de mouton écrivait : " la forme dı brin est modifiée par la configuration du pore de la peau qui sert de moule. Ainsi, le poil sera fin, lisse, ondulé, etc... suivant que le pore sera étroit, droit ou tortueux ".

Nathusius (I866) confirme que chez le mouton le poil droit est issu d'un follicule droit et que le poil frisé provient d'un follicule contourné. I1 invoque également l'action de l'humidité (sécrétion des glandes sébacées et sudoripares), et de la chaleur (température du corps) sur la frisure de la laine. Mais il nie l'influence de la forme de la section du poil et de la présence de la moelle.

STICKERT (I887) ne retrouve pas les follicules en spirale décrits par Nathusius, mais il déclare que les forces agissant à l'intérieur du follicule, du fait de l'existence d'un coude au-dessus du bulbe, ont une résultante qui est celle d'une spirale.

DUCLERT ( 1888 ) pense qu'il y a une relation manifeste entre l'ondulation du brin de laine et l'enroulement en spirale de son follicule. I1 considère le coude observé par STICKERT comme une conséquence de 1'enroulement en spirale du follicule.

VIGIER (1904) à la suite de ses recherches comparatives sur le follicule pileux de nègres et de moutons met en évidence l'influence de la "crête semi-circulaire " existant entre le bulbe et le renflement de la gaine épithéliale externe et s'exprime ainsi : le follicule tout entier ne sert pas de matrice au poil pour lui imposer sa forme, mais c'est dans la "crête 
semi-circulaire oblique " (épaississement de la gaine fibreuse) qu'il faut voir la cause première de la frisure du poil ; l'enroulement du follicule lui-même est un phénomène secondaire.

WildMan (I932) indique chez les races ovines britanniques que la forme frisée et la position excentrique de la fibre dans le follicule peuvent être déterminées par la localisation du " growing-point " ( ${ }^{1}$ ) de la fibre et de la gaine épithéliale interne du côté convexe de la déflexion du bulbe. Cette localisation peut changer et par suite causer la réversion de la croissance spirale de la fibre et celle de la déflexion du bulbe.

Auber (I95I) rappelle les notions suivantes:

- les fibres courbes sont produites seulement par des follicules incurvés avec bulbe déjeté;

- la déflexion du bulbe coïncide avec le décentrement et une différenciation segmentaire et non périphéro-axiale du cortex;

- les follicules droits ou courbes avec bulbe non déjeté produisent des fibres droites avec différenciation périphéro-axiale typique à symétrie axiale.

Ein accord avec Wilphan il admet qu'un déplacement du " growingpoint " influe sur la courbure de la fibre.

Selon Auber la courbure du follicule au-dessus du coude peut ne pas être la cause mais l'effet de la courbure de la fibre.

D'autres auteurs invoquent des caractères propres de la fibre pour expliquer sa frisure :

BRown (I853) (selon DucleRT, I888) attribue la frisure de la laine à la section ovale qu'il a observée chez la toison des moutons.

DUERDEN (I927) pense que l'ondulation des brins de laine des toisons très denses est due en partie à l'aplatissement de la spirale primaire et à l'inégale croissance latérale de la fibre.

Selon Astbury (I942) au cours du phénomène de la kératinisation, chaque réajustement chimique contracte progressivement la structure fibrillaire. Ainsi la fibre fléchirait du côté où la kératinisation a d'abord commencé.

Les recherches récentes de Horrio et Kondo (I953) montrent qu'une fibre de laine ondulée teinte par un colorant basique prend la teinture presque uniquement du côté extérieur à la courbure. Le côté intérieur (concave) se colore de préférence par les colorants acides. D'où l'idée que la structure hémicylindrique de la fibre est en rapport avec l'ondulation de celle-ci.

Mercer (I953) a appelé orthocortex la partie accessible aux colorants basiques et paracortex la partie restante. Il démontre en outre (1954) par des autoradiographies que l'orthocortex est moins riche en cystine que le paracortex.

(1) Par "growing-point" on entend une localisation maximale des divisions cellulaires. 
Mais les auteurs ont fréquemment confondu deux phénomènes bien distincts : l'incurvation du poil et le bouclage qui est un phénomène d'accompagnement d'un certain nombre de poils.

Tandis que tous les auteurs anciens ont invoqué les causes de l'incurvation du poil pour expliquer la formation de la boucle, l'importance de l'orientation des follicules dans l'organisation de la boucle n'a été qu'à peine entrevue par HoRNITSCHEK (I938).

Cet auteur trouve chez l'agneau Karakul, que certaines dispositions folliculaires des poils sont caractéristiques de certains types de boucle. Il y aurait donc un rapport entre l'organisation des follicules et le type de la boucle.

HaEcker (I9I8) et I,Uwig (I92I) (') ont tenté d'expliquer 1'orientation des groupes de follicules par le mode de croissance de la peau. Ludwig suppose que chaque petit élément de la peau croît avec une rapidité inégale dans les différentes directions : ceci donnerait naissance à des ondes de croissance rapide et des ondes de croissance lente qui provoquent l'orientation des groupes de follicules.

HoRNitscheK (I938) confirme ces inégalités dans la croissance dans la peau par la différence de densité pileuse qu'il constate sur des portions voisines de peau.

HERRE et WigGER (I939) défendent l'existence de secteurs à croissance différente dans la peau du fœtus Karakul. Ils affirment qu'avec 1'âge les différences locales de tension disparaissent progressivement, ce qui entraîne la disparition de la déflexion et de la courbure des follicules. Chez le porc Mangalica et les Caniches les tensions locales persistent ce qui explique le bouclage permanent de leurs poils.

Une autre explication du changement d'orientation des follicules est donnée par les plis de la peau. Cette opinion est défendue par SchivaLd, Nicov (I929) et HorNiTschek (1). Nicov va plus loin en affirmant que les plis de la peau formeraient les limites des boucles.

Enfin, certains auteurs ont trouvé qu'il y avait une relation entre la qualité de la boucle et les caractéristiques des fibres qui la constituent.

Ainsi Nicov (I930) trouve une relation entre le pourcentage de duvet et la qualité de la boucle, le pourcentage de duvet étant plus fort dans les mauvaises boucles.

JAHN (I923) indique une relation positive entre le diamètre du poil et la qualité de la boucle, les gros poils donnant une bonne boucle. TäNZER (I928) n'est pas de cet avis et selon lui on ne peut pas estimer la valeur de la fourrure par l'étude du diamètre de ses fibres.

Pour la moelle, TÄNzER (Ig28) affirme l'incompatibilité entre la présence de la mœlle et la bonne qualité de la boucle. Nicov (I934) trouve que chez la race 'Tzourcana dont la toison d'agneau est également

(1) D'après HoRnitscheck, 19.38. 
bouclée, le diamètre trop grand ou trop petit de la moelle n'est pas favorable à la formation de la boucle; 1 'indice médullaire optimum est de $\mathrm{I} / 3$.

La forme de la section du poil, selon LOSSEN (I92I) serait en relation avec la qualité de la boucle, ainsi une section en triangle isocèle donnerait une bonne boucle alors que la section ovale formerait une mauvaise boucle.

Nicov (I930) trouve que 1a longueur des poils est plus petite pour les bonnes boucles et plus grande pour les boucles médiocres.

Ajoutons, pour être complet, que LANGLET (I936), JUDIN (I937), Hardy et Simmons (1943) ont montré qu'il y avait une relation très marquée entre l'épaisseur de la peau et la qualité de la boucle, les peaux minces portant les boucles les plus belles.

\section{RESULTATS DE RECHERCHES}

Première PARTIE

\section{BOUCLAGE ET INCURVATION DES POILS}

La boucle de la toison de l'agneau Karakul est une formation instable. Ein effet, cette toison se déboucle peu de temps après la naissance. Les causes qui permettent la formation de la boucle et sa disparition doivent donc se situer pendant la fin de la vie fœetale et les tous premiers jours qui suivent la naissance. Pour essayer d'analyser les facteurs mis en jeu, nous nous sommes livrés à une étude complète de la peau et de la fourrure du $45^{\mathrm{e}}$ jour de la vie foetale jusqu'à 1'âge adulte. Nous avons ensuite repris et confronté, avec l'expérience, les hypothèses qui ont été avancées pour expliquer l'incurvation et le bouclage. Il convient en effet de distinguer la forme d'une fibre considérée isolément et celle des paquets de fibres. Nous réservons le terme d'incurvation quand il sera question d'une fibre et le terme bouclage pour le phénomène d'accompagnement des fibres incurvées constituant la boucle.

\section{A. - Bouclage des poils, orientation des follicules et mécanisme de la formation du dessin de la fourrure.}

On sait que les boucles de l'agneau diffèrent les unes des autres par la taille, par la forme et par l'orientation.

Ainsi remarque-t-on sur la peau, des dessins différents suivant la disposition des boucles les unes par rapport aux autres.

On peut se demander d'où vient cette disposition particulière. 


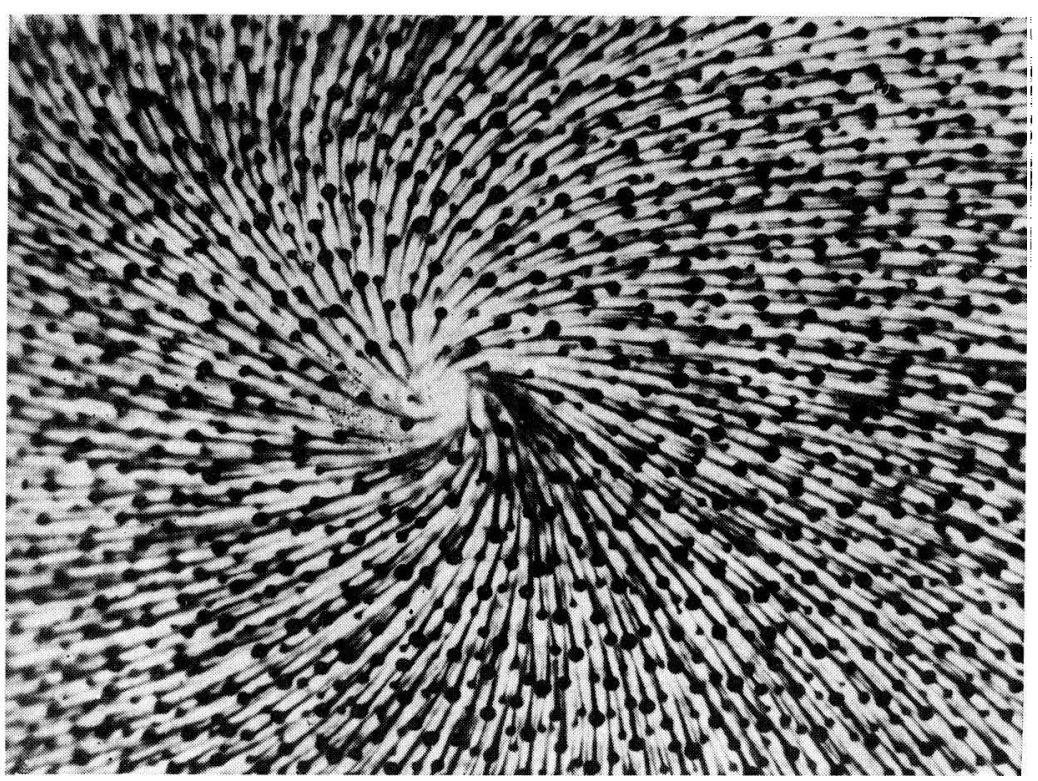

Fig. 25. - Fotus Karakul de I ro jours.

Point de convergence de l'orientation folliculaire sur le flanc.

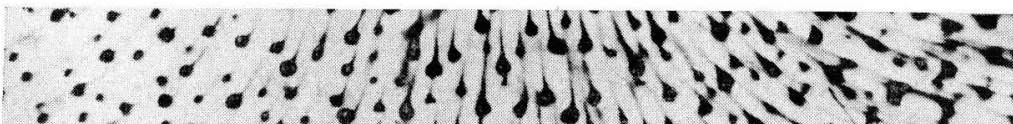
$\therefore$ a

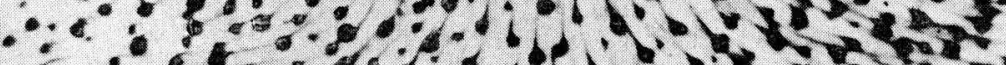

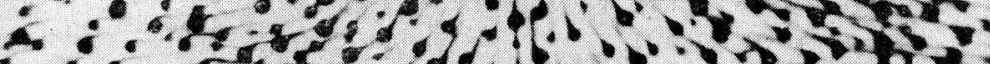

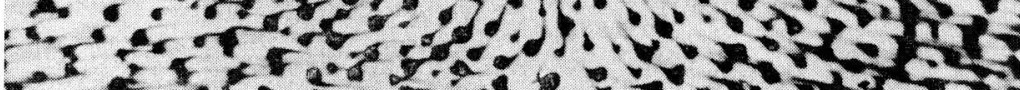

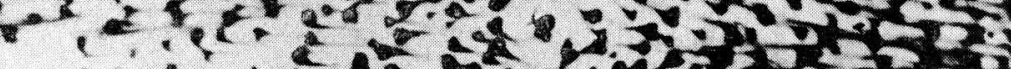

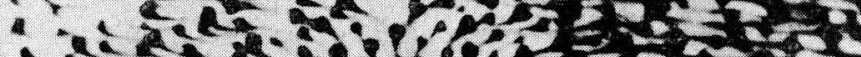

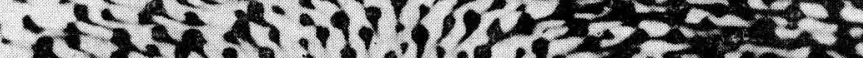

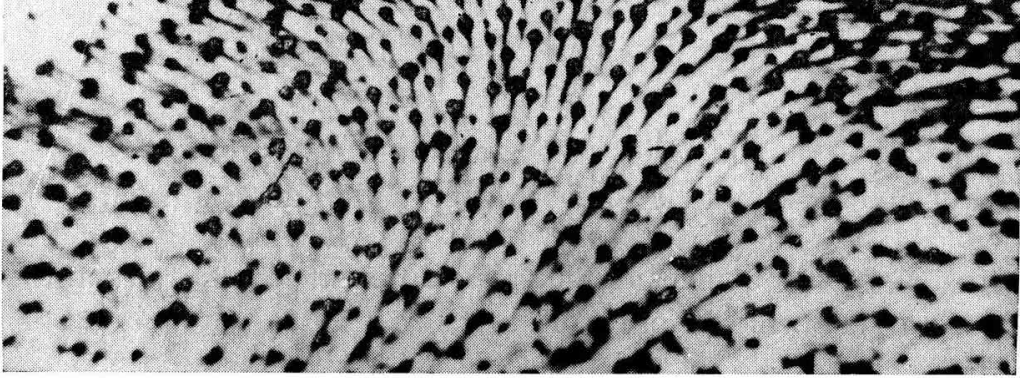

FIG, 26. - Fotus Karakul de IIo jours

Point de divergence et du changement de l'orientation folliculaire au niveau de l'aisselle. 


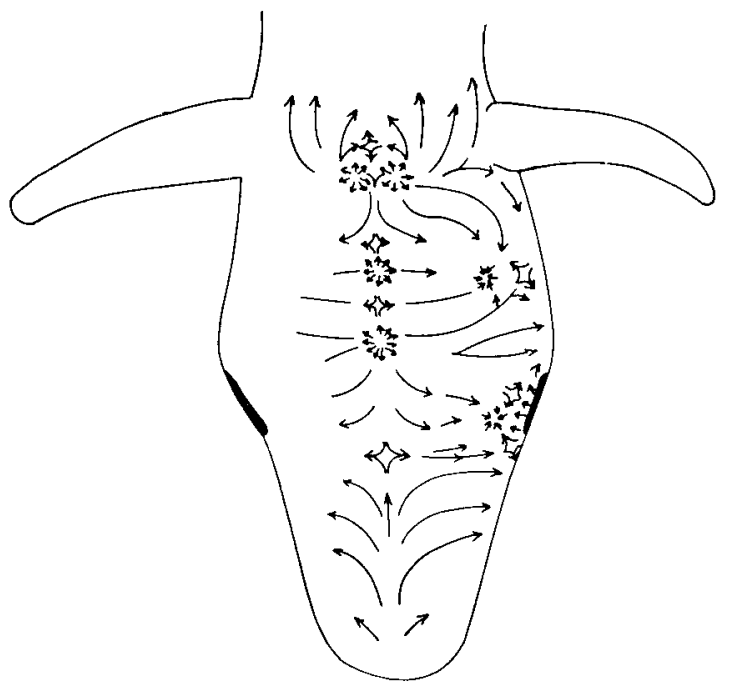

(a)

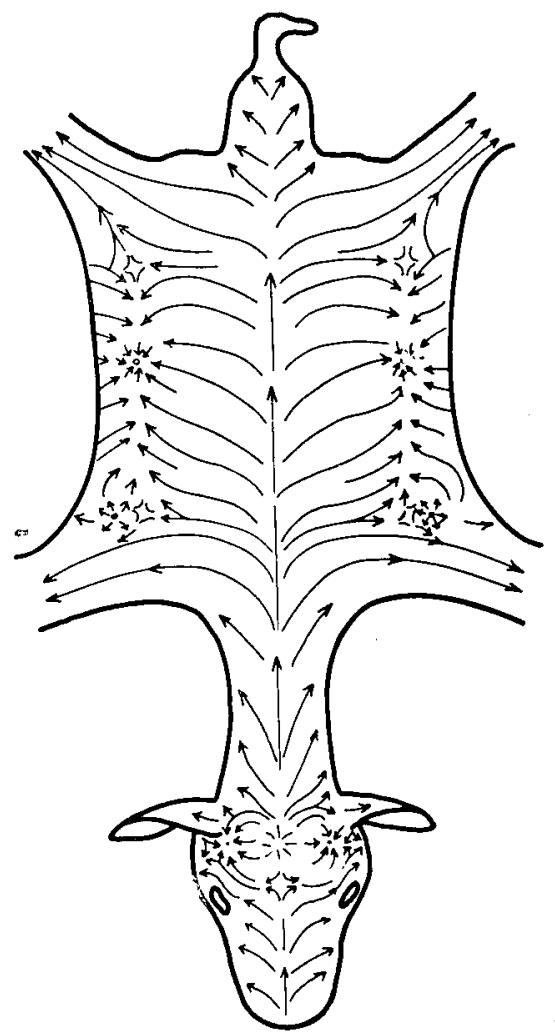

(b)

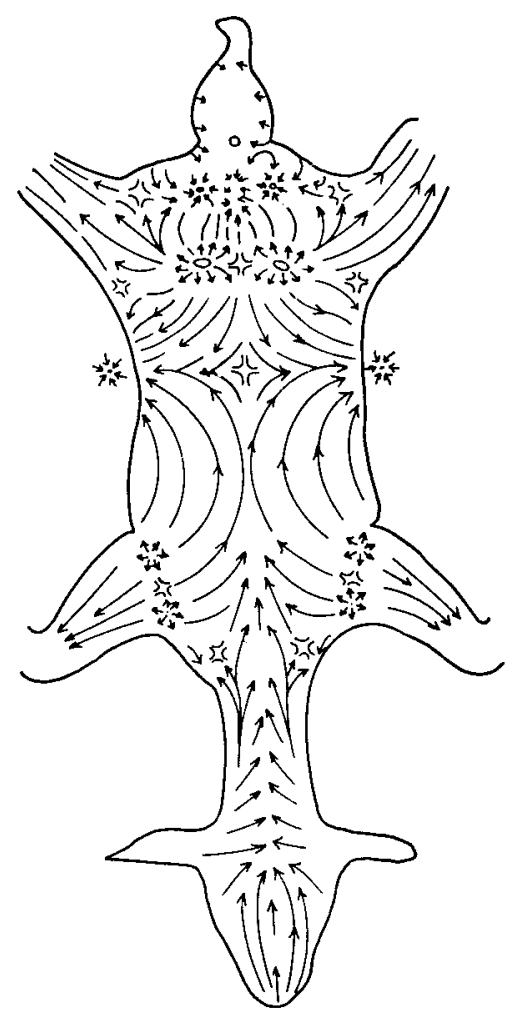

(c)

FIG. 27. - Schéma de l'orientation folliculaire chez le foetus Karakul de i so jours : a) Tête (les deux côtés sont symétriques). - b) Face dorsale. - c) Face ventrale. 


\title{
Io Ébauche de la boucle chez l'embryon.
}

\author{
a) EMBRYON KARAKUL.
}

Pour essayer de résoudre ce problème nous avons utilisé des lambeaux de peau de dimensions relativement grandes (de l'ordre de 20 à $50 \mathrm{~cm}^{2}$ ).
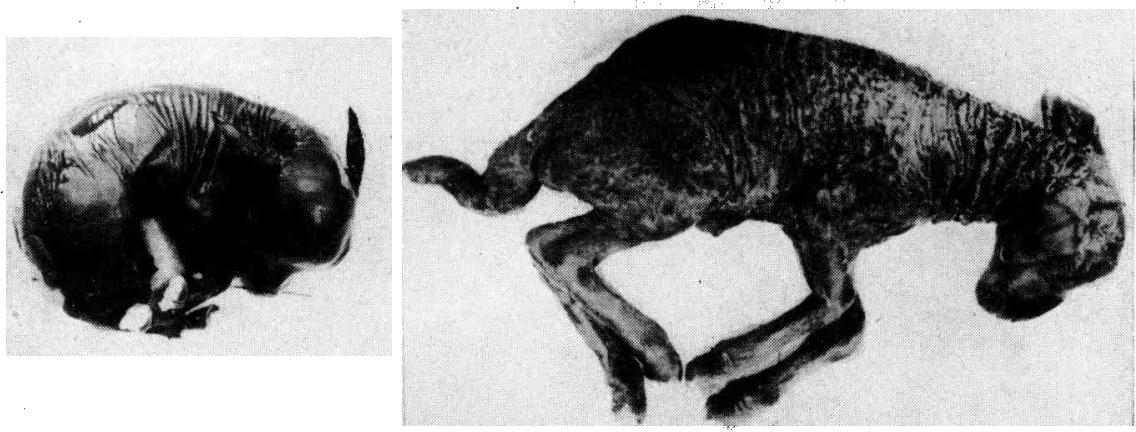

FIG. 28. - Fœtus Karakul de 85 jours. La peau est dépourvue de poils.

FIG. 3o. - Fœtus Karakul de I 32 jours. La fourrure est ondulée. (Breitschwanztéker).

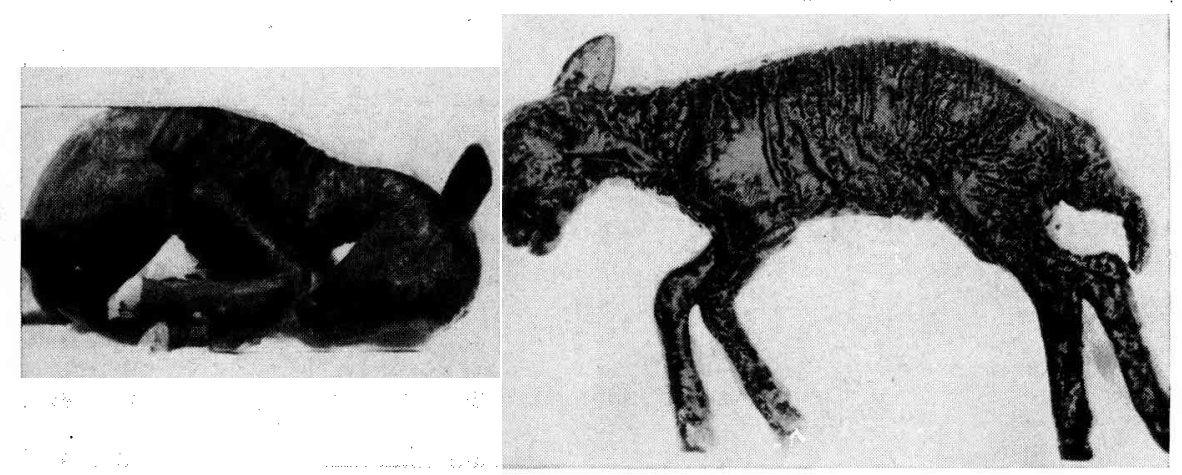

FIG. 29. - Fœtus Karakul de izi jours.

La fourrure est encore lisse (Galyac ou lessac).

FIG. 3I - Fotus Karahul de 142 jours. La fourrure porte déjà des formations bouclées (Teker plus avancé)

Après l'examen à l'oil et à la loupe, ces lambeaux ont été rasés puis éclaircis par le salicylate de méthyle.

L'examen à la loupe montre que les follicules sont initialement dirigés de la tête vers la queue sur les lignes dorsale et ventrale. De part et d'autre de ces lignes et symétriquement les follicules ont tendance à prendre une direction perpendiculaire à ces axes. Ceci n'est visible en des points 


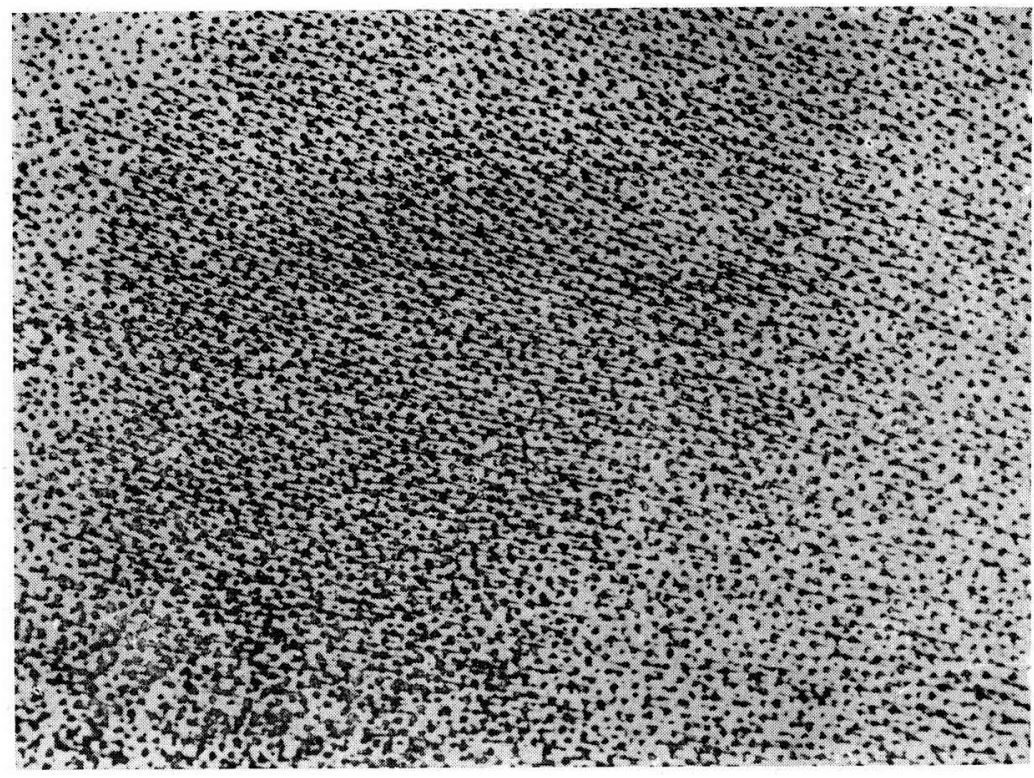

FIG. 32. - Foctus Karakul de II5 jours. Les follicules ont une orientation parallèle sur une zone limitée de la peau.

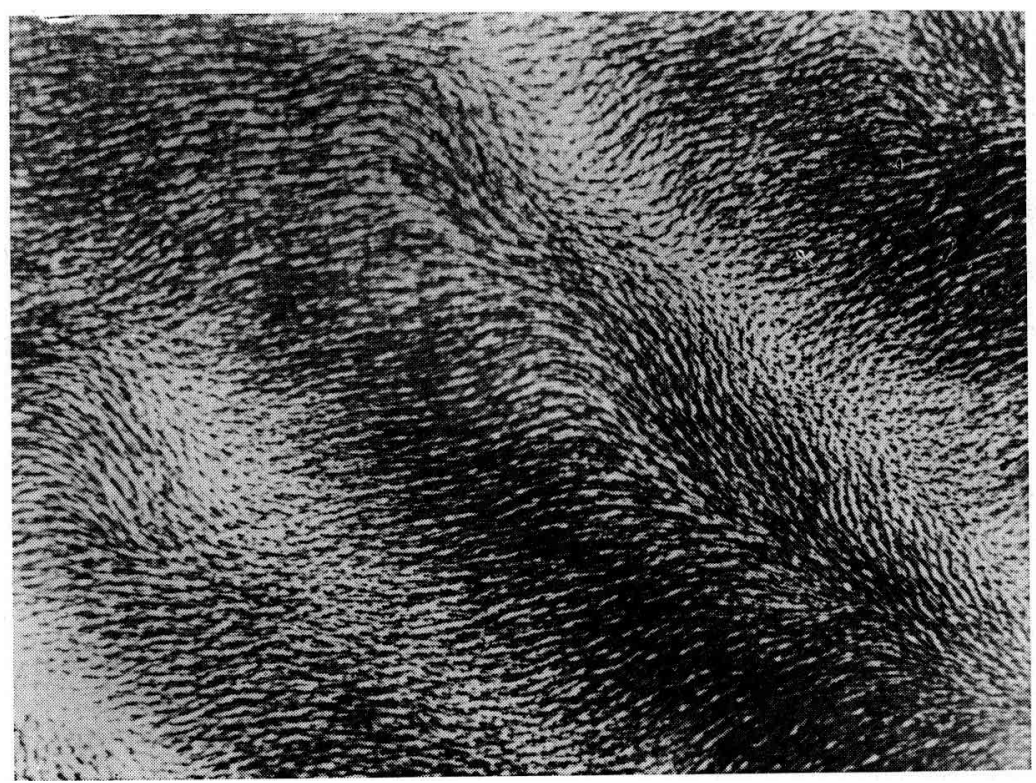

FIG. 33. - Fotus Karakul de I 32 jours. Changement d'orientation des follicules pileux correspondant à l'ébauche des formations bouclées. 
déterminés. Ce sont des points de divergence ou de convergence (fig. 25) ou encore des points de changement de direction des follicules (fig. 26). Leur nombre et leur complication augmente avec l'âge jusqu'au $\operatorname{II} 5^{\mathrm{e}}$ jour de la vie fœtale (fig. 27). Il en existe une quinzaine sur la tête et au-dessus des yeux, 4 à l'aisselle de chaque membre antérieur, deux à

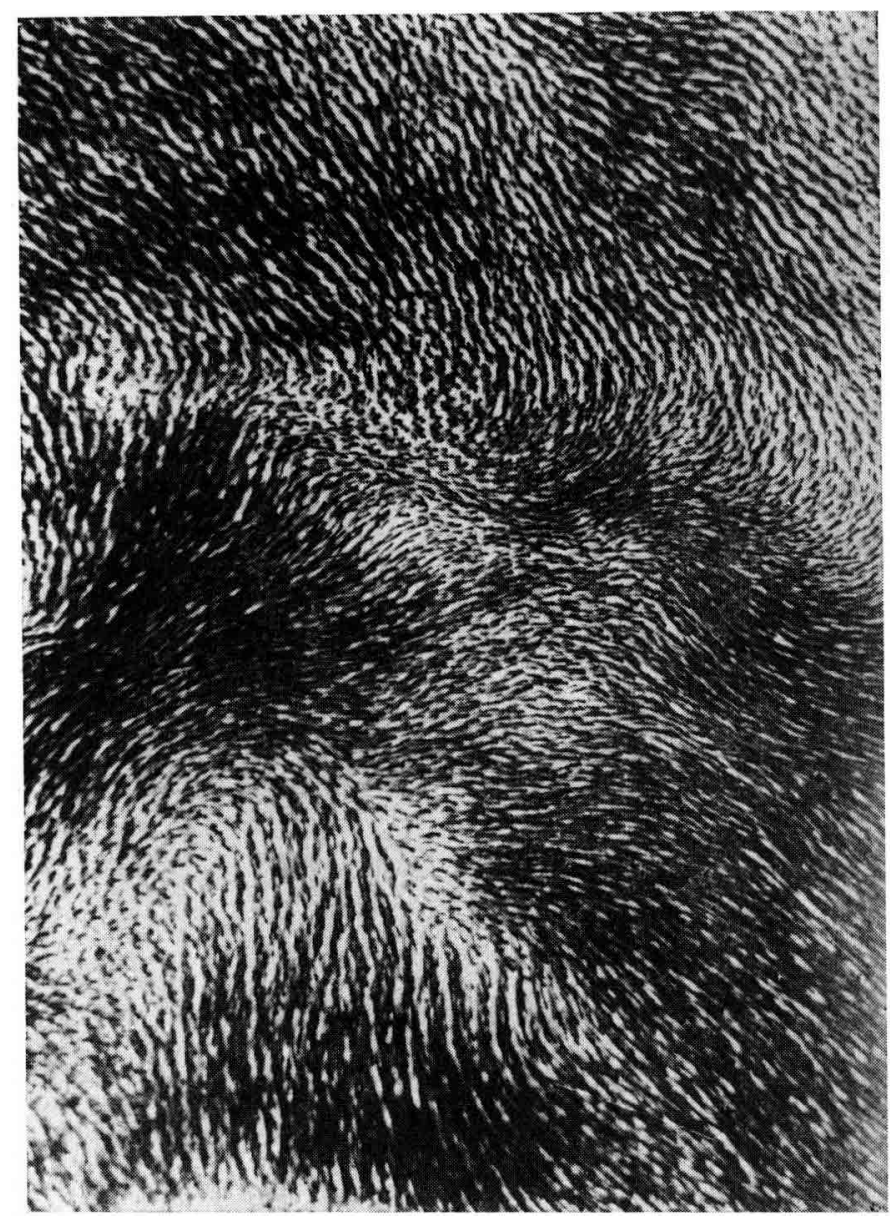

Fig. 34. - Fotus Karakul de I72 jours. Formation de secteurs folliculaires.

l'aisselle de chaque membre postérieur, tune demi-douzaine au niveau des organes sexuels et un au milieu de chaque flanc. Cette disposition des follicules parait être la même pour toutes les races ovines aussi bien chez le fotus que chez l'adulte, selon nos propres observations.

Jusqu'au $\mathrm{II}^{\mathrm{e}}$ jour de la vie fœtale, les follicules d'une zone déterminée de la peau (en dehors des points mentionnés plus haut) sont orientés dans une même direction (fig. 32 et 39). C'est 1'âge auquel les poils sont 
sortis de un à deux mm à la surface de la peau. Par conséquent il ne peut $y$ avoir encore de boucle.

$\mathrm{Au} \mathrm{I}_{20} \mathrm{O}^{\mathrm{e}}$ jour, on observe de légères perturbations dans la direction commune des follicules. De place en place, le parallélisme des follicules est perturbé. Mais, à l'extérieur de la peau, la direction unique des poils n'est pas encore affectée par les perturbations de l'orientation des follicules. La fourrure est donc parfaitement lisse bien que les poils soient incurvés (fig. 29 et 39).

$\mathrm{Au} \mathrm{I}_{3} \mathrm{O}^{\mathrm{B}}$ jour, époque à laquelle un début d'ondulation de la fourrure est visible, on assiste à une accentuation des changements d'orientation des follicules. Au début, ces changements portent sur des zones assez larges plutôt en forme de bandes plus ou moins perpendiculaires à la ligne dorsale (fig. 33 et 39 ). C'est ce qui provoque l'aspect ondulé de la fourrure (fig. 30). Petit à petit ces bandes se trouvent disloquées par de nouveaux changements d'orientation de groupes de follicules.

Ces changements d'orientation ne se font pas d'une façon anarchique et désordonnée. Ils ne se portent pas sur des follicules individuels, mais sur des groupes formant ainsi des secteurs à orientations différentes. A l'intérieur de chaque secteur les follicules conservent une orientation unique (fig. 34 et 39). Ce stade n'est atteint qu'aux environs du $140^{\mathrm{e}}$ jour de la vie fœtale et c'est à cet âge que les poils peuvent former de vraies boucles à la surface de la peau (fig. 3I). Dans certains cas la direction des follicules est complètement inversée. Autrement dit si on considère la projection des follicules sur un plan parallèle à la surface de la peau, l'angle de déviation de la direction des follicules varie de o à $180^{\circ}$.

Les changements d'orientation des follicules affectent leur inclinaison par rapport à la surface de la peau. Cette inclinaison, sensiblement uniforme avant l'apparition de la boucle, devient très inégale à l'intérieur d'une même boucle : elle peut varier de 40 à $80^{\circ}$ (fig. 35 et 36 ).

L'angle d'inclinaison du follicule par rapport à la surface de la peau n'est pas égal à celui de la fibre (fig. 36).

\section{b) Comparaison avec d'autres races}

Parallèlement à ces observations, des embryons de la race Bizeł ont été examinés dans les mêmes conditions d'âge et de technique. Cette race a été choisie à cause de sa toison noire dont les follicules sont visibles, sans aucune coloration, dans le salicylate de méthyle. Jusqu'au I ${ }^{\circ}{ }^{e}$ jour, le fotus Bizet présente comme chez le Karakul, une orientation unique de follicules sur une zone déterminée de la peau (fig. 37).

Au $122^{\mathrm{e}}$ jour, il règne chez le fotus Bizet une véritable anarchie dans 1'orientation folliculaire (fig. 38). Ce désordre a été également constaté chez les fotus de $\mathrm{I}_{32}$ et I 42 jours. Il en est de même pour la race Ile-de-France. La figure 39 montre le schéma de l'orientation folliculaire 


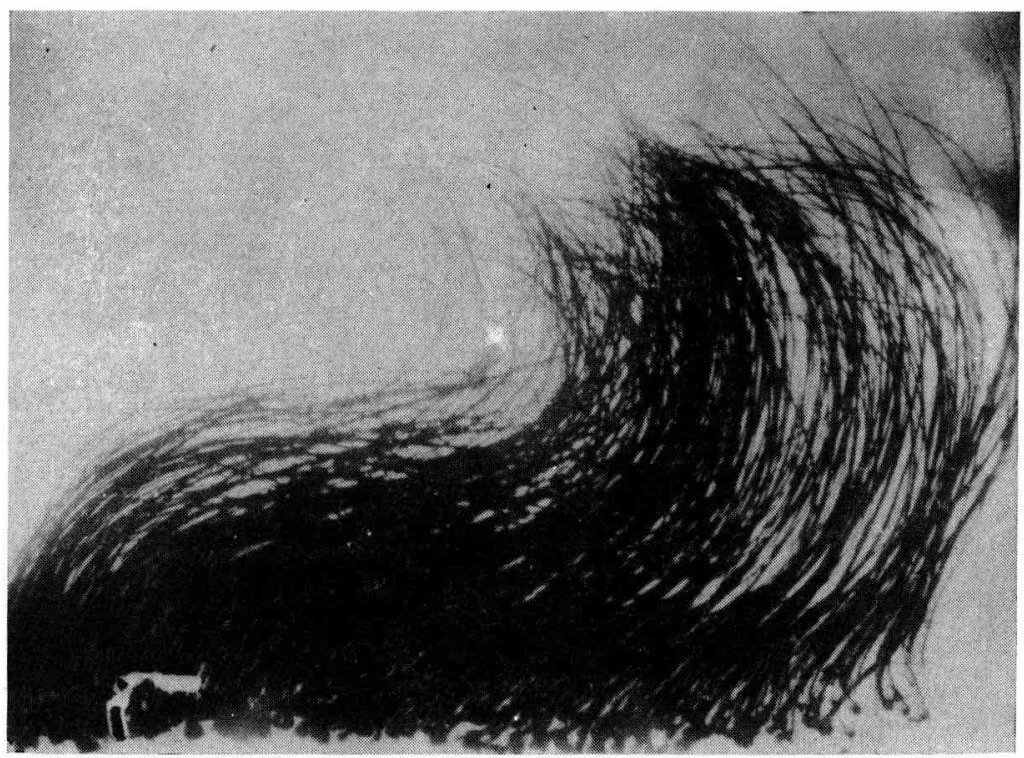

Fis. 35. - Fotus Karakul de I.32 jours (flanc). Début de la formation de la boucle.

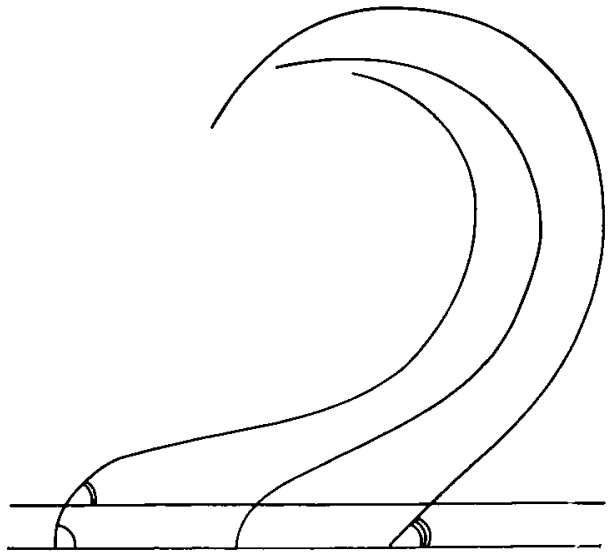

FIG. 36. - Schéma d'une boucle chez le foxtus de I 32 à $I_{42}$ jours. Remarquer l'inclinaison différente des follicules at l'angle que forme la surface de la peau avec le follicule et avec la fibre.

aux différentes étapes du développement embryonnaire chez les races Karakul et Bizet.

\section{$2^{o}$ État de la boucle chez l'agneau nouveau-né.}

\section{a) Agneau Karakul.}

Dans la peau de l'agneau, à la naissance, on observe les mêmes dispositions folliculaires que chez le fotus de I40 jours (fig. 40). Car le maximum de modification dans l'orientation folliculaire a lieu au $145^{\mathrm{e}}$ 


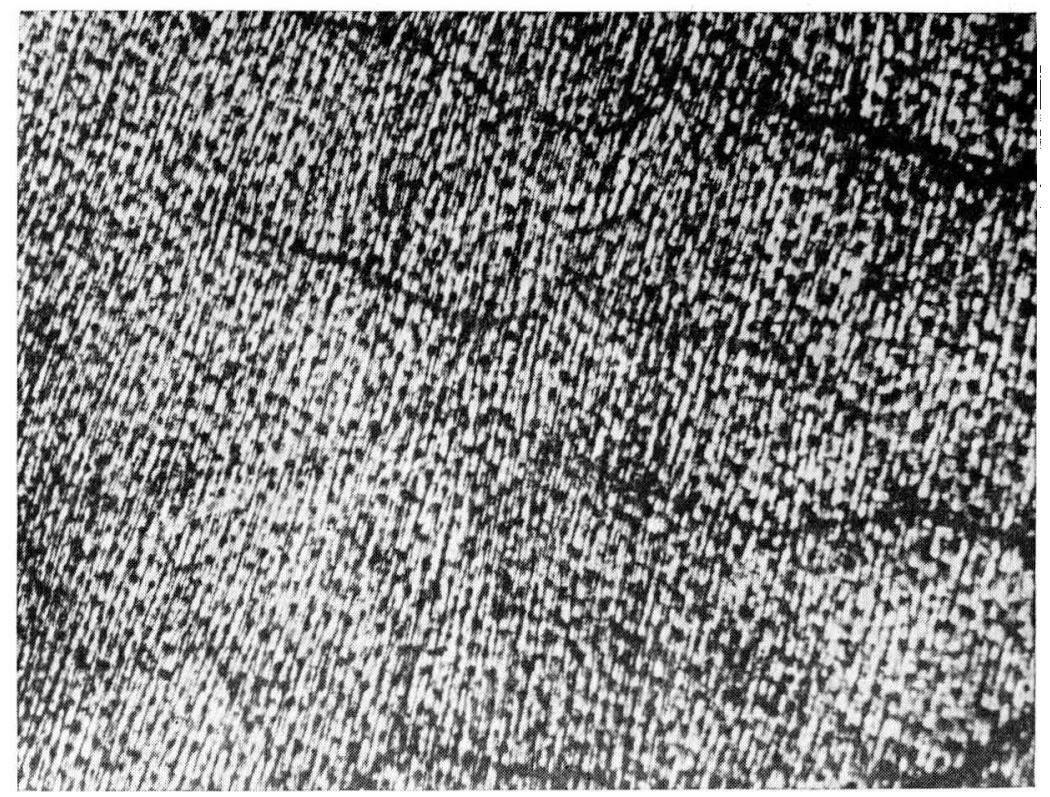

Fig. 3\%. - Footus Bizet de ro jururs. Les follicules ont une orientution parallele sur une zonc limitie de la peau.

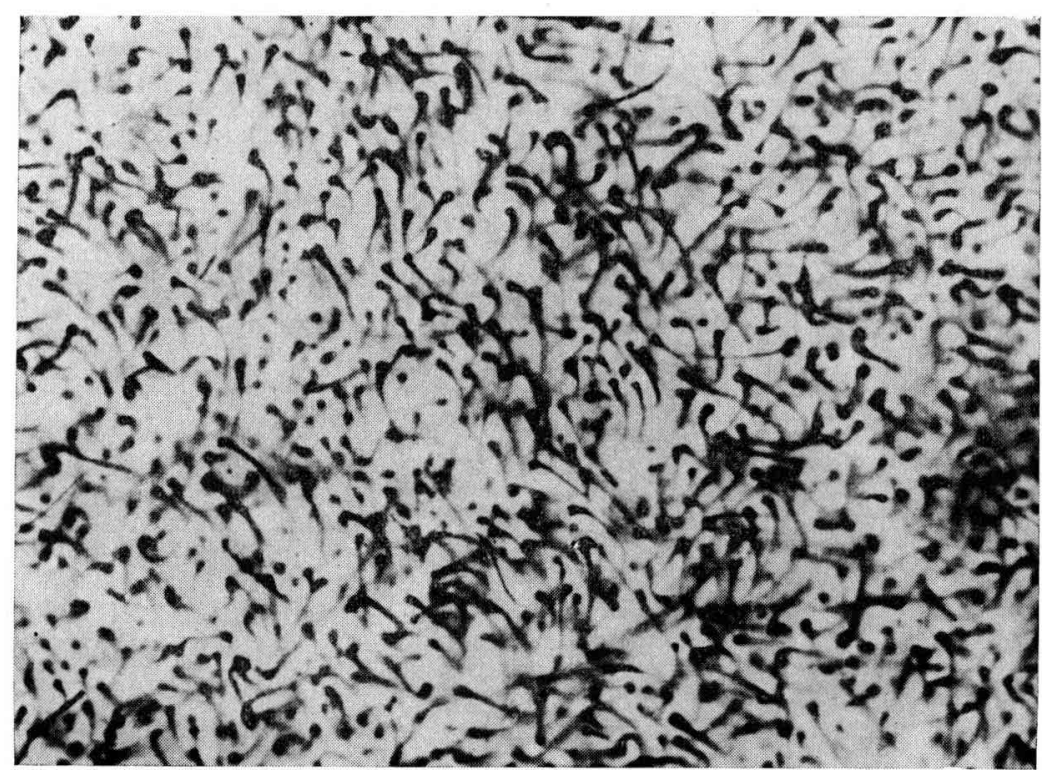

Fig. 38 . - Fotus Bizet de I 22 jours montrant la diversité et l'irrégularité de lorientation des follicules. 


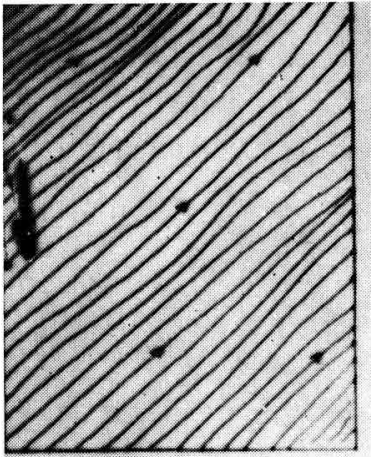

EK $\leqslant$ ns
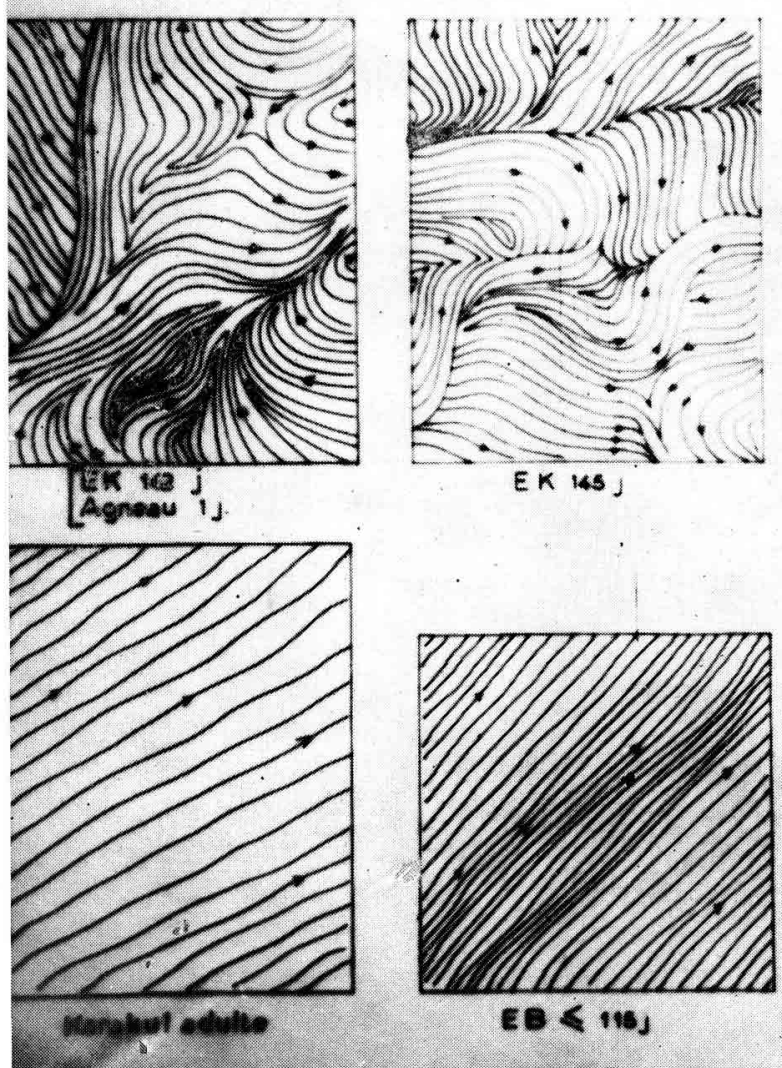

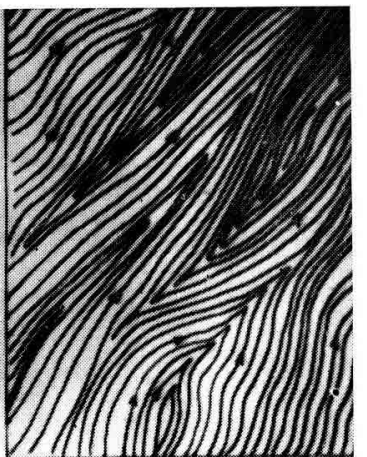

EK 132 s.

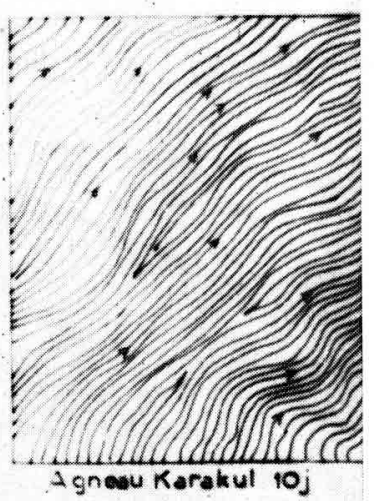

FIG. 39. - Schémas de l'orientation folliculaire aux différentes étapes du développement embryonnaire dans les races Karakul et Bizet.

EK : Embryon Karakul; LB : Embryon Bizet. 


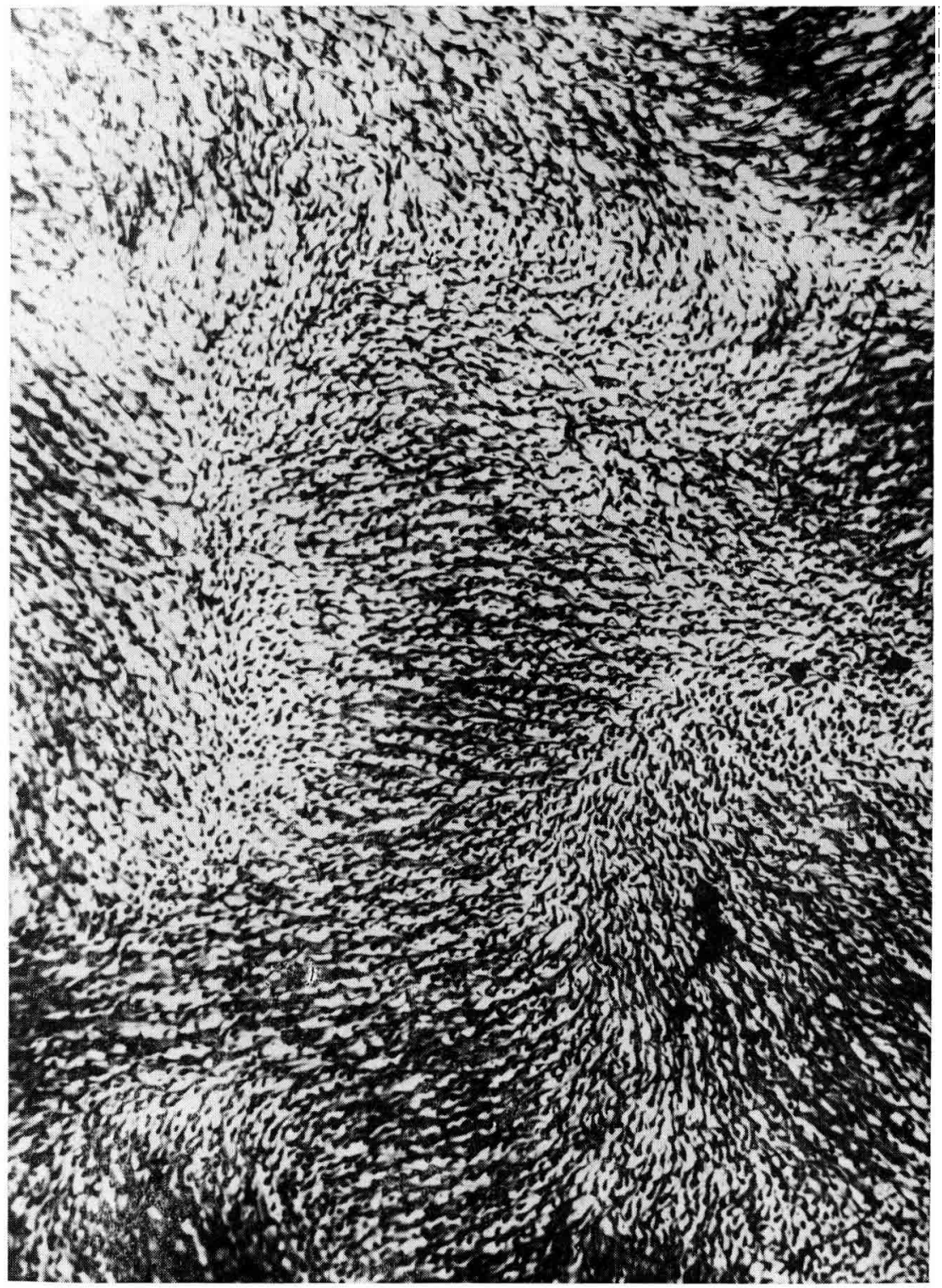

FIG. 40. - A Aneau Karakul i la naissance. Remarquer les secteurs folliculaires et leurs orientations.

jour de la vie fotale puis il se produit un mouvement inverse de sorte que les fotus de I40 jours et I50 jours sont comparables en ce qui concerne la disposition des follicules.

Ce qui les différencie, c'est la longueur et l'incurvation des poils et la forme de la boucle. A la naissance de 1'agneau, les poils ont en moyenne de 7,39 à I4,20 mm de longueur ( 17 agneaux contrôlés). On distingue plusieurs incurvations dans des plans différents le long de cha- 
que poil. Ceci est déterminé par les différents mouvements des follicules que nous venons de décrire.

Les poils émis par des groupements folliculaires voisins s'affrontent ou s'emboitent pour former des boucles. La forme de la boucle est déterminée par l'incurvation ou plutôt les incurvations des poils qui la composent. Les limites des boucles ne correspondent pas forcément à celles des secteurs folliculaires. İ̀n effet si deux secteurs voisins contribuent à la formation de la même boucle, leur limite commune se situe à l'intérieur de cette boucle. Au contraire, s'ils entrent dans la constitution de deux boucles différentes, leur limite commune se situera dans l'intervalle entre ces deux boucles.

Étant donné que chaque poil a plusieurs incurvations dans des plans différents, on ne peut pas prévoir, d'après la convergence ou la divergence des follicules, que tels secteurs roisins formeront une même boucle et tels autres entreront dans la constitution des boucles différentes. Par conséquent, mis à part quelques types très caractéristiques de boucles, telles que la boucle en cote et la boucle en rose, les dessins formés par les follicules ne reflètent pas exactement ceux formés par les boucles.

\section{b) Comparaison aVEc D'Autrfos races.}

La toison de l'agneau Bizet diffère de celle de l'agneau Karakul par plusieurs caractères dont les principaux sont les suivants :

- Ia finesse et l'homogénéité du diamètre des fibres : il n'y a pas de différence de diamètre entre les fibres primaires et secondaires : 60 p. Ioo environ des fibres ont un diamètre de 20-30 \%, 2I p. IOO entre ro et $20 \mu$ et $I_{5}$ p. roo entre 30 et $40 \%$.

- le bouclage des fibres se produit dans un plan parallèle à la surface de la peau et non dans un plan perpendiculaire comme chez le Karakul.

- le clegré de fermeture plus poussé des fibres qui forment plusieurs tours de spirale comme un ressort, anarchique.

- 1'absence de secteurs de follicules qui sont orientés de façon

Les extrémités des fibres ayant poussé avant et au début du désordre folliculaire sont incurvées. Au début de leur éruption elles ont pu s'emboiter par petits groupes pour former des petites boucles dans un plan parallèle à la surface de la peau. Ce parallélisme est vraisemblablement dû à la pression exercée par les enveloppes fœtales. La figure $4 \mathrm{I}$ montre l'accompagnement désordonné des fibres chez un fotus Bizet de $I 22$ jours dont I image folliculaire est représentée par la figure 38 .

Chez un fotus Bizet de 132 jours nous avons trouvé un bouclage de poils rappelant des boucles grossières et mal délimitées de Karakul. 


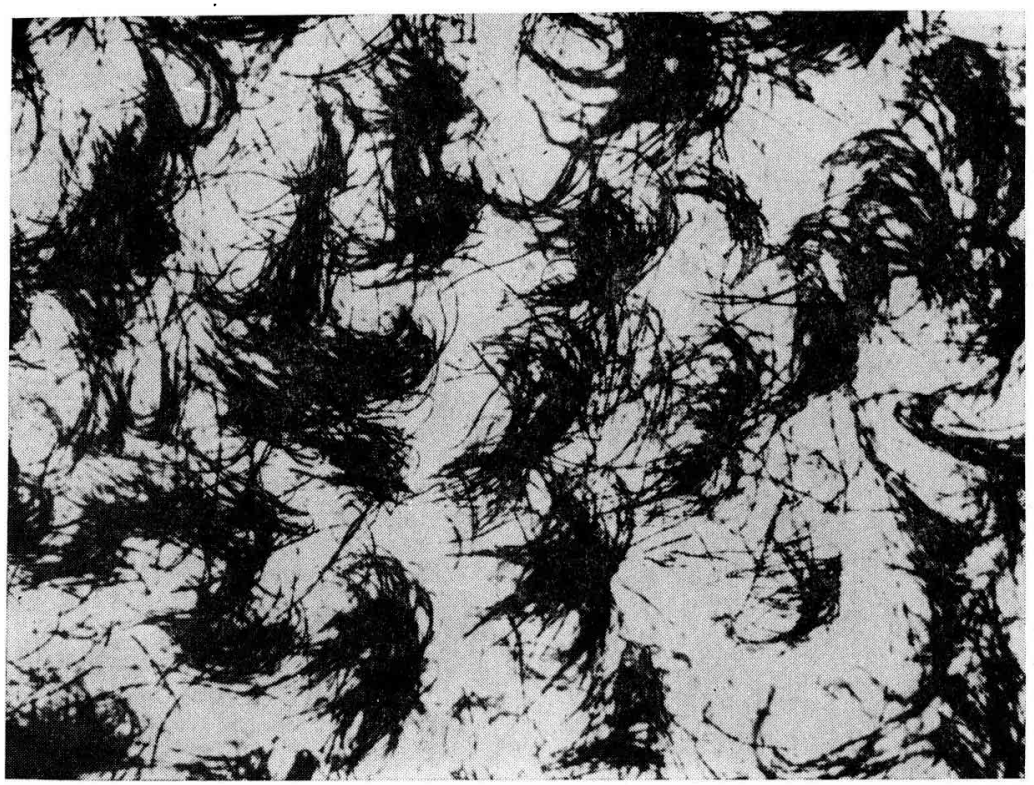

FIG. 4I. - Bouclage désordonné des fibres chez un foetus Bizet de I22 jours.

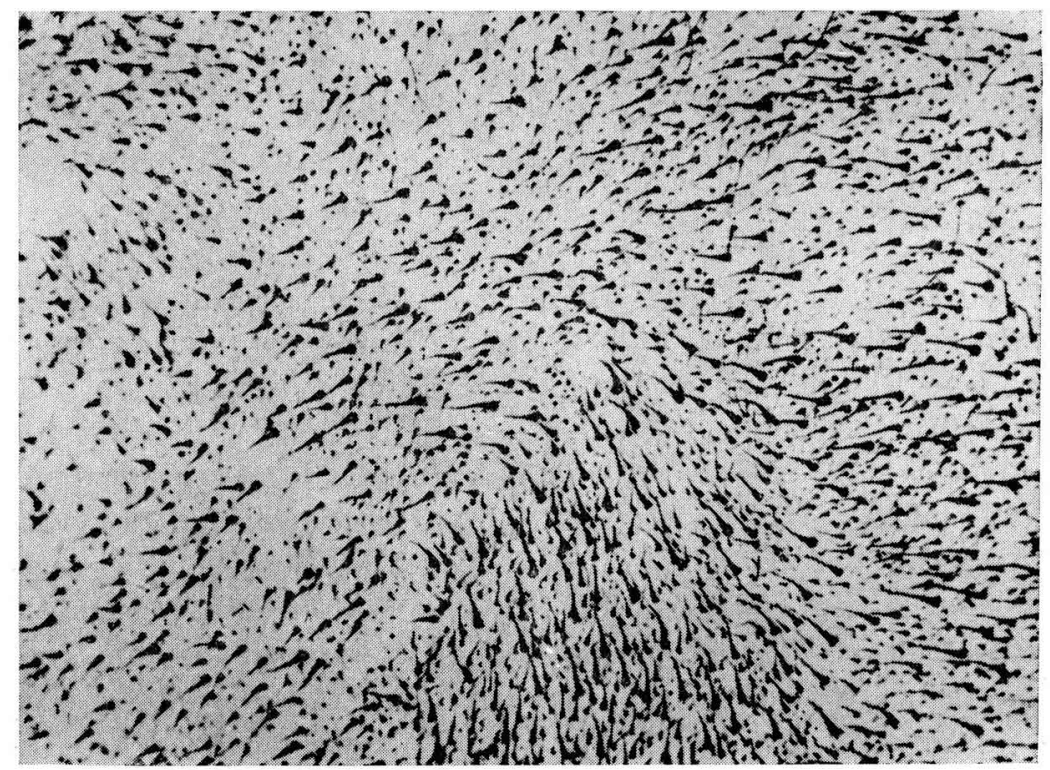

FIG. 42. - Dessin folliculaire d'un foetus Bizet de I 32 jours,

qui par hasard formait des formations bouclées ressemblant à celles du Karakul. 
Parallèlement les dispositions folliculaires (fig. 42) montraient des groupements sans toutefois présenter le parallélisme rigoureux des follicules à l'intérieur du même paquet tel qu'on le rencontre chez la race Karakul.

Chez 1'agneau de la race Limousine à toison plus grossière, on retrouve ces désordres folliculaires pour les fibres frisées tandis que les follicules produisant du jarre ont une orientation relativement uniforme.

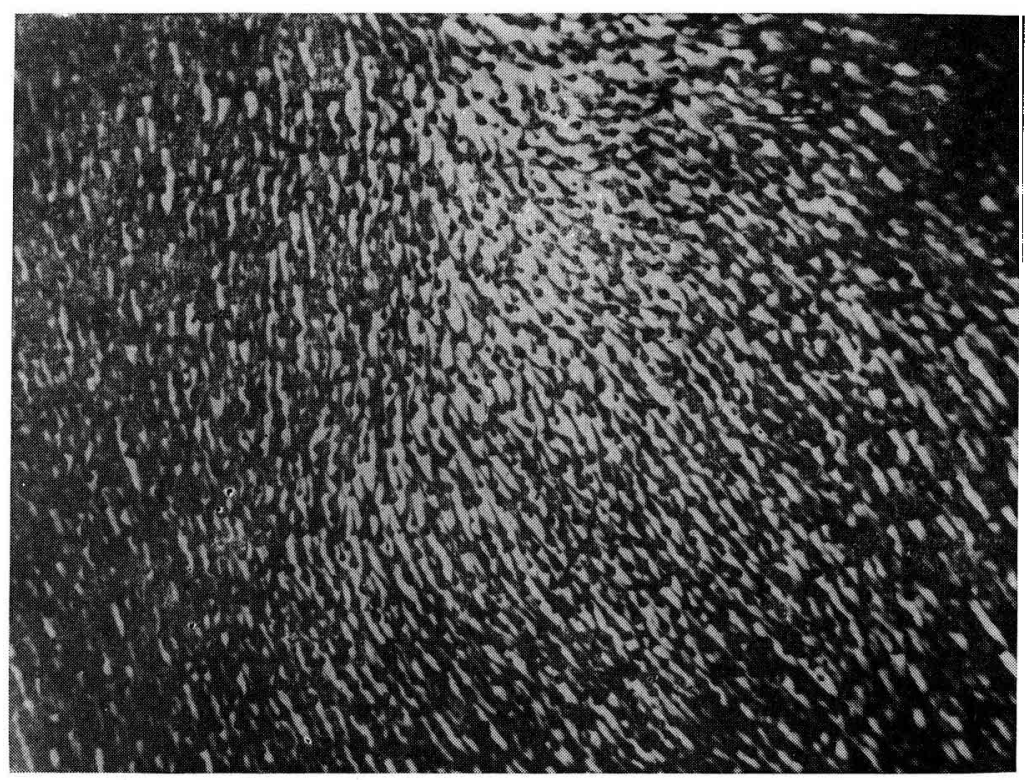

FIG. 43. - Image folliculaire d'un agneau Karakul âgé de ro jours. Les follicules ont à peu près retrouvé leur orientation unique initiale.

Il en est de même pour le croisement entre la race Karakul et la race Southdown. L'agneau croisé présente une orientation relativement unique pour les gros follicules et un désordre pour les petits qui sont de loin les plus nombreux.

Par conséquent, ce qui caractérise l'embryogenèse folliculaire du Karakul par rapport aux autres races dont les fibres prises individuellement peuvent également être incurvées, c'est l'organisation des secteurs de follicules au sein desquels les follicules restent orientés parallèlement. Quant à l'incurvation des fibres qui se produit aussi bien chez le Karakul que chez les autres races, nots en analyserons les causes ultérieurement.

\section{3) Débouclage.}

Nous avons vu qu'après le $145^{\mathrm{e}}$ jour de la vie fotale, date qui coïncide avec le maximum des remaniements folliculaires, le phénomène inverse a lieu et les groupes de follicules ont tendance à reprendre leur 
direction initiale et uniforme. Ce mouvement de retour est assez rapide de sorte qu'au Io $^{\mathrm{e}}$ jour de la vie post-natale la majorité des follicules ont à peu près retrouvé leur orientation initiale (fig. 43). Ainsi chez l'agneau âgé et chez l'adulte la direction générale des follicules pileux est la même que chez le foetus de moins de 4 mois (fig. 44 et 39).

Cependant à la naissance la forme idéale de la boucle pour la fourrure n'est pas atteinte chez tous les agneaux. Pour certains il est nécessaire d'attendre quelques jours pour obtenir un meilleur état de dévelop-

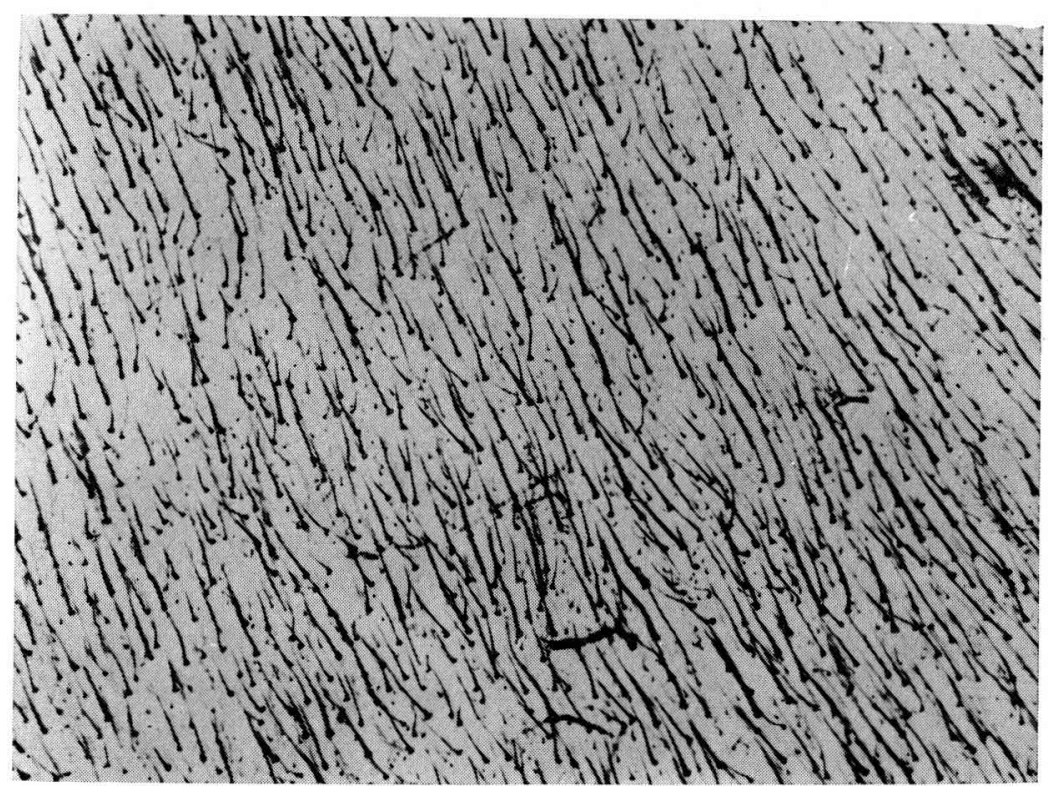

FIG. 44. - Orientation folliculaire chez une brebis Karakul adulte. Remarquer la forme rectiligne des follicules primaires et l'incurvation des follicules secondaires.

pement. On peut expliquer ces variations par des différences dans la durée de gestation. C'est ainsi que pour 37 brebis Karakul les durées de gestation que nous avons observées ont varié de 140 à $I_{52}$ jours.

Variation de la durée de gestation chez la race Karakul.

\begin{tabular}{|c|c|c|c|c|c|c|c|c|c|c|c|c|c|}
\hline Durée de gestation en jours.......... & 140 & I4I & I42 & I 43 & 144 & I 45 & 146 & I 47 & I 48 & I 49 & 150 & I 5I & I5 \\
\hline Nombre d'animaux & I & & 2 & & & 2 & I & 3 & 8 & 8 & 7 & 2 & 3 \\
\hline
\end{tabular}

Les agneaux à vie embryonnaire courte possèdent des boucles solides et susceptibles de s'améliorer durant les trois à quatre jours qui suivent, du fait de l'augmentation de la longueur des poils. Car le mouvement inverse des follicules n'est pas encore avancé. Par contre les agneaux nés après une longue période embryonnaire possèdent des 
boucles lâches en voie du débouclement rapide. Par conséquent leur sacrifice hâtif s'avère nécessaire. Le débouclement commence donc plus ou moins avant la naissance de l'agneau et se traduit par la désorganisation (état lâche) des boucles d’abord, et par leur éloignement de la sur-

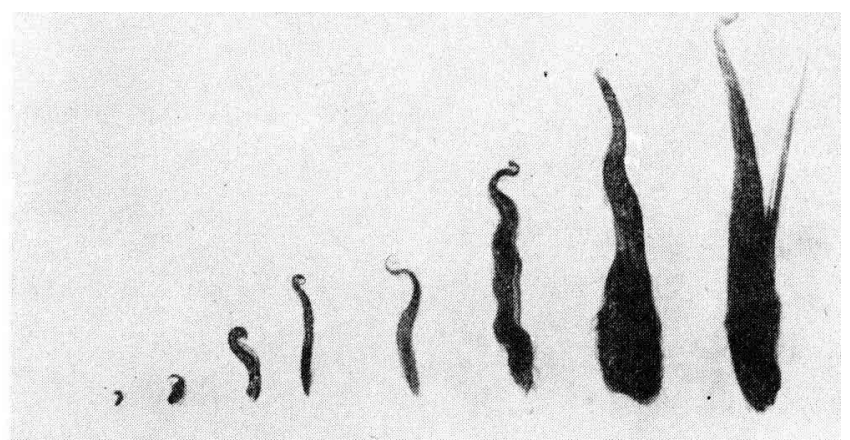

FIG. 45. - Évolution d'une mèche de la toison de l'agneau Karakul durant la première année (I - 365 jours). La boucle se transforme en mèche dont l'extrémité reste toujours bouclée jusqu'à la premiere tonte.

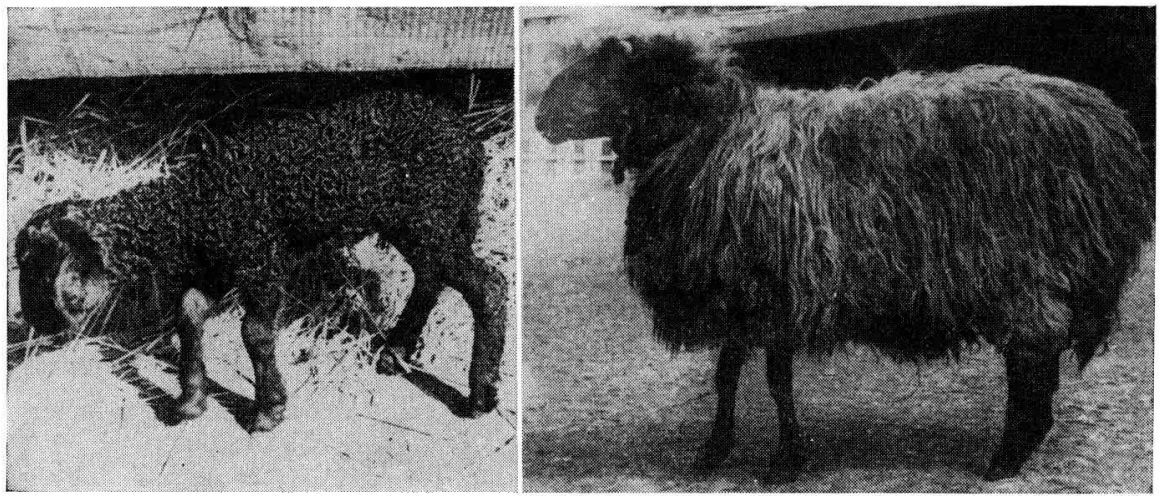

Fig. 46. -- Agneau Karakul à la naissance. Les boucles ont leurs formes définitives.
Fig. 47. - Bélier Karakul. Il n'y a plus aucune trace de boucle.

face de la peau ensuite. En effet le poil croît après la naissance d'une façon rectiligne.

I1 est également possible que les variations individuelles enregistrées à la naissance traduisent les variations dans le temps de réorganisation folliculaire pendant la vie fœale.

Ainsi la boucle ne se déroule pas mais se transforme en une mèche 
dont l'extrémité bouclée s'éloigne de la peau. En général et malgré les intempéries, l'extrémité de la mèche reste bouclée chez l'animal vivant jusqu'à un an et plus si la tonte n'intervient pas. La figure 45 montre cette évolution de la boucle depuis la naissance de l'agneau jusqu'à l'âge d'un an.

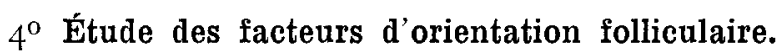

L'orientation de follicules par secteurs implique l'existence de facteurs externes et localisés agissant ensemble sur les groupements de follicules.

Nous avons recherché si des modifications structurales de la peau ou une croissance hétérogène pouvaient offrir tune explication satisfaisante.

Nous nous sommes proposés de voir s'il se produit des modifications dans la forme, la quantité et la disposition des fibres élastiques et le collagène. Des échantillons de peau d'environ $2 \mathrm{dm}^{2}$ de surface prélevés sur les zones limites des secteurs de follicules ont été utilisés à cet effet. Les fibres élastiques existent dans la peau du Karakul au niveau du stratum intermedium (couche située entre les glandes sébacées et la jonction du muscle arrecteur du follicule). Les coupes d'épaisseur 5 à $20 \mu$ n'ont montré aucune disposition particulière des fibres élastiques se rapportant aux orientations différentes des follicules. Car cette disposition est la même chez le fotus de I20, I30, I40 jours que chez l'agneau et l'adulte. Il en est de même pour le collagène.

En ce qui concerne l'hypothèse de la croissance hétérogène de la peau, nous avons essayé de voir si des modifications locales dans la densité folliculaire pouvaient la justifier. En effet, étant donné que la formation des follicules primaires s'achève bien avant les mouvements folliculaires, on devrait trouver des densités différentes pour les secteurs folliculaires et pour les zones intermédiaires. Encore faut-il que cette densité soit uniforme avant 1'apparition des changements d'orientation des follicules. Les comptages ont été faits au niveau des glandes sébacées et par projection. Nous avons trouvé les valeurs suivantes se rapportant au $\mathrm{mm}^{2}$ de la coupe et sur des aires immédiatement voisines :

Foetus de x21 jours: 30, 32, 32, 38, 33, 39, 37, 32, 3x, 32, 31, 32, 31. Densité moyenne 33,07 , écart type de la moyenne : 0,84 .

Coefficient de variation : 9, I3 p. Ioo.

Foetus de 132 jours: 32, 34, 32, 3I, 35, 31, 29, 30, 30, 34, 34.

Densité moyenne : 32 , écart type de la moyenne : 0,60 .

Coefficient de variation : $6,25 \mathrm{p}$. Ioo.

Fotus de I42 jours : 25, 28, 28, 29, 28, 3I, 27, 26, 29.

Densité moyenne : 27,88 , écart type de la moyenne : 0,64 . 
Coefficient de variation : 6,89 p. Ioo.

A gneau à la naissance: 20, 2I, I8, 2I, I9, I8, I7, I8, I7, I7, I7, 23 , 2I, I9.

Densité moyenne : I9, écart type de la moyenne : $0,5 \mathrm{r}$.

Coefficient de variation : ro, Io p. Ioo.

Karakul adulte: 4, 3, 4, 4, 5, 4, 5, 4, 5, 4, 3, 4, 4, 3, 4, 4, 4, 4, 5, 5 .

Densité moyenne : 4,I écart type de la moyenne o,I4.

Coefficient de variation : $\mathrm{r}_{5}, 6 \mathrm{I}$ p. Ioo.

Ces comptages portent uniquement sur les follicules primaires.

S'il y avait une différence de croissance sur des zones voisines de la peau au moment des mouvements des follicules et si cette différence pouvait être reflétée par la densité folliculaire, on aurait dû trouver une variation du même ordre de grandeur pour le fœtus de I2I jours et pour l'adulte, et des variations plus marquées chez les fœtus de I 32 et I 42 jours et chez l'agneau à la naissance. Or les valeurs trouvées ci-dessus montrent à peu près la même variation de la densité folliculaire avant, pendant et après les changements d'orientation des follicules. Par conséquent on ne peut pas conclure à une apparition des différences de croissance dans la peau au moment des mouvements folliculaires chez les fœtus de I25 à I50 jours; si un tel phénomène a lieu, il n'est pas décelable par la détermination de la densité folliculaire.

Pour étudier l'influence de l'orientation des follicules sur le comportement des poils chez l'agneau âgé et chez l'adulte nous avons provoqué expérimentalement un remaniement des follicules en changeant leur direction par des greffes. Les résultats ont été les suivants :

Tandis que les homo et les syngénésiogreffes ont été rejetées après ro jours et 25 jours respectivement, les autogreffes ont réussi.

D'une façon générale les greffons diminuent de taille. Leurs surfaces se réduisent de 3 à 5 fois au début puis augmentent à nouveau.

Durant les 15 à 20 premiers jours, la partie kératinisée du poil tombe. Le bulbe se résorbe en partie ainsi que les pigments qu'il contient.

Ia régénération des follicules suit la chute des poils. Les nouvelles fibres ainsi formées se distinguent des anciennes par trois caractères :

- pigmentation plus intense,

- diamètre plus fort,

- densité plus grande.

L'augmentation de la densité pileuse s'explique par la réduction de surface du greffon.

Les follicules secondaires se régénèrent de la mêtne façon que les primaires.

Dans certaines parties du greffon il n'y a pas de régénération de follicules.

Dans certains cas il y a formation de croûte à la surface du greffon, 
ce qui provoque un retard à tous les processus de chute et de régénération qui ont lieu également sur le porte-greffe dans la zone de cicatrisation. Cette zone se repère par l'apparition de quelques poils blancs.

L'orientation des follicules du greffon est restée identique à celle que nous leur avons imposée au moment de la greffe. Ia forme des poils émis du greffon est la même que ceux du porte-greffe qui sont plus ou moins onduleux. Chez l'animal jeune (moins d'un mois) les poils émis du greffon s'accompagnent les uns les autres pour former une boucle grossière. Chez l'adulte il n'y a pas d'accompagnement aboutissant à la formation de la boucle de sorte qu'après deux à trois mois l'endroit greffé devient difficile à reconnaître. Cependant les poils des greffons sont plus courts et leur orientation reste différente de celle des poils du porte-greffe.

Mais on ne peut tirer de conclusions valables du comportement des fibres dans le greffon car, les fibres qui repoussent sont d'un diamètre beaucoup plus élevé et l'affrontement avec les fibres du porte-greffe ne correspond plus à ce qui se produit chez l'embryon, où les fibres qui s'affrontent sont de même taille et par conséquent de résistance sensiblement égale.

\section{B. - Facteurs d'incurvation et de frisure des poils.}

Nous avons vu que la formation de la boucle pouvait en partie s'expliquer par l'orientation des différents secteurs de follicules. Un problème cependant demeure sans réponse. Pourquoi les poils s'incurvent-i1s pendant la vie fotale permettant ainsi la formation de boucles très régulières an lieu de simples épis sur les lignes d'affrontement.

Le résumé bibliographique que nous avons donné a mis en évidence les différentes interprétations qui ont été suggérées pour expliquer l'incurvation et la frisure. I.es hypothèses étant nombreuses nous avons été amenés à les vérifier et à les discuter dans le cas qui nous intéresse.

Ces expériences et leurs résultats font l'objet des pages suivantes.

Les facteurs susceptibles d'intervenir dans l'incurvation et la frisure des fibres sont les suivants :

- influence de la morphologie de la fibre,

- influence de la morphologie du follicule pileux,

- influence du fonctionnement du follicule pileux,

- influence des enveloppes fœtales.

\section{$I^{0}$ Influence de la morphologie de la fibre.}

a) SECTION DU POIn : La forme et la taille de la section droite du poil ont souvent été tenues pour un des facteurs responsables de l'incurvation et de la frisure du poil.

Les sections droites des poils que nous avons observées aussi bien à 


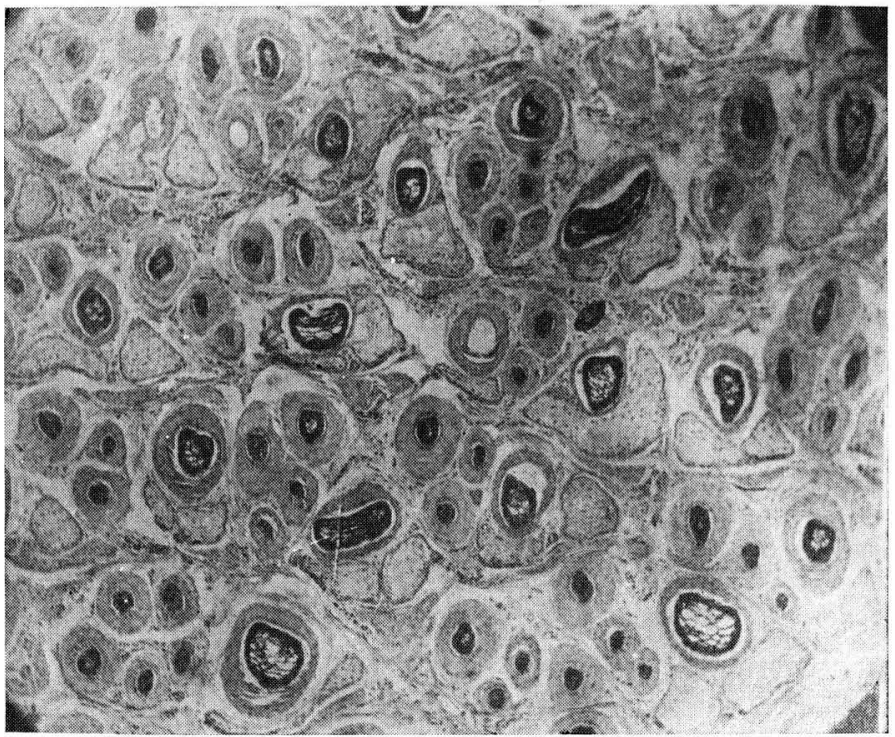

Fig. 48. -- Queleques aspects de la forme de la section droite des fibres chez l'agneau Karakul.

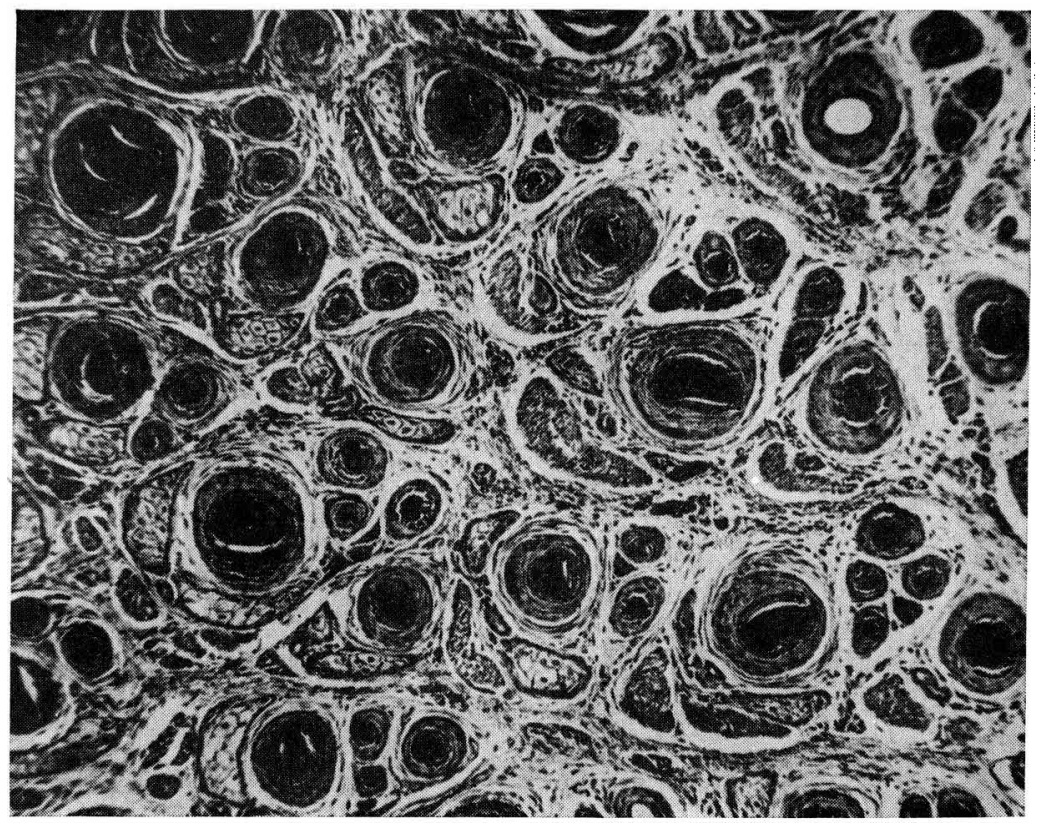

FIG. 49. - Quelques aspects de la forme de la section droite des fibres chez l'agncau Karakul. 
l'intérieur de la peau qu'à l'extérieur de celle-ci ont des formes diverses : elles peuvent être rondes, ovales, triangulaires, en croissant, polygonales ou aplaties latéralement (fig. 48 et 49 ) ce qui laisse supposer que la forme de la section transversale ne joue aucun rôle. D'ailleurs chez l'adulte dont les poils ne sont plus incurvés, bien que le polymorphisme s'atténue avec prédominance des formes rondes et ovales, ces différentes formes se retrouvent.

b) LA MOELLE : La présence de la moelle dans la fibre n'a aucune relation avec l'incurvation du poil. En effet parmi les agneaux à toisons

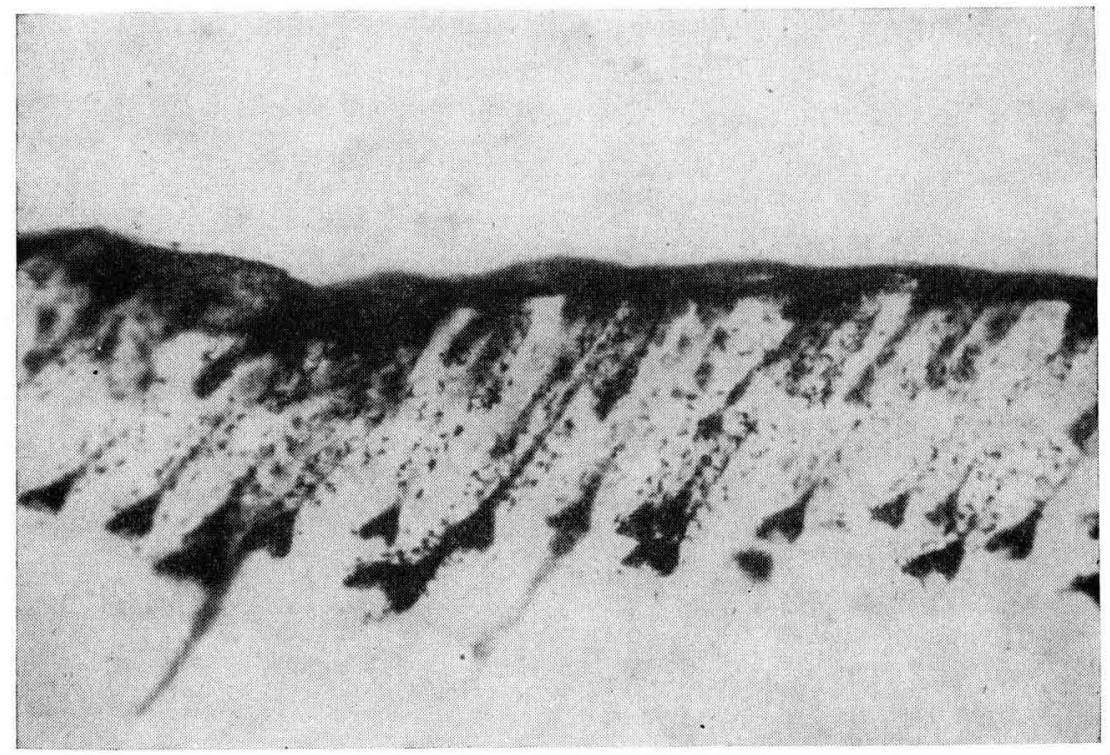

FIG. 5०. - Fœtus Karakul de 85 jours (tête). Les follicules ont une forme rectiligne.

également bouclées, certains possèdent des poils médullés, d'autres non médullés et d'autres enfin chez qui des poils médullés et non médullés se présentent en proportions diverses (tableau I2).

La toison de l'agneau de la race Limousine présente les mêmes caractères de médullation, elle est pourtant composée de fibres droites.

Cependant la moelle a une certaine influence sur le degré de fermeture de la boucle, le pourcentage des poils médullés étant plus faible pour les petites boucles que pour les grosses boucles ainsi que le montre la figure 59 .

\section{$2^{\circ}$ Influence de la morphologie du follicule.}

Le follicule pileux, au début de son développement est incliné par rapport à la surface de la peau, mais il a une forme rectiligne (fig. 50). 
Cette disposition du follicule reste inchangée jusqu'au stade de la différenciation des fibres.

A cet âge, le bulbe $d u$ follicule semble être entraîné latéralement. Il en résulte la formation d'un coude immédiatement au-dessus du bulbe. C'est précisément le niveau le moins résistant du follicule. En effet, d'une part le bulbe qui est assez volumineux et d'autre part la tige du follicule qui se kératinise à cet âge se prêtent moins facilement aux déformations.

I a présence de ce coude n'a pas d'influence directe sur l'incurvation de la fibre car elle existe même chez les follicules producteurs de fibres droites. Nous l'avons constaté également à l'aisselle d'un agneau à la naissance alors qu'à cet endroit les poils poussent presque droits. En outre, selon Auber (I95I) les follicules en forme de "stick de hockey " de souris, de rats et de certains chiens, produisent des fibres droites.

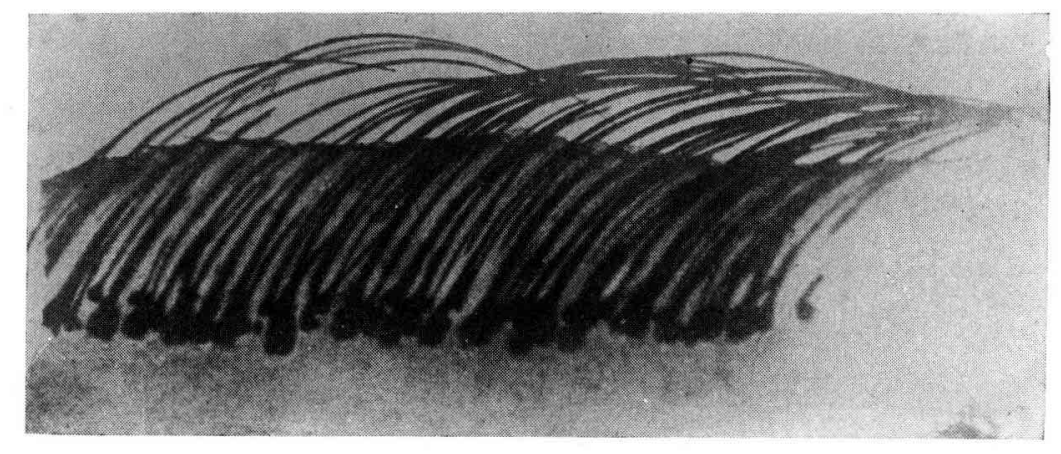

lis. 51. - Forus Karakul de I 2 jours (flanc). Remarquer la forme incurvée des follicules et celle des libres.

I a présence du coude à la base du follicule semble être une conséquence de la différence de structure dans les différentes couches de la peau.

Iin effet an niveau de la base des follicules on observe une densité plus forte de fibres collagènes entrelacées autour des bulbes. Ceci est un fait commun à toutes les races ovines, selon nos propres observations. Cependant cliez le foetus Karakul, à la différence des autres races ovines que nous avons étudiées les coudes sont orientés du même côté, ce qui pourrait être en relation avec une certaine orientation dans la structure de la peau.

Plus tard, entre le $\operatorname{IO5}^{\mathrm{e}}$ et le $\operatorname{II} 5^{\mathrm{e}}$ jour de la vie embryonnaire les tiges des follicules s'incurvent légèrement (fig. 5I). Cette incurvation du follicule coïncide avec le début de la kératinisation de la fibre qui, conme nous le verrons plus 1oin, peut provoquer l'incurvation de la fibre. I'autre part le follicule se redresse à l'âge de I30 à I35 jours ; il en est de même pour la partie intrafolliculaire de la fibre. 
Chez l'agneau âgé et l'adulte Karakul, les poils sont droits ou légèrement onduleux et proviennent des follicules eux-mêmes droits. Par contre la laine du Karakul adulte, composée de fibres frisées est issue des follicules secondaires qui sont incurvés. On peut donc conclure que :

- Les follicules droits produisent des fibres droites.

- Les follicules incurvés émettent des fibres incurvées ou frisées.

Ces deux principes sont valables pour toutes les races ovines.

Par conséquent l'incurvation de la fibre et celle du follicule sont liées l'une à l'autre mais il est difficile de dire laquelle des deux entraîne l'incurvation de l'ensemble.

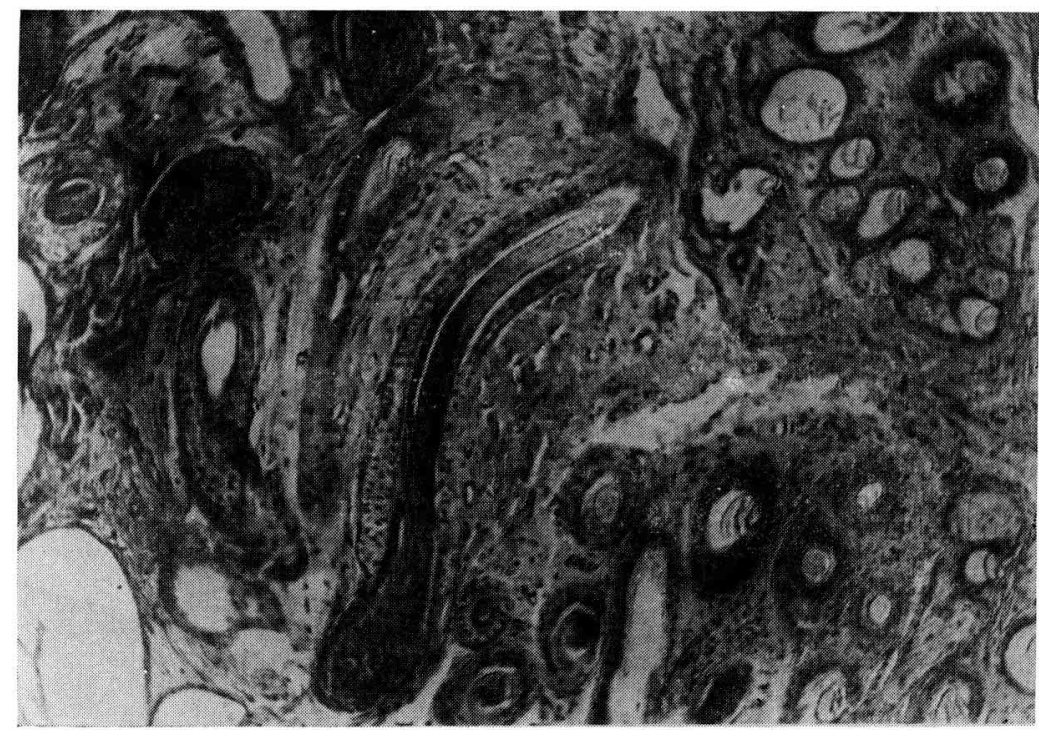

I'IG. 52. - Brebis Ile-de-France. Coupe longitudinale d'un follicule montrant la kératinisation de la fibre du côté concave de la courbure du follicule.

\section{$3^{\circ}$ Influence $\mathrm{du}$ fonctionnement $\mathrm{du}$ follicule.}

a) Kúratinisation. - I'examen des coupes longitudinales du follicule montre que la kératinisation de la fibre et de la gaine épithéliale interne a lieu d'abord sur le côté concave du follicule (fig. 52).

Cette courbure peut être la conséquence de la kératinisation car elle se produit après le phénomène de kératinisation. AstBury (I942) a démontré qu'au cours de la kératinisation les structures fibrillaires se contractent. Cette contraction étant unilatérale du moins au début de la kératinisation, tend à fléchir la fibre et le follicule vers un côté qui est celui du muscle arrecteur. Notons par ailleurs que les gaines épithéliales du follicule sont plus minces de ce même côté.

I,es races ovines que nous avons étudiées présentent toutes des fibres 
plus ou moins incurvées ou frisées [(Karakul, Bizet, Ile-de-France, Mérinos, Limousine). La race Iimousine réputée par sa toison raide, présente également à la naissance des poils légèrement incurvés et de la laine frisée; le jarre lui-même est légèrement ondulé. Or le début de la kératinisation est unilatérale chez toutes ces races ; d'où la relation entre la kératinisation et l'incurvation de la fibre. Mais chez le Karakul adulte les poils poussent droits bien que le mode de kératinisation reste le même. Il se peut qu'un autre facteur intervienne pour expliquer ce dernier cas et dont la nature nous échappe.

b) Structure hemicylindrioue de la fibre. - Les brins de laine de la race Mérinos comportent, comme 1'ont montré de nombreux auteurs, une structure hémicylindrique, constituée par l'orthocortex et le paracortex (voir historique). Une structure analogte a été constatée par KASSENBECK (1956) chez les fibres de cellulose régénérée qui à l'inverse des fibres de cellulose native, présentent une certaine ondulation lors du mouillage dans l'eau.

Cette structure est due au fait que ces fibres sont, en réalité des filaments conjugués lors de la filature, chaque moitié ayant été traitée différemment.

Dans les deux cas (laine et cellulose régénérée) la structure hémicylindrique a été supposée comme responsable de la frisure.

Nous avons cherché à savoir si cette structure se retrouve dans les fibres du Karakul.

Pour cela nous avons utilisé les colorations différentielles par le vert Janus (Horio et Kondo I953) le bleu de méthylène (Leveau 1953) ainsi que d'autres colorations basiques; safranine, fuchsine basique, bleu polychrome. La mise au point du mode d'utilisation de ces colorants a été effectuée sur des laines différentes : Mérinos, Ile-de-Firance et Limousin.

Tous les brins de laine de ces trois races ont montré la structure hémicylindrique, spécialement après coloration par le bleu polychrome après traitement à l'acide picrique (Rougeot I956).

En ce qui concerne la race Karakul, ces techniques ne sont pas directement applicables par suite de la présence du pigment qui pourrait masquer la réaction. Nous avons donc été amenés à décolorer les fibres de 1'agneau et de l'adulte Karakul par l'eau oxygénée (à 50 volumes). Ce traitement ne modifiant pas la mise en évidence de 1'ortho et du paracortex comme nous avions pu nous en assurer en faisant subir le même traitement à de la laine Mérinos.

D'autre part, nous avons également utilisé les fibres blanches des variétés grise et " abrache » de Karakul ainsi que des fibres naturellement depigmentées de Karakul noir adulte ( $\left.{ }^{1}\right)$.

(I) La toison du Karakul se dépigmente avec l'âge, de sorte que chez l'adulte on trouve une certaine proportion de fibres blanches. 
La présence de l'ortho et du paracortex a été mise en évidence chez 1a laine Karakul alors que les poils n'ont présenté une telle structure ni sur des coupes transversales minces ni sur la fibre entière.

Nous avons remarqué par ailleurs que l'ortho et le paracortex sont d'autant plus visibles que la laine est plus frisée.

Nous confirmons donc la relation entre la structure hémicylindrique de la laine et la frisure de celle-ci, ce qui existe également chez les fibres courtes et fines de l'agneau Karakul, en réalité la laine. Mais les poils qui constituent la boucle chez l'agneau Karakul ne présentent pas de structure hémicylindrique visible.

\section{$4^{\circ}$ Influence des enveloppes fœtales.}

La pression exercée par les enveloppes fœtales est susceptible de jouer un certain rôle dans le phénomène de l'incurvation des poils chez le foetus Karaku1.

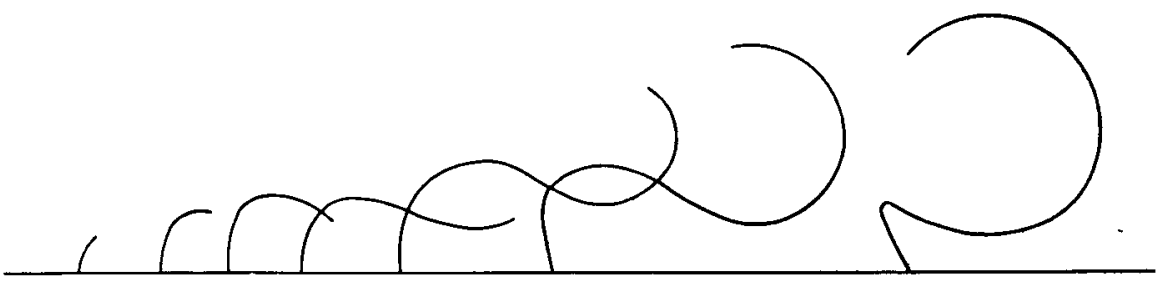

FIG. 53. - Schéma montrant l'évolution de l'incurvation des poils chez le foetus Karakul depuis la sortie des fibres à la surface de la peau, jusqu'à la naissance de l'agneau.

Cette remarque est valable pour d'autres races ovines dont les agneaux présentent à la naissance des boucles disparates, mais la nature et le diamètre du poil dans la race Karakul interviendraient ainsi que l'angle d'implantation des follicules dans la peau pour expliquer l'incurvation régulière du poil (fig. 53).

\section{DISCUSSION}

L'analyse de la boucle de l'agneau Karakul montre qu'elle dépend de deux facteurs : l'incurvation simple et générale des poils pendant la vie embryonnaire, ce qui implique l'absence de frisure de ces poils, et la réorganisation, par groupe, pendant une partie strictement limitée de la vie foetale, de l'orientation des follicules qui, jusqu'au $\mathrm{II}^{\mathrm{e}}$ jour, étaient restés parallèles entre eux sur presque tout le corps.

L'examen des sections transversales ne nous a permis d'établir aucun lien entre la forme de ces sections et l'incurvation contrairement aux observations de Brown (1853) chez les moutons et de FriTsCH (I92I) chez l'homme, puisque le premier n'a pas tenu compte des autres 
formes de la section que les formes ovales et que le second a confondu l'incurvation et le bouclage.

Au contraire, le rôle de la forme des follicules mérite d'être discuté. C'est PFEIFEr (I928) qui a tenu la présence du coude à la base du follicule comme responsable de la formation de la boucle.

En reconstituant l'expérience de PFEIFER, nous nous sommes aperçus que si le fil métallique n'était pas complètement redressé après l'incurvation produite au départ par le coude, c'était dû en grande partie à ce que le fil ne remplissait pas totalement le canal du tube de verre.

En outre, nous avons constaté l'existence de coudes dans les follicules à 1'aisselle d'un agneau, or à cet endroit il n'y a jamais de boucle.

D'autre part, les follicules de Souris et de Chien, tout en étant coudés, produisent des fibres droites (AUBER I95I). Il en est de même chez le Karakul après la naissance; ce que PFEIFER prétend expliquer en admettant alors l'existence d'un deuxième coude d'effet antagoniste à un niveau plus élevé. Nous ne l'avons pas observé, soit chez le foetus à terme, soit chez l'agneau.

La forme incurvée du follicule, contrairement à l'opinion de TänzER (I928) ne peut pas expliquer le bouclage des poils, bien qu'il y ait une relation évidente entre l'incurvation du poil et celle du follicule, car on constate chez le foetus Karakul que le poil s'incurve du côté où la kératinisation est la plus précoce (kératinisation unilatérale, voir ATsBury, I942 et AUBER I95I), ce qui laisserait supposer que c'est la courbure de la fibre qui entraine celle du follicule; mais on est également obligé de tenir compte du fait que dès la fin de la vie embryonnaire, le mode de kératinisation reste inchangé alors que les follicules primaires se redressent et que les fibres poussent droites. Ainsi on ne peut préciser celui des 2 éléments (follicule ou fibre) qui entraîne la courbure de l'ensemble.

Le redressement des follicules primaires à la fin de la vie embryonnaire ne peut être provoqué, comme le supposait TäNzER, par l'augmentation de l'épaisseur de la peau, puisque les follicules secondaires restent toujours incurvés même chez l'adulte.

La structure hémicylindrique de la laine, reconnue par Horio et Kondo (I953) comme responsable de la frisure de la laine, parait jouer le même rôle chez le Karakul dans les fibres secondaires qui sont frisées, mais nous n'avons pu la mettre en évidence dans les fibres primaires qui sont simplement courbes.

Ainsi, tout en vérifiant l'exactitude de l'hypothèse de Horio et Kondo, nous pouvons affirmer que la structure hémicylindrique n'est pas responsable de la courbure transitoire des fibres primaires du Karakul.

L'évolution de la morphologie des fibres à la fin de la vie fotale et au début de la vie post-natale nous a conduit à penser que la nature des fibres, leur densité, l'angle d'inclinaison des follicules dans la peau, doi- 
vent être des facteurs essentiels de l'incurvation dans les conditions de la vie fotale (liquide amniotique, température, pression des enveloppes..).

Les auteurs qui avaient déjà reconnu l'organisation des follicules

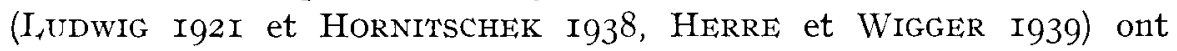
essayé de l'expliquer par des'croissances inégales de la peau. L'analyse de la densité folliculaire au cours de la période de remaniement ne nous a pas permis de déceler des variations locales de croissance.

Nous avons cherché si des modifications dans l'orientation ou la densité des fibres élastiques et collagènes pouvaient expliquer les réorientations folliculaires.Nous n'avons pu trouver de variation permettant de lier les mouvements de follicules à des variations structurales de la peau.

Le travail d'HoRNITSCHEK (I938) a bien mis en évidence les relations existant d'une part entre le dessin formé par les boucles et le type auquel elles appartiennent et d'autre part, le dessin d'ensemble des follicules. Notre étude a apporté 4 éléments essentiels à 1'observation d'HorNiTSCHEK :

$I^{0}$ Lors de leur formation, les follicules sont parallèles entre eux sur toute la surface du corps, jusqu'à la fin du $4^{\mathrm{e}}$ mois.

$2^{\circ}$ Le dessin d'ensemble des follicules observé à la naissance, provient de ce qu'il se forme des secteurs au sein desquels les follicules restent parallèles entre eux, mais présentent des orientations différentes de celles des follicules de secteurs voisins.

$3^{0}$ Après la naissance le bouclage s'efface et simultanément tous les follicules reprennent une orientation parallèle.

$4^{\circ}$ Chez les races Mérinos, Ile-de-France, I,imousine et Bizet qui ne présentent jamais à la naissance que des petites formations bouclées ne formant aucun dessin, on ne rencontre pas d'organisation folliculaire prénatale comparable à celle du Karakul.

Par conséquent en présence d'un parallélisme aussi net entre le dessin des boucles et l'organisation des follicules on doit conclure que le bouclage du Karakul est dû à une organisation folliculaire transitoire qui lui est propre.

\section{CONCLUSION}

Les boucles de l'agneau Karakul à la naissance sont constituées uniquement par les fibres issues des follicules primaires.

I a formation de la boucle n'est pas due simplement, comme l'ont pensé la plupart des auteurs, à l'incurvation des poils, mais également à leur accompagnement régulier par paquets pour former une boucle. On a pu constater que l'incurvation de la fibre était associée à la courbure du follicule. Mais, dans l'état actuel de nos connaissances on ne peut pré- 
ciser celui des deux éléments (fibre ou follicule) qui entraîne la courbure de l'ensemble. Ainsi la structure hémicylindrique de la laine qui est responsable de la frisure de celle-ci, n'a pas pu être mise en évidence dans le poil de l'agneau Karakul qui est simplement incurvé, et ne peut donc être invoquée comme cause de cette incurvation.

De même, la kératinisation unilatérale du poil en formation, n'est pas toujours associée à la production des poils incurvés.

Le bouclage proprement dit, c'est-à-dire l'accompagnement régulier des poils pour former une boucle est provoqué, à la fin de la vie embryonnaire, par l'apparition progressive, dans la peau, de secteurs au sein desquels les follicules restent parallèles entre eux, mais présentent des orientations différentes de celles des follicules de secteurs voisins. Après la naissance tous les follicules reprennent leur parallélisme initial. Ce mouvement qui est accompagné du redressement de l'incurvation des follicules provoque la dislocation des boucles qui deviennent de plus en plus lâches et pédonculées, les fibres poussant droites. Cependant les boucles formées pendant la vie embryonnaire se maintiennent à l'extrémité des fibres à mesure que la fibre croît, s'éloignant progressivement de la surface de la peau.

Nos observations chez d'autres races ovines où n'existe chez l'agneau nouveau-né aucun dessin formé par des boucles, ont montré qu'à partir du $120^{\mathrm{e}}$ jour de la vie fœetale jusqu'à l'âge adulte, une véritable anarchie s'installe dans l'organisation de leurs follicules.

La beauté de la fourrure de l'agneau Karakul est donc le résultat d'un ensemble de phénomènes, que l'on ne retrouve pas chez les autres races, à savoir :

- la participation pratiquement exclusive des fibres primaires non frisées dans la constitution des boucles;

- la pousse incurvée de ces poils pendant la vie fœtale :

- l'organisation transitoire, entre le $\mathrm{I}_{20} \mathrm{e}$ jour et la naissance des follicules en petites unités à orientation différente.

\section{DEUXIÈME: PAR'TIF;}

\section{FACTEURS LIÉS AUX DIMENSIONS DE LA BOUCLE ET A SA QUALITÉ}

Par qualité de la boucle, on entend la fermeté du bouclage, sa régularité et sa netteté. Bien entendu il s'agit de boucle en haricot et en tube, puisqu'elles constituent les meilleurs types de boucle. Mais la qualité est essentiellement une appréciation subjective. Or on sait que les grosses boucles sont en majorité de qualité médiocre, aussi avons-nous recherché les corrélations pouvant exister entre les caractéristiques de la peau de la 
toison ou des fibres et la taille de la boucle. Nous avons adopté la classification suivante :

Petite boucle : largeur inférieure à $4,9 \mathrm{~mm}$ (fig. 54).

Boucle moyenne : largeur : 4,9-7 mm (fig. 55).

Grosse boucle : largeur : 7,I-Io mm (fig. 56).

Type Tchakmaki : largeur supérieure à Io mm (fig. 64).

Incidemment, nous avons été amenés à envisager le problème de la qualité, les moyennes et petites boucles n'étant pas nécessairement de très bonne qualité.

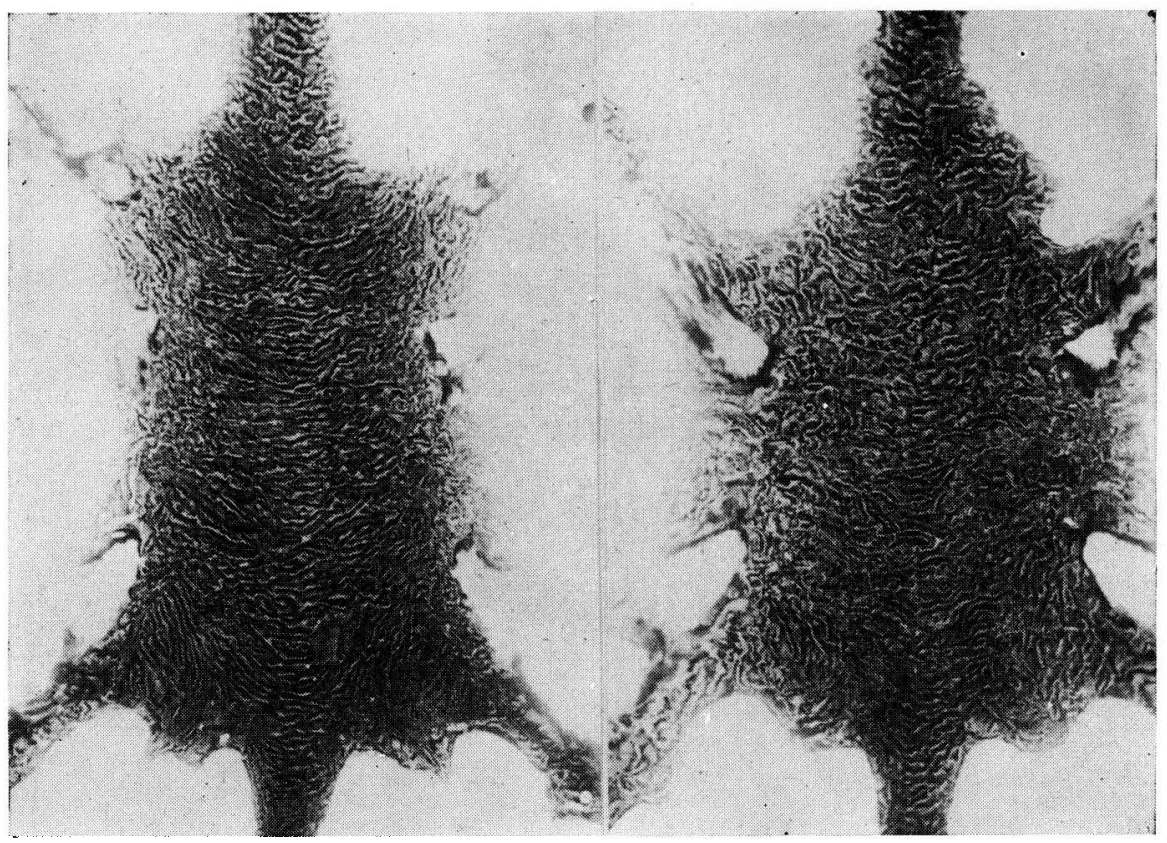

Frg. 54. - Peau d'agneau Karakul. Type à petites boucles. Remarquer le champ défectueux

Fig. 55. - Peau d'agneau sur la croupe.

Karakul. Type à boucles moyennes.

Notons que la distinction entre les 3 catégories de boucle ne peut se faire nettement que sur des boucles de bonne qualité.

Nous avons étudié les caractéristiques suivantes de la toison des fibres et de la peau.

\section{Io Pourcentage de duvet $\left(^{1}\right)$.}

Le pourcentage de duvet a été examiné d'après 1'histogramme des longueurs des fibres, sur I7 peaux tannées d'agneau Karakul d’origine afghane.

(I) Ce terme couramment utilisé en pelleterie comprend l'ensemble des fibres secondaires qui constituent la laine chez le Karakul telle que nous l'avons définie précédemment. Nous avons donc employé le terme duvet uniquement à propos des aspects pelletiers de la fourrure. Partout ailleurs il est remplacé par le terme de fibre secondaire. 
Ces peaux ont été classées selon la grosseur de leur boucle en quatre catégories. Les valeurs obtenues pour le pourcentage de duvet sont indiquées dans le tableau $\mathrm{X}$.

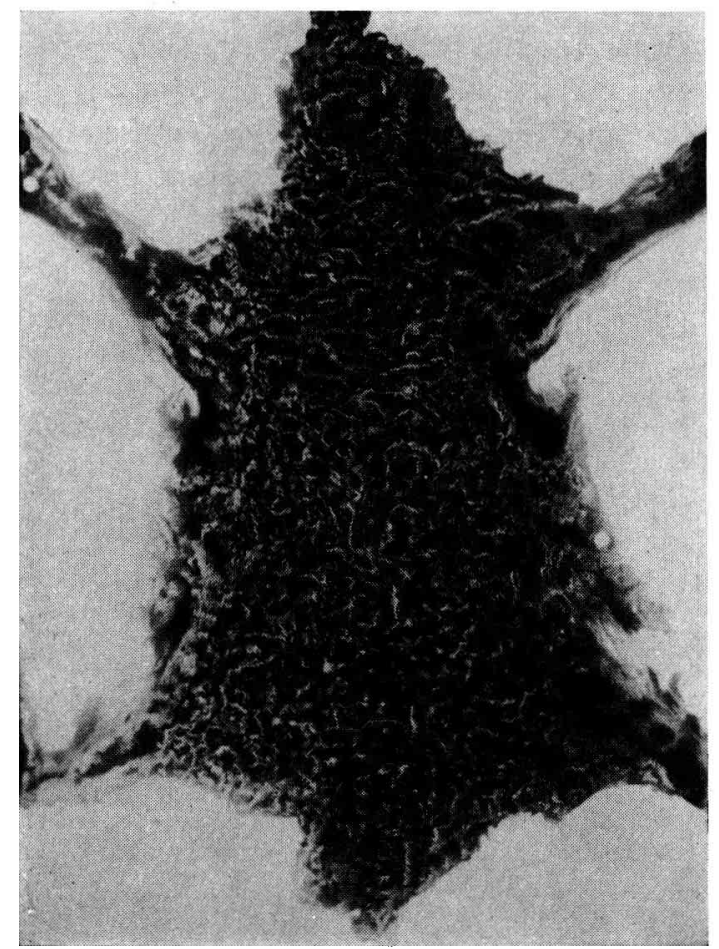

Fig. 56. - Peau d'agneau Karakul. Type à grosses boucles.

TAbleau $X$

Relation entre la grosseur de la boucle (en $\mathrm{mm}$ ) et le pourcentage de duvet.

\begin{tabular}{|c|c|c|c|c|c|c|c|}
\hline $\begin{array}{l}\text { Catégorie } \\
\text { de boucle }\end{array}$ & $\begin{array}{l}\text { No de } \\
\text { peau }\end{array}$ & $\begin{array}{l}\text { Largeur } \\
\text { de boucle }\end{array}$ & $\begin{array}{c}\text { Duvet } \\
\%\end{array}$ & $\begin{array}{l}\text { Catégorie } \\
\text { de boucle }\end{array}$ & $\begin{array}{l}\text { No de } \\
\text { peau }\end{array}$ & $\begin{array}{l}\text { Largeur } \\
\text { de boucle }\end{array}$ & $\begin{array}{c}\text { Duvêt } \\
\%\end{array}$ \\
\hline Moyenne.... & $\begin{array}{l}30 \\
3 \mathrm{I} \\
33 \\
34 \\
35\end{array}$ & $\begin{array}{l}7,0 \\
6,0 \\
5,0 \\
6,0 \\
6,0\end{array}$ & $\begin{array}{l}53 \\
47 \\
47 \\
37 \\
4 \mathrm{I}\end{array}$ & Grosse ... & $\begin{array}{l}40 \\
41 \\
42 \\
43 \\
44\end{array}$ & $\begin{array}{r}9,0 \\
10,0 \\
8,0 \\
10,0 \\
8,0\end{array}$ & $\begin{array}{l}45 \\
46 \\
47 \\
5^{2} \\
54\end{array}$ \\
\hline Tchakmaki. . & $\begin{array}{l}37 \\
38\end{array}$ & $\begin{array}{l}\text { I } 0,5 \\
\text { I } 1,5\end{array}$ & $\begin{array}{l}49 \\
60\end{array}$ & Petite .. & $\begin{array}{l}45 \\
47 \\
48 \\
49 \\
36\end{array}$ & $\begin{array}{l}4,0 \\
4,5 \\
4,0 \\
4,8 \\
4,0\end{array}$ & $\begin{array}{l}36 \\
46 \\
49 \\
55 \\
44\end{array}$ \\
\hline
\end{tabular}

En portant les pourcentages de duvet en ordonnée et la largeur des boucles correspondantes en abcisse, on obtient une dispersion irrégulière de points (fig. 57). Nous avons calculé le coefficient de corrélation qui 
est $r=+0,29$. Ce coefficient n'est pas significativement différent de zéro puisque la valeur $t=\mathrm{I}, 08$ pour un setil de probabilité de 0,05 est inférieure à celle qu'on devrait trouver $(t=2, \mathrm{I} 6 \mathrm{0})$ pour un nombre de degré de liberté égale à I3.

Par conséquent il n'existe pas de corrélation entre le pourcentage de duvet et la grosseur de la boucle.

En classant les peaux (sauf les tchakmakis) en bonnes et en médiocres d'après la qualité de leurs boucles nous avons trouvé les valeurs moyennes suivantes :

Peaux de bonne qualité : 46,3 p. Ioo de duvet.

Peaux de qualité médiocre : 46,8 p. Ioo de duvet.

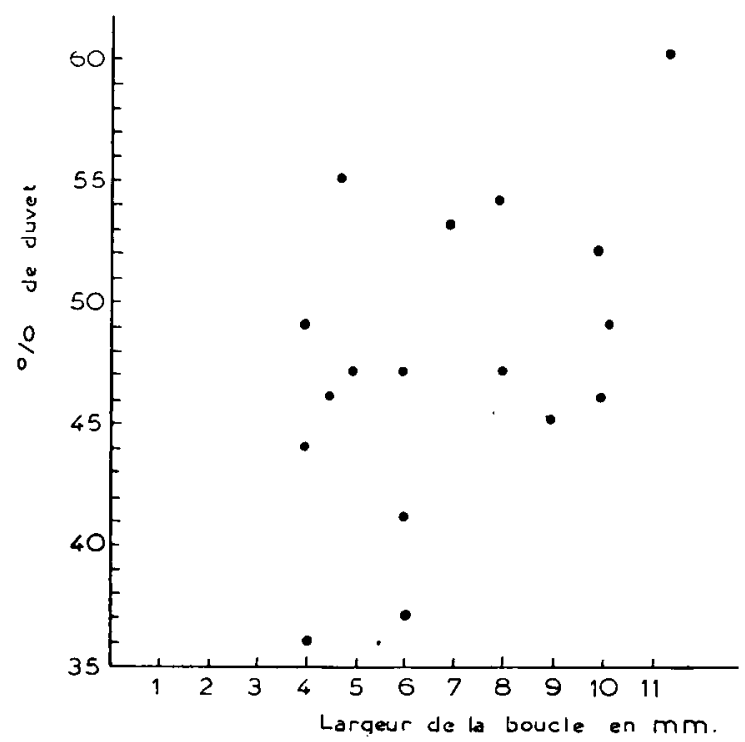

FIG. 57. - Relation entre le pourcentage de duvet et la largeur de la boucle.

Le pourcentage de duvet est aussi variable à l'intérieur d'un lot de peaux de bonne qualité qu'entre un bon et un mauvais lot. Cette variation est de 37 à $60 \mathrm{p}$. Ioo dans les peaux examinées.

On pourrait penser qu'un pourcentage élevé de duvet contribuerait activement au débouclage. En réalité il n'en est rien. Car les fibres secondaires (duvet) sont très courtes et très fines à la naissance de l'agneatu. En outre leur croissance de $2 \mathrm{~mm}$ par semaine rend peu vraisemblable que leur accroissement intervienne dans le débouclage.

En résumé il n'existe pas de corrélation entre la grosseur de la boucle et l'importance numérique relative du duvet. Cette dernière est en outre indépendante de la qualité de la boucle. 


\section{$2^{\circ}$ Diamètre des poils.}

Les mesures de diamètre des poils des peaux tannées ont été effectuées par la méthode de la W. I. R. A. Les inconvénients que nous avons décrits à propos de cette méthode sont en grande partie éliminés étant donné que les mesures portent sur des fibres primaires car ce sont les seules qui prennent part à la formation de la boucle. Le tableau XI montre les diamètres moyens calculés pour chaque peau.

I'ensemble des trois catégories de boucles ne forme pas une population homogène au point de vtre diamètre des poils. Le rapport de la variance entre colonne (catégorie de boucle) à la variance résiduelle $\frac{\mathrm{S}_{c}^{2}}{\mathrm{~S}_{t}^{2}}=5,63$ montre qu'il existe une probabilité très faible (inférieure à I p. Ioo) d'avoir un rapport de variances aussi élevé (seuil de $5 \mathrm{p}$. Ioo pour la valeur 3,37 et de I p. Ioo pour 5,63). Par conséquent le groupe des moyennes comporte des différences significatives.

\section{Tableau XI}

Relation entre la grosseur de la boucle et le diamètre moyen des poils (en $\mu$ ).

\begin{tabular}{|c|c|c|c|c|c|}
\hline $\begin{array}{l}\text { Catégorie de } \\
\text { boucle }\end{array}$ & $N^{\circ}$ de peau & $\begin{array}{c}\text { Diamètre moyen } \\
\text { des poils }\end{array}$ & $\begin{array}{c}\text { Catégorie de } \\
\text { boucle }\end{array}$ & No de peau & $\begin{array}{l}\text { Diamètre moyen } \\
\text { des poils }\end{array}$ \\
\hline & $\begin{array}{l}I \\
2 \\
3 \\
4 \\
5 \\
6\end{array}$ & $\begin{array}{l}56,20 \\
48,60 \\
47, \infty 0 \\
55,50 \\
5,3,86 \\
52,20\end{array}$ & Grosse & $\begin{array}{l}15 \\
16 \\
17 \\
18 \\
19\end{array}$ & $\begin{array}{l}45,82 \\
35,86 \\
34,72 \\
37,58 \\
42,44\end{array}$ \\
\hline Moyenne & $\begin{array}{r}7 \\
8 \\
9 \\
10 \\
\text { I } 1 \\
12 \\
13 \\
14\end{array}$ & $\begin{array}{l}57,06 \\
36,18 \\
48,36 \\
38,22 \\
51,10 \\
42,92 \\
49,94 \\
46,24\end{array}$ & Petite & $\begin{array}{l}20 \\
21 \\
22 \\
23 \\
24 \\
25 \\
26 \\
27 \\
28 \\
29\end{array}$ & $\begin{array}{l}48,78 \\
45,26 \\
43,90 \\
53,58 \\
44,28 \\
41,08 \\
48,16 \\
54,80 \\
45,60 \\
46,00\end{array}$ \\
\hline
\end{tabular}

En comparant les moyennes des diamètres des poils des trois catégories de boucles deux à deux, on constate que les grosses boucles ont des poils de diamètre significativement plus faible que ceux des boucles moyennes et petites. Par contre, les diamètres des poils des boucles moyennes et petites ne diffèrent pas significativement.

Par conséquent il semble $y$ avoir une relation inverse entre le diamètre moyen des poils et la taille des boucles.

Pour la qualité des boucles, nous avons trouvé une différence de diamètre moyen entre la bonne et la mauvaise qualité pour les boucles moyennes mais il ne semble pas qu'il y ait d'association entre le dia- 
mètre moyen des poils et la qualité des petites boucles, comme le montrent les chiffres suivants :

Diamètre moyen :

$5 I, 84 \mu$ pour la boucle moyenne de bonne qualité.

$43,35 \mu$ pour la boucle moyenne de mauvaise qualité.

$47, I 6 \mu$ pour la petite boucle de bonne qualité.

$47, \mathrm{I} 3 \mu$ pour la petite boucle de mauvaise qualité.

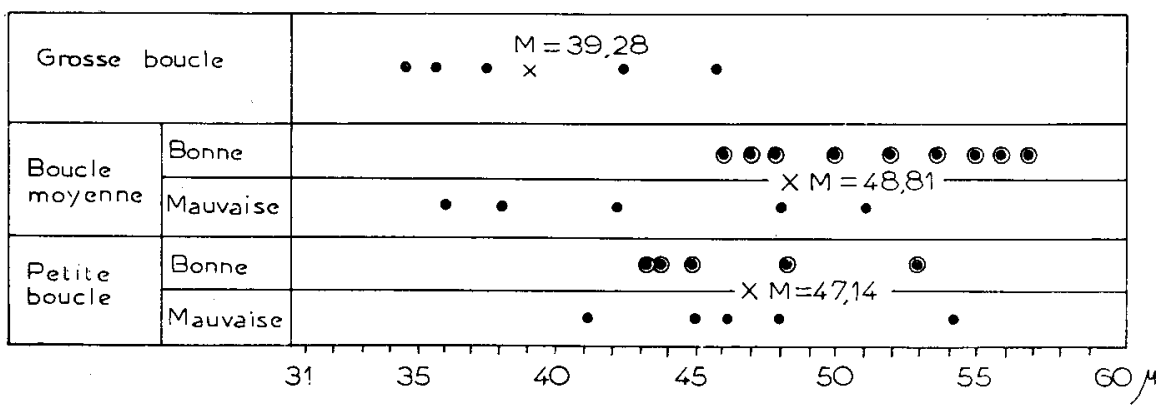

FIG. 58. - Relation entre le diamètre moyen des poils et la grosseur de la boucle.

Quant aux grosses boucles, il est assez difficile de faire des rapprochements, étant donné qu'elles sont toutes de qualité moyenne ou médiocre. La figure 58 montre la dispersion des diamètres moyens des poils pour les trois catégories de grosseur des boucles.

\section{$3^{0}$ Présence de la moelle.}

La proportion des fibres médullées est très variable d'un individu à l'autre, comme le montre le tableau XII.

Nous avons groupé les pourcentages des fibres médullées selon les 4 catégories de boucles (petite, moyenne, grosse et la boucle composée du type Tchakmaki) (fig. 59).

Les pourcentages moyens sont les suivants :

Petite boucle : 6,07 p. Ioo de fibres médullées.

Boucle moyenne : I7,00 p. Ioo de fibres médullées.

Grosse boucle : 17,25 p. Ioo de fibres médullées.

Tchakmaki : 35,00 p. Ioo de fibres médullées (2 peaux).

On constate ainsi que la proportion des fibres médullées est très faible ( $6 \mathrm{p}$. Ioo en moyenne) pour les petites boucles et très variable, mais en moyenne trois fois plus importante, pour les boucles moyennes, grosses et Tchakmaki.

Sur la figure 59 on remarque également que les points correspondants aux peaux de bonne qualité (point entouré d'un cercle) et aux peaux médiocres (point seu1) étant dispersés de façon irrégulière, on ne peut y 
TABLEAAU XII

Relation entre la grosseur de la boucle et le pourcentage des fibres médullées.

\begin{tabular}{|c|c|c|c|c|c|}
\hline Catégorie de boucle & $\begin{array}{c}\text { No de } \\
\text { peau }\end{array}$ & $\begin{array}{l}\% \text { de fibres } \\
\text { médullées }\end{array}$ & Catégories de boucle & $\begin{array}{l}\text { No de } \\
\text { peau }\end{array}$ & $\begin{array}{l}\% \text { de fibres } \\
\text { médullées }\end{array}$ \\
\hline \multirow[t]{2}{*}{ Moyenne } & $\begin{array}{l}\text { I } \\
2 \\
3 \\
4 \\
5 \\
6 \\
7 \\
8 \\
9\end{array}$ & $\begin{array}{r}\text { I3 } \\
30 \\
\text { I5 } \\
36 \\
20 \\
6 \\
0 \\
0 \\
\text { I3 }\end{array}$ & Grosse & $\begin{array}{l}15 \\
16 \\
17 \\
18 \\
40 \\
41 \\
43 \\
44\end{array}$ & $\begin{array}{r}7 \\
5 \\
9 \\
12 \\
30 \\
24 \\
23 \\
28\end{array}$ \\
\hline & $\begin{array}{l}\text { I I } \\
\text { I } 2 \\
\text { I3 } \\
\text { I4 } \\
30 \\
3 \text { I } \\
33 \\
34 \\
35\end{array}$ & $\begin{array}{r}24 \\
17 \\
0 \\
0 \\
0 \\
30 \\
35 \\
1 \\
40 \\
26\end{array}$ & \multirow[t]{2}{*}{ Petite } & $\begin{array}{l}20 \\
22 \\
23 \\
24 \\
25 \\
26 \\
28 \\
29 \\
45 \\
47\end{array}$ & $\begin{array}{c}\text { 10 } \\
\text { I3 } \\
0 \\
0 \\
0 \\
0 \\
0 \\
7 \\
6 \\
0 \\
6\end{array}$ \\
\hline Tchakmaki & $\begin{array}{l}38 \\
39\end{array}$ & $\begin{array}{l}28 \\
42\end{array}$ & & $\begin{array}{l}48 \\
49 \\
3^{6}\end{array}$ & $\begin{array}{r}0 \\
31 \\
6\end{array}$ \\
\hline
\end{tabular}

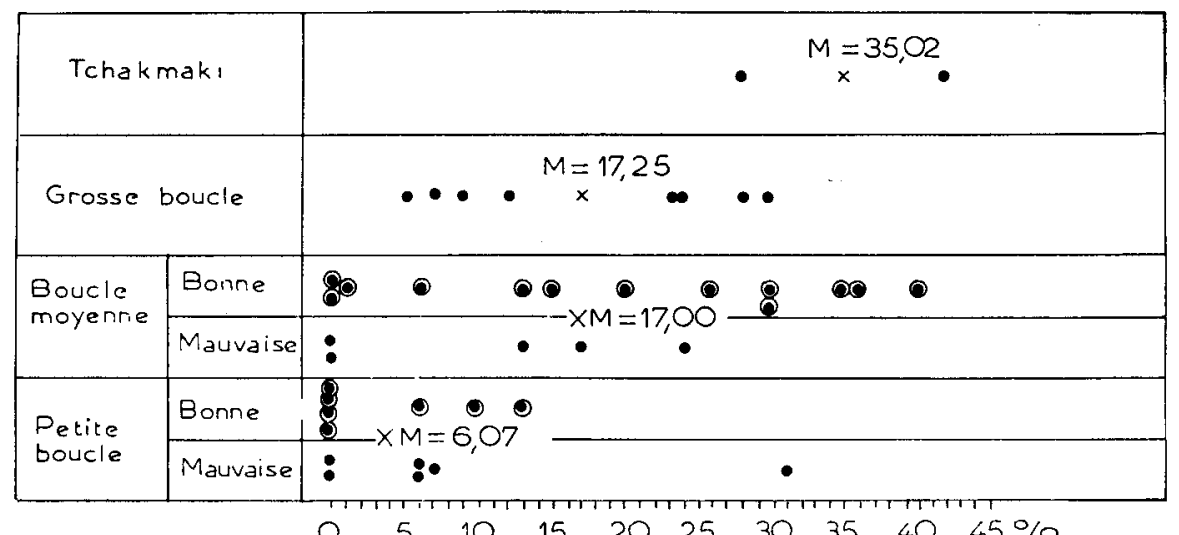

FIG. 59. - Relation entre la grosseur de la boucle et le pourcentage des fibres médullées.

constater une association entre le pourcentage des fibres médullées et la qualité de la fourrure.

En résumé les petites boucles comprennent un pourcentage peu élevé de fibres médullées tandis que ce pourcentage est très différent quoique variable dans les autres boucles moyennes, grosses et Tchakmaki.

La qualité de la boucle est indépendante du pourcentage de fibres médullées. 


\section{$4^{0}$ Forme de la section des poils.}

Nous avons vu à propos de l'incurvation des fibres que la forme de leur section varie d'un animal à l'autre et chez le même individu, d'un poil à l'autre. Cela explique combien il est difficile de classer les peaux suivant les différentes formes de la section de leurs poils.

\section{$5^{\circ}$ Longueur des fibres primaires (poils).}

Les mesures de longueur des poils ont été effectuées sur I7 peaux réparties comme suit :

5 peaux à petites boucles,

5 peaux à grosses boucles,

5 peaux à boucles moyennes,

2 peaux à boucles composées très larges (Tchakmaki).

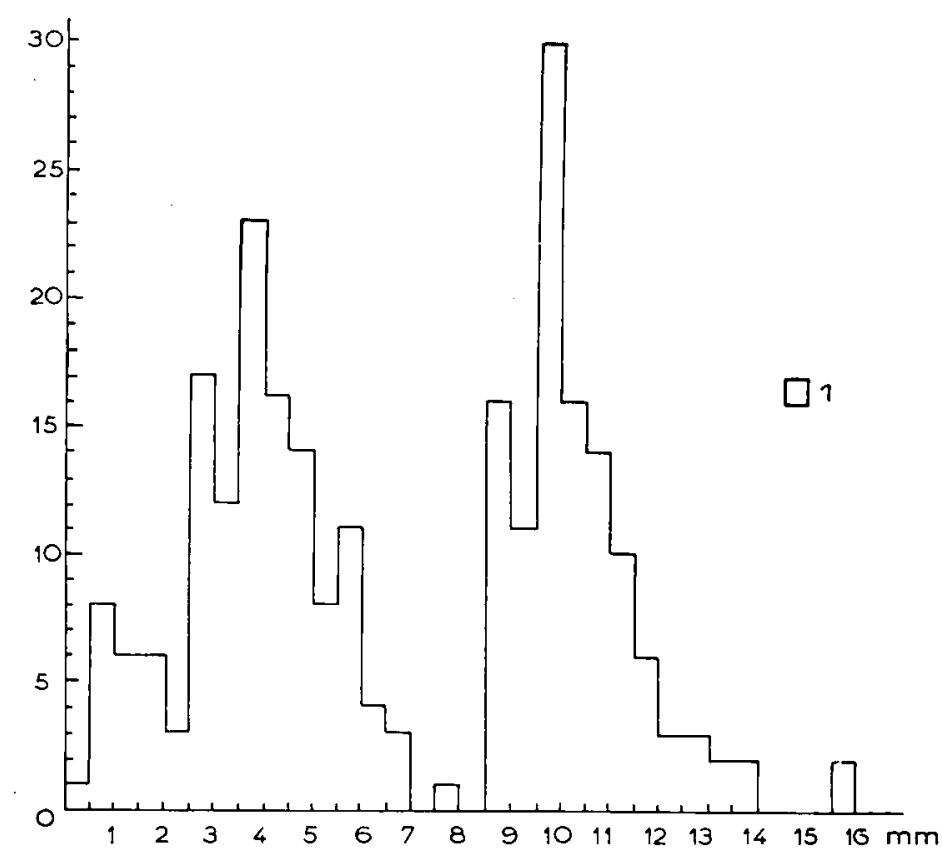

FIG. 60. - Ilistogramme de la longueur des fibres d'une boucle moyenne chez l'agneau Karakul à la naissance. Remarquer la distinction nette des deux populations : duvet et poils.

Sur chaque peau ont été choisies une ou deux boucles caractéristiques de la catégorie envisagée. 3583 mesures ont été effectuées formant I7 histogrammes. La figure 60 montre l'un d'eux représentant 250 mesures sur une boucle moyenne.

Les histogrammes sont caractéristiques et marquent nettement la distinction entre les poils (à droite) et le duvet (à gauche). Dans l'ensem- 
ble la courbe de fréquence du duvet a une allure régulière alors que celle des poils a une allure dissymétrique, le sommet étant nettement situé sur la gauche.

Les moyennes des longueurs des poils pour chaque histogramme ainsi que les grosseurs des boucles correspondantes figurent sur le tableau XIII.

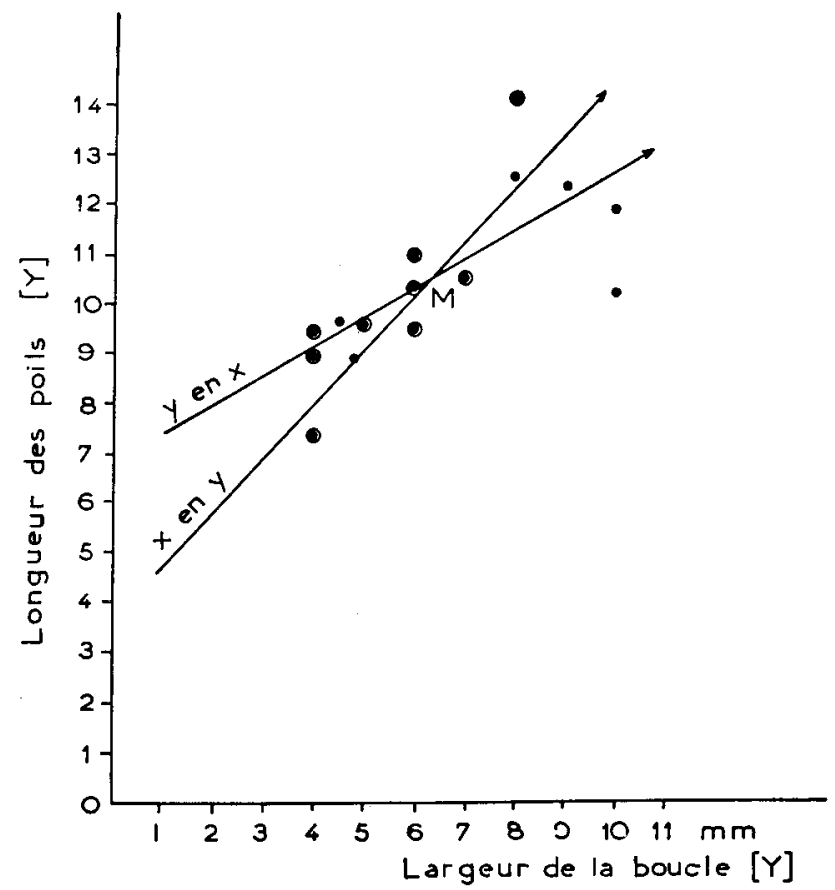

Fig. 6r. - Corrélation entre la largeur de la boucle et la longueur des poils $; \mathrm{r}=+0,74 ; \mathrm{b} y, x=0,60$;

$\mathrm{b}_{x}, y=0,9 \mathrm{I}$.

Les points cerclés représentent les fourrures de bonne qualité.

En groupant les longueurs par catégorie de boucle on trouve les moyennes suivantes :

$8,93 \mathrm{~mm}$ pour les petites boucles,

I0,24 $\mathrm{mm}$ pour les boucles moyennes,

$\mathrm{r} 2,36 \mathrm{~mm}$ pour les grosses boucles.

Pour le type Tchakmaki la longueur moyenne des poils est égale à 9,3 $\mathrm{Imm}$. En réalité ce n'est pas une boucle proprement dite mais une boucle composée (voir annexe), aussi nous n'en avons pas tenu compte.

L'analyse statistique montre une probabilité élevée pour qu'une corrélation positive existe entre la longueur des poils et la grosseur des boucles : $r=+0,74$ qui est très significativement différent de zéro $(t=3,96)$.

La corrélation entre les deux caractères est représentée par les droites de régression $x$ en $y$ (pente $=0,9$ I et y en $x$ (pente $=0,6$ ) (fig. 6I). 


\section{TABLEAU XIII}

Relation entre la grosseur de la boucle (en $\mathrm{mm}$ ) et la longueur moyenne des poils (en mm).

\begin{tabular}{|c|c|c|c|}
\hline Catégorie de boucle & No de peau & Largeur de boucle & $\begin{array}{l}\text { Longueur moyenne } \\
\text { des poils }\end{array}$ \\
\hline Moyenne & $\begin{array}{l}30 \\
31 \\
33 \\
34 \\
35\end{array}$ & $\begin{array}{l}7,0 \\
6,0 \\
5,0 \\
6,0 \\
6,0\end{array}$ & $\begin{array}{r}\text { I0,6 I } \\
\text { I0,40 } \\
9,66 \\
\text { I I, } 00 \\
9,53\end{array}$ \\
\hline Tchakmaki & $\begin{array}{l}37 \\
38\end{array}$ & $\begin{array}{l}\text { IO,2 } \\
\text { II,2 }\end{array}$ & $\begin{array}{r}8,24 \\
10,40\end{array}$ \\
\hline Grosse & $\begin{array}{l}40 \\
4 \mathrm{I} \\
42 \\
43 \\
44\end{array}$ & $\begin{array}{r}9,0 \\
10,0 \\
8,0 \\
10,0 \\
8,0\end{array}$ & $\begin{array}{l}\text { I 2,5 I } \\
\text { I 2,06 } \\
\text { I 2,66 } \\
\text { I } 0,37 \\
\text { I 4,20 }\end{array}$ \\
\hline Petite & $\begin{array}{l}45 \\
47 \\
48 \\
49 \\
36\end{array}$ & $\begin{array}{l}4,0 \\
4,5 \\
4,0 \\
4,8 \\
4,0\end{array}$ & $\begin{array}{l}9,50 \\
9,73 \\
7,39 \\
8,96 \\
9,08\end{array}$ \\
\hline
\end{tabular}

L_es grosses boucles étant très souvent de qualité médiocre et formées des poils les plus longs, il existe évidemment une association entre la mauvaise qualité de la boucle et la grande longueur des poils. Par contre, chez les boucles moyennes et petites on ne peut établir une association nette entre la qualité de la boucle et la longueur des poils.

\section{$6^{\circ}$ Épaisseur de la peau.}

Les mesures de l'épaisseur de la peau ont été effectuées au niveau du dos mais non sur la ligne dorsale, sur 20 peaux tannées et apprêtées d'origine afghane. La tableau XIV représente les valeurs obtenues et classées par catégorie de boucles.

Les épaisseurs moyennes des peaux sont les suivantes :

I,02 $\mathrm{mm}$ pour les peaux à petites boucles.

I,I $9 \mathrm{~mm}$ pour les peaux à boucles moyennes.

r,33 mm pour les peaux Tchakmaki.

I,35 $\mathrm{mm}$ pour les peaux à grosses boucles.

La figure 62 montre la variation de l'épaisseur de la peau en fonction de la largeur (ou grosseur) des boucles.

Le calcul montre qu'il y a une probabilité élevée pour qu'une corrélation positive existe entre ces deux caractères. Le coefficient de corrélation $r=+0,59$ est significativement différent de zéro $(t=2,83$ pour I5 degrés de liberté). Cette corrélation est représentée sur la figure 62 


\section{TABLEAU XIV}

L'épaisseur de la peau et la densité pileuse en fonction de la grosseur de la boucle.

\begin{tabular}{|c|c|c|c|c|}
\hline $\begin{array}{l}\text { Catégrorie } \\
\text { de boucle }\end{array}$ & No de peau & $\begin{array}{l}\text { Largeur } \\
\text { de boucle }\end{array}$ & $\begin{array}{l}\text { Élaisseur } \\
\text { de la peau }\end{array}$ & Densité pileuse \\
\hline Yoyennes & $\begin{array}{l}30 \\
3 \mathrm{I} \\
32 \\
33 \\
34 \\
35\end{array}$ & $\begin{array}{l}7,0 \\
6,0 \\
5,5 \\
5,0 \\
6,0 \\
6,0\end{array}$ & $\begin{array}{l}1,20 \\
\mathrm{I}, 32 \\
\mathrm{I}, \mathrm{1} 2 \\
\mathrm{1}, 05 \\
\mathrm{I}, 33 \\
\mathrm{I}, 15\end{array}$ & $\begin{array}{l}42,4 \\
43,4 \\
55,0 \\
46,0 \\
50,0 \\
62,0\end{array}$ \\
\hline Tchakmaki & $\begin{array}{l}37 \\
38 \\
39\end{array}$ & $\begin{array}{l}\text { I } 0,2 \\
\text { I I, } 5 \\
\text { I } 0,3\end{array}$ & $\begin{array}{l}1,42 \\
1,25 \\
1,34\end{array}$ & $\begin{array}{l}56,0 \\
47,4 \\
55, \circ\end{array}$ \\
\hline Grosses & $\begin{array}{l}40 \\
4 \mathrm{I} \\
42 \\
43 \\
44\end{array}$ & $\begin{array}{r}9,0 \\
10,0 \\
8,0 \\
10,0 \\
8,0\end{array}$ & $\begin{array}{l}1,45 \\
1,27 \\
1,05 \\
1,38 \\
1,60\end{array}$ & $\begin{array}{l}46,0 \\
40,0 \\
48,0 \\
46,0 \\
46,0\end{array}$ \\
\hline Petites & $\begin{array}{l}45 \\
46 \\
47 \\
48 \\
49 \\
3^{6}\end{array}$ & $\begin{array}{l}4,0 \\
3,7 \\
4,5 \\
4,0 \\
4,8 \\
4,0\end{array}$ & $\begin{array}{l}\mathrm{I}, 28 \\
\mathrm{I}, 20 \\
0,77 \\
0,93 \\
\mathrm{I}, \mathrm{I} \\
0,75\end{array}$ & $\begin{array}{l}47,0 \\
45,4 \\
46,4 \\
46,6 \\
48,0 \\
56,0\end{array}$ \\
\hline
\end{tabular}

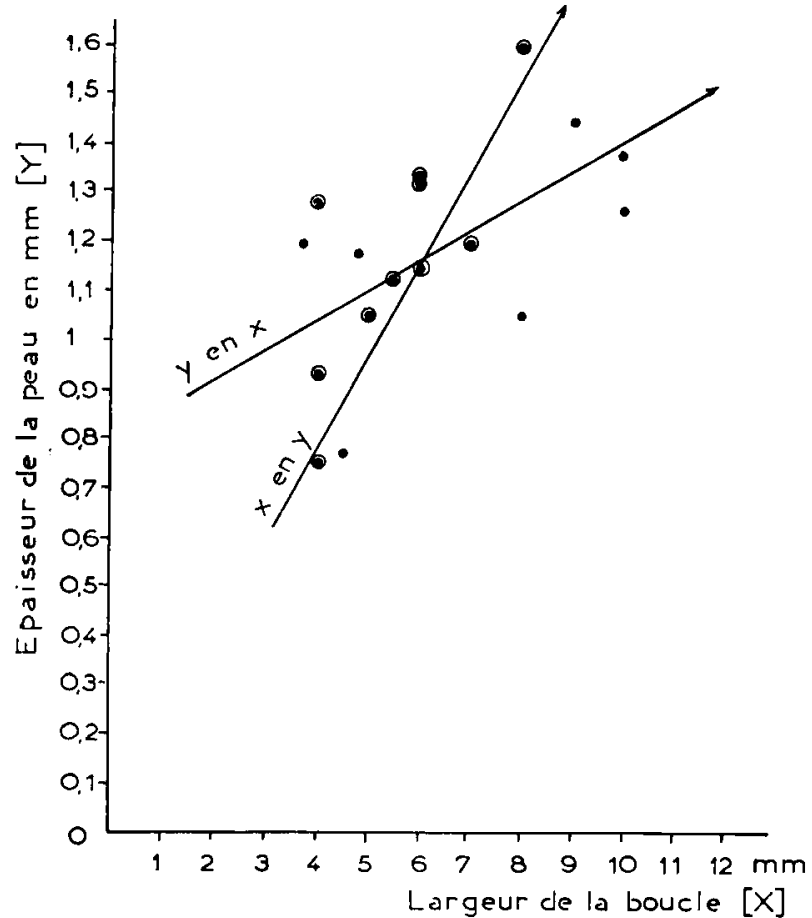

Iis. 62. - Corrélation entre lat largeur de la boucle et l'épaisseur de la peau $; \mathrm{r}=+0,59 ; \mathrm{b}_{y, x}=0,0,62$; $\mathrm{b}_{x, y}=5,55$. 
par les deux droites de régression de $x$ en $y$ (pente $=5,55$ ) et de $y$ en $x$ (pente $=0,062$ ).

La même figure montre la répartition des points représentant les peaux de bonne qualité (points entourés d'un cercle) et de ceux correspondant aux boucles de qualité médiocre (points seuls). Ces répartitions ne semblent pas être en relation directe avec l'épaisseur de la peau.

Nous venons de démontrer que la grosseur de la boucle est en corrélation positive avec la longueur des poils et avec l'épaisseur de la peaut. E.t, comme on pouvait s'y attendre, la valeur de $r=+0,75$ montre

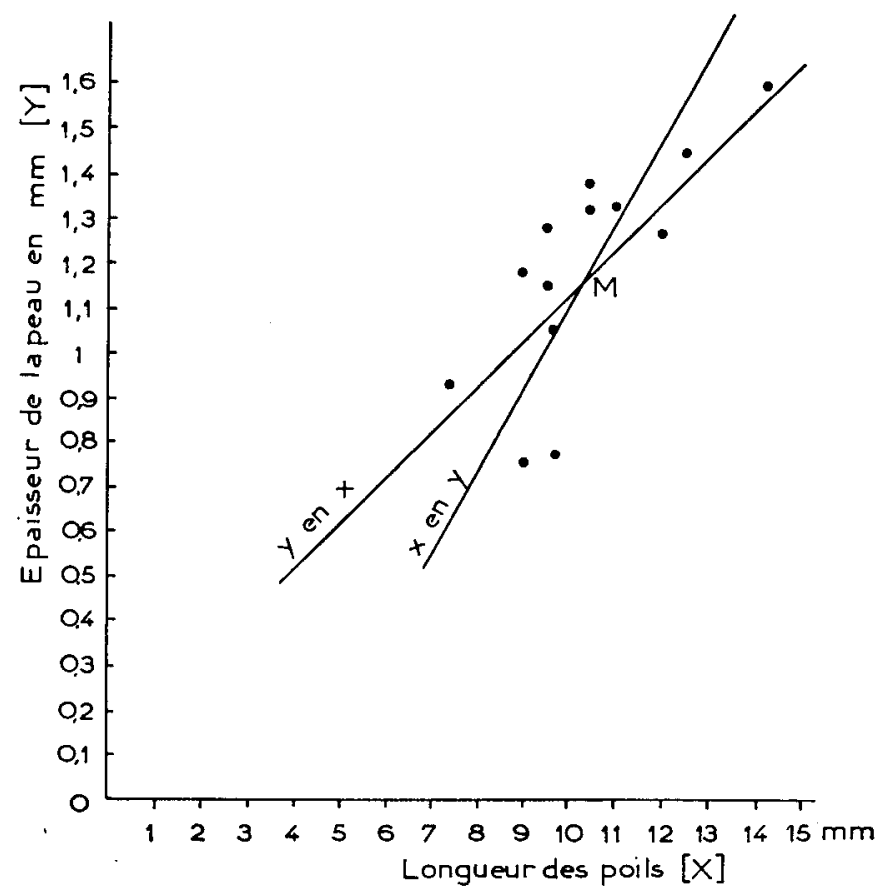

FiG. 63. - Corrélation entre la longueur des poils et l'épaisseur de la peau $; \mathrm{r}=+0,75 ; \mathrm{b}_{y, x}=0, \mathrm{I}_{4}$;

$$
\mathrm{b}_{x, y}=5,49 \text {. }
$$

une probabilité élevée pour qu'il existe une corrélation positive entre ces deux derniers caractères $(t=4, \mathrm{II})$ (fig. 63).

\section{$7^{\circ}$ Densité pileuse.}

La détermination de la densité pileuse sur les peaux tannées est basée aussi bien sur le duvet (fibres secondaires) que sur les poils proprement dits (fibres primaires).

Les valeurs obtenues à la suite du comptage sous la loupe binoculaire sur les peaux tannées sont très différentes de celles obtenues par le procédé histologique sur les échantillons frais, qui est plus complet. 
Néanmoins, la loupe permet de comparer le nombre de fibres par unité de surface pour les peaux tannées.

Les valeurs obtenues (tableau XIV) ont été comparées avec la catégorie et la classe des peaux. Les densités moyennes sont les suivantes :

Petites boucles : $48,23 \mathrm{~mm}^{2}$.

Boucles moyennes : 49,80 $\mathrm{mm}^{2}$.

Grosses boucles : $45,20 \mathrm{~mm}^{2}$.

Tchakmaki : $52,80 \mathrm{~mm}^{2}$.

Ces moyennes sont aussi variables entre les différentes catégories qu'à l'intérieur de celles-ci. Il en résulte donc que la densité pileuse n'est en rapport ni avec la grosseur de la boucle ni avec la qualité de celle-ci et de la peau.

La densité pileuse a été comparée également avec l'épaisseur des peaux. Aucun résultat positif n'a été obtenu.

\section{DISCUSSION}

Nous venons de voir que certaines caractéristiques de la peau et des fibres peuvent être en association avec la qualité et la grosseur des boucles, tandis que pour d'autres caractéristiques telles que le pourcentage de duvet et la forme de la section, cette relation n'existe pas. Or ces résultats ne sont pas toujours en accord avec les opinions des auteurs qui se sont occupés de cette question. Il est donc intéressant de savoir d'où vient cette divergence.

Nous avons vu que NicOv (I930) trouve, pour les boucles médiocres, un pourcentage de duvet plus important que pour les bonnes boucles. Or nous savons que les boucles médiocres sont en général de grande taille et constituées par des fibres plus longues et moins serrées contre la surface de la peau.

En prélevant ses échantillons à l'aide des ciseaux, Nicov a pu prélever la presque totalité du duvet pour les grosses boucles (médiocres) alors que pour les bonnes boucles dont les fibres sont plus courtes et plus serrées contre la surface de la peau, une certaine proportion de duvet lui a échappé.

Pour le diamètre des fibres, TäNzER (1928) ne trouve pas de relation avec la grosseur de la boucle. Mais comme nous avons vu, au chapitre $I$, le diagramme publié par TÄNzER est très différent de celui que nous avons tracé pour le diamètre des fibres. En effet le diagramme de TäNZER comporte un seul sommet alors que-nous avons trouvé deux ou trois sommets. Cette divergence provient de la méthode utilisée, ce qui explique l'opinion de TäNzER selon laquelle il n'y a pas de différence de diamètre des poils entre les différentes grosseurs de boucle. Par contre nos résultats montrant la relation entre un diamètre fort des poils et une taille petite de la boucle confirme ceux de LANGLET (I936). 
TäNZER affirme d'autre part que la présence du canal médullaire dans le poil est en général nuisible à la formation de la boucle. Il est vrai que le rayon de courbure est en général proportionnel à la quantité de moelle dans les poils, mais la qualité de la boucle est indépendante de la présence de la moelle si on considère les boucles de la même grosseur.

L'opinion de LOSSEN (I92I) qui reconnait une relation entre la forme en " triangle isocèle " de la section transversale du poil et la bonne qualité de la boucle, ne pourrait être prise en considération, étant donné le polymorphisme de cette section, comme nous l'avons montré précedemment, ce qui confirme le point de vue de TänzER (I928) à ce sujet.

\section{TABLEAU XV}

Opinions des auteurs sur la relation des caractéristiques de la peau et $d u$ poil avec la grosseur et la qualité de la boucle.

\begin{tabular}{|c|c|c|c|}
\hline Caractéristiques & & Grosseur de la boucle & Qualité de la boucle \\
\hline Pourcentage de duvet & $\begin{array}{c}\text { Relation } \\
\text { Pas de relation }\end{array}$ & NACER (I956) & $\begin{array}{l}\text { Nicov (1930) } \\
\text { NACER (1956) }\end{array}$ \\
\hline Diamètre des poils & $\begin{array}{c}\text { Relation } \\
\text { Pas de relation }\end{array}$ & $\begin{array}{l}\text { Nacer }(\text { I956) } \\
\text { Tanzer (r928) }\end{array}$ & $\begin{array}{c}\text { Nacer ( }\left(195^{6}\right) \\
\text { TANZER (1928) } \\
\text { Nicov (r930) }\end{array}$ \\
\hline $\begin{array}{c}\text { Présence } \\
\text { du canal médulaire }\end{array}$ & $\begin{array}{c}\text { Relation } \\
\text { Pas de relation }\end{array}$ & Nacer $\left(195^{6}\right)$ & $\begin{array}{l}\text { TANZER (1928) } \\
\text { NACER (1956) }\end{array}$ \\
\hline $\begin{array}{c}\text { Forme de la section droite } \\
\text { des fibres }\end{array}$ & $\begin{array}{c}\text { Relation } \\
\text { Pas de relation }\end{array}$ & NACER (1956) & $\begin{array}{l}\text { LOSSEN (1921) } \\
\text { TANZER (1928) } \\
\text { NACER }\left(195^{6}\right)\end{array}$ \\
\hline Longueur des poils & $\begin{array}{c}\text { Relation } \\
\text { Pas de relation }\end{array}$ & NACER (1956) & $\begin{array}{l}\text { NiCOV (1930) } \\
\text { LANGLET (1936) } \\
\text { NACER (1956) }\end{array}$ \\
\hline Épaisseur de la peau & $\begin{array}{c}\text { Relation } \\
\text { Pas de relation }\end{array}$ & NACER (1956) & $\begin{array}{c}\text { LANGLET (I936) } \\
\text { HARDY } \\
\text { \& SIMMONS ( } 1943) \\
\left.\text { NACER ( } 95^{6}\right)\end{array}$ \\
\hline Densité pileuse & $\begin{array}{c}\text { Relation } \\
\text { Pas de relation }\end{array}$ & Nacer $\left(195^{6}\right)$ & $\begin{array}{l}\text { TANZER (I } 928) \\
\text { NACER (1956) }\end{array}$ \\
\hline
\end{tabular}

En ce qui concerne la longueur des poils, il existe une corrélation positive entre elle et la grosseur de la boucle. Cette relation est si étroite que même les méthodes moins précises l'ont mise en évidence. C'est le cas notamment de la méthode des pinces utilisée par Nrcov (I930).

Pour l'épaisseur de la peau, ADAMETz I9I4 a montré que l'épaisseur de la peau de l'oreille est proportionnelle à l'épaisseur de la peau sur le reste du corps. Cette indication a été utilisée par HaRdy et Stmmons 
(I943) qui ont mesuré l'épaisseur de la peau au niveau de l'oreille sur des agneaux Karakul vivants, en pinçant la peau et en la mesurant au Palmer sur une double épaisseur. Ils ont trouvé ainsi qu'une peau mince produit des boucles de qualité supérieure, ce qui est confirmé par I,ANGLET (I936) et par nous-mêmes.

I a densité pileuse sans rapport avec la qualité des boucles, comme l'a reconnu TÄNzER (I928) est également indépendante de la grosseur de la boucle.

Mais, tandis que les travaux anciens (tableau XV) furent le plus souvent basés sur des observations imprécises nous avons trouvé des corrélations indiscutables entre certaines caractéristiques de la peau et des poils. Ainsi nous avons montré que l'épaisseur de la peau est en corrélation positive d'une part avec la grosseur de la boucle et d'autre part avec la longueur des poils. Nous avons également mis en évidence la corrélation positive qui existe entre la grosseur de la boucle et la longueur des poils.

\section{CONCLUSION}

I.e matériel que nous avions à notre disposition ne nous a pas permis d'établir des corrélations entre d'une part, la grosseur et la qualité de la boucle et d'autre part la forme de la section des fibres, le pourcentage de duvet et le pourcentage de poils médullés. Toutefois ces derniers sont rares dans les petites boucles.

Par contre nous avons montré que les poils des petites boucles avaient un diamètre moyen significativement plus fort $(47$, I $4 \mu)$ que celui des grosses boucles $(39,28 \mu)$ qui sont souvent de qualité médiocre.

Nous avons également montré qu'il existait une corrélation positive entre l'épaisseur de la peau et la grosseur de la boucle et la longueur des poils.

Ainsi aux peaux minces sont généralement associés des poils courts et de fort diamètre correspondant à des fourrures de bonne qualité, par contre les peaux épaisses présentent le plus souvent des poils plus longs et plus fins correspondant à des boucles plus grosses et à des qualités médiocres de la fourrure.

Comme l'épaisseur de la peau est en relation directe avec son poids et que la légèreté est en elle-même une qualité appréciable, à ce double titre les animaux à peau mince sont les plus intéressants.

(A suivre). 